\title{
Geodesic Walks in Polytopes
}

\author{
Yin Tat Lee*, Santosh S. Vempala ${ }^{\dagger}$
}

June 2, 2017

\begin{abstract}
We introduce the geodesic walk for sampling Riemannian manifolds and apply it to the problem of generating uniform random points from the interior of polytopes in $\mathbb{R}^{n}$ specified by $m$ inequalities. The walk is a discrete-time simulation of a stochastic differential equation (SDE) on the Riemannian manifold equipped with the metric induced by the Hessian of a convex function; each step is the solution of an ordinary differential equation (ODE). The resulting sampling algorithm for polytopes mixes in $O^{*}\left(m n^{\frac{3}{4}}\right)$ steps. This is the first walk that breaks the quadratic barrier for mixing in high dimension, improving on the previous best bound of $O^{*}(m n)$ by Kannan and Narayanan for the Dikin walk. We also show that each step of the geodesic walk (solving an ODE) can be implemented efficiently, thus improving the time complexity for sampling polytopes. Our analysis of the geodesic walk for general Hessian manifolds does not assume positive curvature and might be of independent interest.
\end{abstract}

\section{Contents}

1 Introduction $\quad 3$

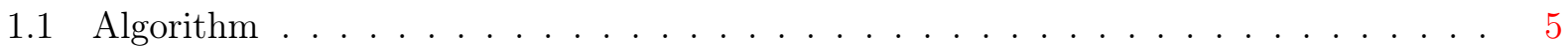

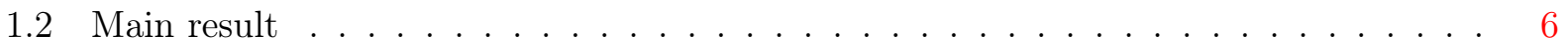

1.3 Discussion and open problems . . . . . . . . . . . . . . . . 7

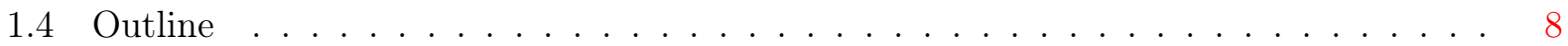

2 Background and notation $\quad 9$

2.1 Basic Definitions of Riemannian geometry . . . . . . . . . . . . . . . . 9

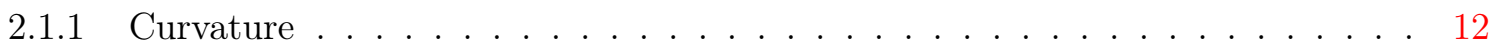

2.1 .2 Jacobi field . . . . . . . . . . . . . . . . . . . . . . . . . . . . . . . . . . . . . . .

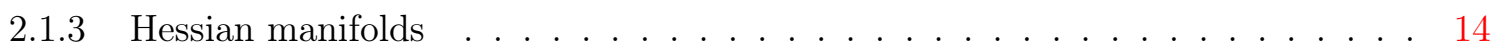

2.1.4 Normal coordinates . . . . . . . . . . . . . . . . . . . . 15

2.2 Stochastic calculus . . . . . . . . . . . . . . . . . . . 15

2.2.1 Derivation of the Geodesic walk . . . . . . . . . . . . . . . 16

2.3 Complex analysis . . . . . . . . . . . . . . . . . . . . 18

3 Convergence of the Geodesic Walk 20

3.1 Hessian parameters . . . . . . . . . . . . . . . . . 20

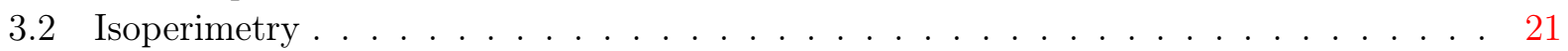

3.3 1-step distribution . . . . . . . . . . . . . . . . . . . . . . . 22

*Microsoft Research and University of Washington, yintat@uw.edu

${ }^{\dagger}$ Georgia Tech, vempala@gatech.edu 
3.4 Conductance and mixing time . . . . . . . . . . . . . . . . . . 24

3.5 Warm-up: Interval and hypercube . . . . . . . . . . . . . . . 25

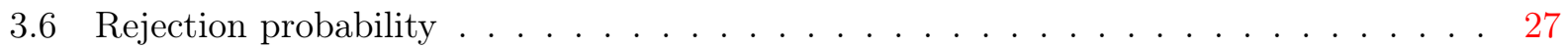

3.6 .1 Trapezoidal rule . . . . . . . . . . . . . . . . . . . . 28

3.6.2 Smoothness of exponential map . . . . . . . . . . . . . . . . . . . . . . . . . . . . . . . . . . . . . . . . . . . . .

3.7 Smoothness of one-step distributions . . . . . . . . . . . . . . . . . . . . . 32

3.8 Approximate solution of Jacobi field equations _ . . . . . . . . . . . . . 37

3.9 Almost one-to-one correspondence of geodesics . . . . . . . . . . . . . . 39

3.10 Implementation . . . . . . . . . . . . . . . . . . . . . . . . 42

4 Logarithmic barrier $\quad 43$

4.1 Riemannian geometry on $M_{L}\left(G_{2}\right) \ldots \ldots \ldots \ldots \ldots$

4.2 Geodesic walk on $M_{L} \ldots \ldots \ldots \ldots \ldots \ldots \ldots$

4.3 Randomness and stability of the geodesic $\left(V_{0}\right) \ldots \ldots \ldots \ldots$

4.4 Stability of Drift $\left(D_{0}, D_{1}\right.$ and $\left.D_{2}\right) \ldots \ldots \ldots \ldots \ldots \ldots \ldots$

4.5 Smoothness of the metric $\left(G_{1}\right) \ldots \ldots \ldots \ldots \ldots$

4.6 Stability of Curvatures $\left(R_{1}\right.$ and $\left.R_{2}\right) \ldots \ldots \ldots \ldots \ldots$

4.7 Stability of $L_{4}+L_{\infty}$ norm $\left(V_{1}\right) \ldots \ldots \ldots \ldots \ldots \ldots$

4.8 Mixing Time . . . . . . . . . . . . . . . . . . . 59

5 Collocation Method for ODE $\quad 61$

5.1 First Order ODE . . . . . . . . . . . . . . . . . . . . . . . 61

5.2 Second Order ODE . . . . . . . . . . . . . . . . . . 67

5.3 Example: Discretization of Physarum dynamics . . . . . . . . . . . . . . 68

5.4 Derivative Estimations . . . . . . . . . . . . . . . . . . . 71

5.5 Explicit Function . . . . . . . . . . . . . . . . . . . . 71

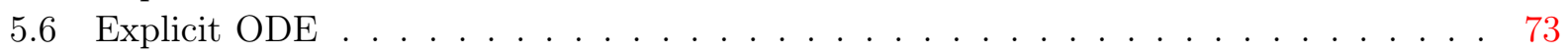

6 Implementation of Geodesic walk for log barrier $\quad 76$

6.1 Complex Analyticity of geodesics, parallel transport and Jacobi fields . . . . . . . 76

6.1 .1 Geodesic . . . . . . . . . . . . . . . . . 76

6.1 .2 Parallel Transport . . . . . . . . . . . . . . . . . . . 78

6.1 .3 Jacobi field . . . . . . . . . . . . . . . . . . . . . 80

6.2 Computing Geodesic Equation . . . . . . . . . . . . . . . . . 82

6.3 Computing Parallel Transport . . . . . . . . . . . . . . . . . . . . . 84

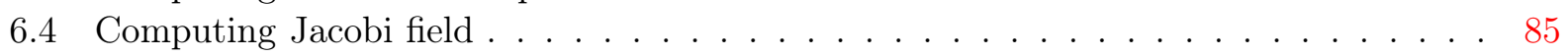

6.5 Computing Geodesic Walk . . . . . . . . . . . . . . . . . 86

$\begin{array}{lr}\text { A Additional proofs } & 89\end{array}$

Acknowledgement. The authors thank Sébastien Bubeck, Ben Cousins, Ton Dieker, Chit Yu Ng, Sushant Sachdeva, Nisheeth K. Vishnoi and Chi Ho Yuen for helpful discussions. Part of this work was done while visiting the Simons Institute for the Theory of Computing, UC Berkeley. The authors thank Yan Kit Chim for making the illustrations. 


\section{Introduction}

Sampling a high-dimensional polytope is a fundamental algorithmic problem with many applications. The problem can be solved in randomized polynomial time. Progress on the more general problem of sampling a convex body given by a membership oracle [8, 7, 9, 22, 23, 24, 14, 26, 25, 36] has lead to a set of general-purpose techniques, both for algorithms and for analysis in high dimension. All known algorithms are based on sampling by discrete-time Markov chains. These include the ball walk [20], hit-and-run [33, 26] and the Dikin walk [15], the last requiring stronger access than a membership oracle. In each case, the main challenge is analyzing the mixing time of the Markov chain. For a polytope defined by $m$ inequalities in $\mathbb{R}^{n}$, the current best complexity of sampling is roughly the minimum of $n^{3} \cdot m n$ and $m n \cdot m n^{\omega-1}$ where the first factor in each term is the mixing time and the second factor is the time to implement one step. In fact, the bound of $n^{3}$ on the mixing time (achieved by the ball walk and hit-and-run) holds for arbitrary convex bodies, and $O(m n)$ is just the time to implement a membership oracle. The second term is for the Dikin walk, for which Kannan and Narayanan showed a mixing time of $O(m n)$ for the Dikin walk [15], with each step implementable in roughly matrix multiplication time. For general convex bodies given by membership oracles, $\Omega\left(n^{2}\right)$ is a lower bound on the number of oracle calls for all known walks. A quadratic upper bound would essentially follow from a positive resolution of the KLS hyperplane conjecture (we mention that [3] show a mixing bound of $\tilde{O}\left(n^{2}\right)$ for the ball walk for sampling from a Gaussian distribution restricted to a convex body). The quadratic barrier seems inherent for sampling convex bodies given by membership oracles, holding even for cubes and cylinders for the known walks based on membership oracles. It has not been surpassed thus far even for explicitly described polytopes.

For a polytope in $\mathbb{R}^{n}$, the Euclidean perspective is natural and predominant. The approach so far has been to define a process on the points of the polytope so that the distribution of the current point quickly approaches the uniform (or a desired stationary distribution). The difficulty is that for points near the boundary of a body, steps are necessarily small due to the nature of volume distribution in high dimension. The Dikin walk departs from the standard perspective by making the distribution of the next step depend on the distances of the current point to defining hyperplanes of the polytope. At each step, the process picks a random point from a suitable ellipsoid that is guaranteed to almost lie inside. This process adapts to the boundary, but runs into similar difficulties - the ellipsoid has to shrink as the point approaches the boundary in order to ensure that (a) the stationary distribution is close to uniform and (b) the 1-step distribution is smooth, both necessary properties for proving rapid convergence to the uniform distribution. Even though this walk has the appealing property of being affine-invariant, and thus avoids explicitly rounding the polytope, the current best upper bound for mixing is still quadratic, even for cylinders.

An alternative approach for sampling is the simulation of Brownian motion with boundary reflection $[10,5,4,2]$. While there has been much study of this process, several difficulties arise in turning it into an efficient algorithm. In particular, if the current point is close to the boundary of the polytope, extra care is needed in simulation and the process effectively slows down. However, if it is deep inside the polytope, we should expect that Brownian motion locally looks like a Gaussian distribution and hence it is easier to simulate. This suggests that the standard Euclidean metric, which does not take into account distance to the boundary, is perhaps not the right notion for getting a fast sampling algorithm.

In this paper, we combine the use of Stochastic Differential Equations (SDE) with non-Euclidean geometries (Riemannian manifolds) to break the quadratic barrier for mixing in polytopes. As a result we obtain significantly faster sampling algorithms.

Roughly speaking, our work is based on three key conceptual ideas. The first is the use of a 
Riemannian metric rather than the Euclidean metric. This allows us to scale space as we get closer to the boundary and incorporate boundary information much more smoothly. This idea was already used by Narayanan [28] to extend the Dikin walk to more general classes of convex bodies. The relevant metrics are induced by Hessians of convex barrier functions, objects that have been put to remarkable use for efficient linear and convex optimization [30]. The second idea is to simulate an SDE on the manifold corresponding to the metric, via its geodesics (shortest-path curves, to be defined presently). Unlike straight lines, geodesics bend away from the boundary and this allows us to take larger steps while staying inside the polytope. The third idea is to use a modification of standard Brownian motion via a drift term, i.e., rather than centering the next step at the current point, we first shift the current point deterministically, then take a random step. This drift term compensates the changes of the step size and this makes the process closer to symmetric. Taken together, these ideas allow us to simulate an SDE by a discrete series of ordinary differential equations (ODE), which we show how to solve efficiently to the required accuracy. In order to state our contributions and results more precisely, we introduce some background, under three headings.

Riemannian Geometry. A manifold can be viewed as a surface embedded in a Euclidean space. Each point in the manifold (on the surface), has a tangent space (the linear approximate of the surface at that point) and a local metric. For a point $x$ in a manifold $M$, the metric at $x$ is defined by a positive definite matrix $g(x)$ and the length of a vector $u$ in the tangent space $T_{x} M$ is defined as $\|u\|_{x} \stackrel{\text { def }}{=} u^{T} g(x) u$. By integration, the length of any curve on the manifold is defined as $\int\left\|\frac{d c}{d t}\right\|_{c(t)}$. A basic fact about Riemannian manifolds is that for any point in the manifold, in any direction (from the tangent space), there is locally a shortest path (geodesic) starting in that direction. In Euclidean space, this is just a straight line in the starting direction. Previous random walks involve generating a random direction and going along a straight line in that direction. However such straight lines do not take into account the local geometry, while geodesics do. We give formal definitions in Section 2.1.

Hessian Manifolds. In this paper, we are concerned primarily with Riemannian manifolds induced by Hessians of smooth (infinitely differentiable) strictly convex functions. More precisely, for any such function $\phi$, the local metric (of the manifold induced by $\phi$ ) at a point $x \in M$ is given by the Hessian of $\phi$ at $x$, i.e., $\nabla^{2} \phi(x)$. Since $\phi$ is strictly convex, its Hessian is positive definite and hence the Riemannian manifold induced by $\phi$ is well-defined and is called a Hessian manifold. In the context of convex optimization, we are interested in a class of convex functions called self-concordant barriers. Such convex functions are smooth in a precise sense and blow up on the boundary of a certain convex set. The class of Hessian manifolds corresponding to self-concordant barriers has been studied and used to study interior-point methods (IPM) [16, 31, 29].

Two barriers of particular interest are the logarithmic barrier and the Lee-Sidford (LS) barrier [17], both defined for polytopes. For a polytope $A x>b$, with $A \in \mathbb{R}^{m \times n}$ and $b \in \mathbb{R}^{m}$, for any $x$ in the polytope, the logarithmic barrier is given by

$$
\phi(x)=-\sum_{i} \ln (A x-b)_{i} .
$$

This barrier is efficient in practice and has a self-concordance parameter $\nu \leq m$. The latter controls the number of iterations of the IPM for optimization as $O(\sqrt{\nu})$. The best possible value of $\nu$ is $n$, the dimension. This is achieved up to a constant by the universal barrier [30], the canonical barrier [11] and the entropic barrier [1]. However, these barrier functions take longer to evaluate 
(currently $\Omega\left(n^{5}\right)$ or more). The LS barrier has been shown to be efficiently implementable (in time $\left.O\left(n n z(A)+n^{2}\right)[18]\right)$, while needing only $\tilde{O}(\sqrt{n})$ iterations [17].

In this paper, we develop tools to analyze general Hessian manifolds and show how to use them for the logarithmic barrier to obtain a faster algorithm for sampling polytopes.

Stochastic Differential Equations. Given a self-concordant barrier $\phi$ on a convex set $K$, there is a unique Brownian motion with drift on the Hessian manifold $M$ induced by $\phi$ that has uniform stationary distribution. In the Euclidean coordinate system, the SDE is given by

$$
d x_{t}=\mu\left(x_{t}\right) d t+\left(\nabla^{2} \phi\left(x_{t}\right)\right)^{-1 / 2} d W_{t}
$$

where the first term, called drift, is given by:

$$
\mu_{i}\left(x_{t}\right)=\frac{1}{2} \sum_{j=1}^{n} \frac{\partial}{\partial x_{j}}\left(\left(\nabla^{2} \phi\left(x_{t}\right)\right)^{-1}\right)_{i j} .
$$

This suggests an approach for generating a random point in a polytope, namely to simulate the SDE. The running time of such an algorithm depends on the convergence rate of the SDE and the cost of simulating the SDE in discrete time steps.

Since the SDE is defined on the Riemannian manifold $M$, it is natural to consider the following geodesic walk:

$$
x^{(j+1)}=\exp _{X^{(j)}}\left(\sqrt{h} w+\frac{h}{2} \mu\left(x^{(j)}\right)\right)
$$

where $\exp _{x^{(j)}}$ is a map from $T_{x^{(j)}} M$ back to the manifold, $w$ is a random Gaussian vector on $T_{x^{(j)}} M$, $\mu\left(x^{(j)}\right) \in T_{x^{(j)}} M$ is the drift term and $h$ is the step size. The coefficient of the drift term depends on the coordinate system we use; as we show in Lemma 16, for the coordinate system induced by a geodesic, the drift term is $\mu / 2$ instead of $\mu$ as in (1.1). The Gaussian vector $w$ has mean 0 and variance 1 in the metric at $x$, i.e. for any $u, \mathbb{E}_{w}\langle w, u\rangle_{x}^{2}=\|u\|^{2}$. We write it as $w \sim N_{x}(0, I)$.

It can be shown that this discrete walk converges to (1.1) as $h \rightarrow 0$ and it converges in a rate faster than the walk suggested by Euclidean coordinates, namely, $x^{(j+1)}=x^{(j)}+\sqrt{h} w+h \mu\left(x^{(j)}\right)$. (Note the drift here is proportional to $h$ and not $h / 2$.) This is the reason we study the geodesic walk.

\subsection{Algorithm}

The algorithm is a discrete-time simulation of the geodesic process (1.3). For step-size $h$ chosen in advance, let $p(x \stackrel{w}{\rightarrow} y)$ be the probability density (in Euclidean coordinates) of going from $x$ to $y$ using the local step $w$. In general, the stationary distribution of the geodesic process is not uniform and it is difficult to analyze the stationary distribution unless $h$ is very small, which would lead to a high number of steps. To get around this issue, we use the standard method of rejection sampling to get a uniform stationary distribution. We call this the geodesic walk (see Algo. 2 for full details).

We show how to implement this in Section 3.10. Each iteration of the geodesic walk only uses matrix multiplication and matrix inverse for $O\left(\log ^{(1)} m\right)$ many $O(m) \times O(m)$-size matrices, and the rejection probability is small, i.e., acceptance probability is at least a constant in each step.

To implement the geodesic walk, we need to compute the exponential map $\exp _{x}$, the vector $w^{\prime}$

and the probability densities $p(x \stackrel{w}{\rightarrow} y), p\left(y \stackrel{w^{\prime}}{\rightarrow} x\right)$ efficiently. These computational problems turn out to be similar - all involve solving ordinary differential equations (ODEs) to accuracy $1 / n^{\Theta(1)}$. Hence, one can view the geodesic walk as reducing the problem of simulating an SDE (1.1) to solving 


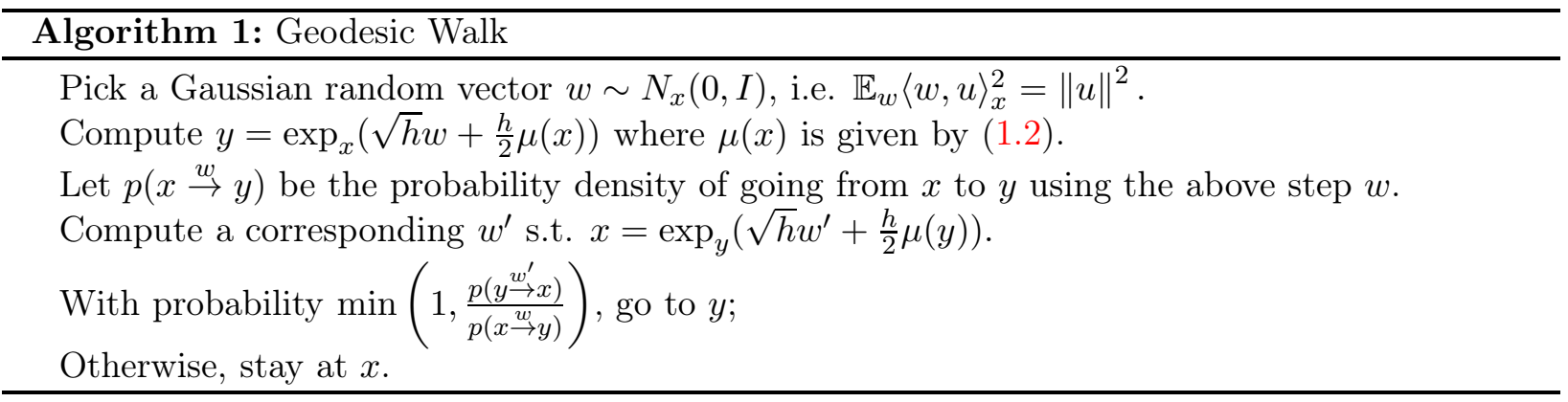

a sequence of ODEs. Although solving ODEs is well-studied, existing literature seems quite implicit about the dependence on the dimension and hence it is difficult to apply it directly. In Section 5, we rederive some existing results about solving ODEs efficiently, but with quantitative estimates of the dependence on the dimension and desired error.

\subsection{Main result}

In this paper, we analyze the geodesic walk for the logarithmic barrier. The convergence analysis will need tools from Riemannian geometry and stochastic calculus, while the implementation uses efficient (approximate) solution of ODEs. Both aspects appear to be of independent interest. For the reader unfamiliar with these topics, we include an exposition of the relevant background.

We analyze the geodesic walk in general and give a bound on the mixing time in terms of a set of manifold parameters (Theorem 23). Applying this to the logarithmic barrier, we obtain a faster sampling algorithm for polytopes, going below the $m n$ mixing time of the Dikin walk, while maintaining the same per-step complexity.

Theorem 1 (Sampling with logarithmic barrier). For any polytope $\{A x \geq b\}$ with $m$ inequalities in $\mathbb{R}^{n}$, the geodesic walk with the logarithmic mixes in $\tilde{O}\left(m n^{\frac{3}{4}}\right)$ steps from a warm start, with each step taking $\tilde{O}\left(m n^{\omega-1}\right)$ time to implement.

The implementation of each step of sampling is based on an efficient algorithm for solving highdimensional ODEs (Theorem 65). We state the implementation as a general theorem below, and expect it will have other applications. As an illustration, we show how Physarum dynamics (studied in [34]) can be computed more efficiently (Section 5.3).

Theorem 2. Let $u(t) \in \mathbb{R}^{n}$ be the solution of the $O D E \frac{d}{d t} u(t)=F(u(t), t), u(0)=v$. Suppose we are given $\varepsilon>0$ and $1 \leq p \leq \infty$ such that

1. There is a degree $d$ polynomial $q$ from $\mathbb{R}$ to $\mathbb{R}^{n}$ such that $q(0)=v$ and $\left\|\frac{d}{d t} u(t)-\frac{d}{d t} q(t)\right\|_{p} \leq \varepsilon$ for all $0 \leq t \leq 1$.

2. For some $L \geq 1$, we have that $\|F(x, t)-F(y, t)\|_{p} \leq L\|x-y\|_{p}$ for all $x, y$ and $0 \leq t \leq 1$.

Then, we can compute $u$ such that $\|u-u(1)\|_{p}=O(\varepsilon)$ in $O\left(n d L^{3} \log ^{2}(d K / \varepsilon)\right)$ time and $O\left(d L^{2} \log (K / \varepsilon)\right)$ evaluations of $F$ where $K=\max _{x, 0 \leq t \leq 1}\|F(x, t)\|_{p}$.

For the application to the geodesic walk, $L=O(1)$ and we did not optimize over the dependence on $L$. 


\subsection{Discussion and open problems}

At a high level, our algorithm is based on the following sequence of fairly natural choices. First, we consider a Brownian motion that gives uniform stationary distribution and such Brownian motion is unique for a given metric (via the Fokker-Planck equation, 15). Since the set we sample is a polytope, we use the metric given by the Hessian of a self-concordant barrier, a well-studied class of metrics in convex optimization. This allows us to reduce the sampling problem to the problem of simulating an SDE. ${ }^{1}$ To simulate an SDE, we apply the Milstein method, well-known in that field. To implement the Milstein method, we perform a change of coordinates to make the metric locally constant, which greatly simplifies the Milstein approximation. These coordinates are called normal coordinates and can be calculated by geodesics (Lemma 16). This gives the step of our walk (1.3).

There are two choices which are perhaps not the most natural. First, it is unclear whether Hessians of self-concordant barriers are the best metrics for the sampling problem; after all, these barriers were designed for solving linear programs. Second, the Milstein method may not be the best choice for discrete approximation. There are other numerical methods with better convergence rates for solving SDEs, such as higher-order Runge-Kutta schemes. However, the latter methods are very complex and it is not clear how to implement them in $\tilde{O}\left(m n^{\omega-1}\right)$ time.

There are several avenues for improving the sampling complexity further. One is to take longer steps and use a higher-order simulation of the SDE that is accurate up to a larger distance. Another part that is not tight in the analysis is the isoperimetry. Our analysis incurs a linear factor in the mixing time due to the isoperimetry. As far as we know, this factor might be as small as $O(1)$. We make this precise via the following, admittedly rash generalization of the KLS hyperplane conjecture. If true, it would directly improve our mixing time bound to $\tilde{O}\left(n^{\frac{3}{4}}\right)$.

Conjecture. For any Hessian manifold $M$ with metric $d$ induced by a convex body $K$, let the isoperimetric ratio of a subset $S$ w.r.t. $d$ be defined as

$$
\psi_{d}(S)=\inf _{\varepsilon>0} \frac{\operatorname{vol}(\{x \in K \backslash S, d(x, S) \leq \varepsilon\})}{\varepsilon \cdot \min \{\operatorname{vol}(S), \operatorname{vol}(K \backslash S)\}}
$$

and the isoperimetric ratio of $d$ as $\psi=\inf _{S \subset K} \psi_{d}(S)$. Then there is a subset $S$ defined as a halfspace intersected with $K$ with $\psi_{d}(S)=O(\psi)$.

We note that we are not simultaneously experts on Riemannian geometry, numerical SDE/ODE and convex geometry; we view our paper as a sampling algorithm that connects different areas while improving the state-of-the-art. Although the sampling problem is a harder problem than solving linear programs, the step size of geodesic walk we use is larger than that of the short-step interior point method. Unlike many papers on manifolds, we do not assume positive curvature everywhere. For example, in recent independent work, Mangoubi and Smith [27] assumed positive sectional curvature bounded between $0<\mathfrak{m}$ and $\mathfrak{M}<\infty$, and an oracle to compute geodesics, and analyzed a geodesic walk for sampling from the uniform distribution on a Riemannian manifold; while their mixing time bound $O\left((\mathfrak{M} / m)^{3}\right)$ is dimension-independent, as far as we know, any manifold that approximates (the double cover of) a polytope well enough would have $\mathfrak{M} / m=\Omega(n)$ and hence does not yield an improvement.

We hope that the connections revealed in this paper might be useful for further development of samplers, linear programming solvers and the algorithmic theory of manifolds.

\footnotetext{
${ }^{1}$ Coincidentally, when we use the best known self-concordant barrier, the canonical barrier, our SDE becomes a Brownian motion with drift on an Einstein manifold. This is similar to how physical particles move under general relativity (an algorithm that has been executed for over 10 billion years!).
} 


\subsection{Outline}

In the next section, we recall the basic definitions and properties of Riemannian geometry and Hessian manifolds, and relevant facts from stochastic calculus and complex analysis. We also derive the discrete-time geodesic walk (in Section 2.2.1), showing how the formula naturally arises. Key concepts that we use repeatedly include geodesics, curvature and Jacobi fields. In Section 3, we prove the convergence guarantee for general Hessian manifolds. The analysis needs three highlevel ingredients: (1) the rejection probability of the filter is small (2) two points that are close in manifold distance have the property that their next step distributions have large overlap (bounded total variation distance) (3) a geometric isoperimetric inequality that shows that large subsets have large boundaries. Of these the last is relatively straightforward, relying on a comparison with the Hilbert metric and existing isoperimetic inequalities for the latter. For the first two, we first derive an explicit formula for the one-step probability distribution (Lemma 27). For bounding the rejection probability, we need to show that this probability is comparable going forwards and going backwards as computed in the algorithm (Theorem 28). The one-step overlap is also derived by comparing the transition probabilities from two nearby points to the same point (Theorem 29). This comparison and resulting bounds depend on several smoothness and stability parameters of the manifold. This part also needs an auxiliary function that controls the change of geodesics locally. An important aspect of the analysis is understanding how this probability changes via the Jacobi fields induced by geodesics. Given these ingredients, the proof of mixing and conductance follows a fairly standard path (Section 3.4). As a warm-up, in Section 3.5, we work out the mixing for a hypercube with the logarithmic barrier — the mixing time is $\tilde{O}\left(n^{\frac{1}{3}}\right)$.

In subsequent sections, we apply this general theorem to the logarithmic barrier for a polytope, to prove Theorem 1. We bound each of the parameters and use an explicit auxiliary function that is just a combination of the infinity norm and the $\ell_{4}$ norm.

The algorithm for solving ODEs (collocation method) is presented and analyzed in Section 5, and this is used to compute the geodesic and transition probabilities. The main idea of the analysis is to show that the ODE can be approximated by a low-degee polynomial, depending on bounds on the derivatives of the solution. To bound these derivatives, we give some general relations for bounding higher derivatives (Section 5.4). As a simple application of the collocation method, we give a faster convergence bound for discretized Physarum dynamics. To apply this method for the $\log$ barrier, in Section 6, we show that the functions we wish to compute (geodesic, transition probability) are complex analytic, then apply the derivative estimates of the previous section and finally bound the time complexity of the collocoation method. 


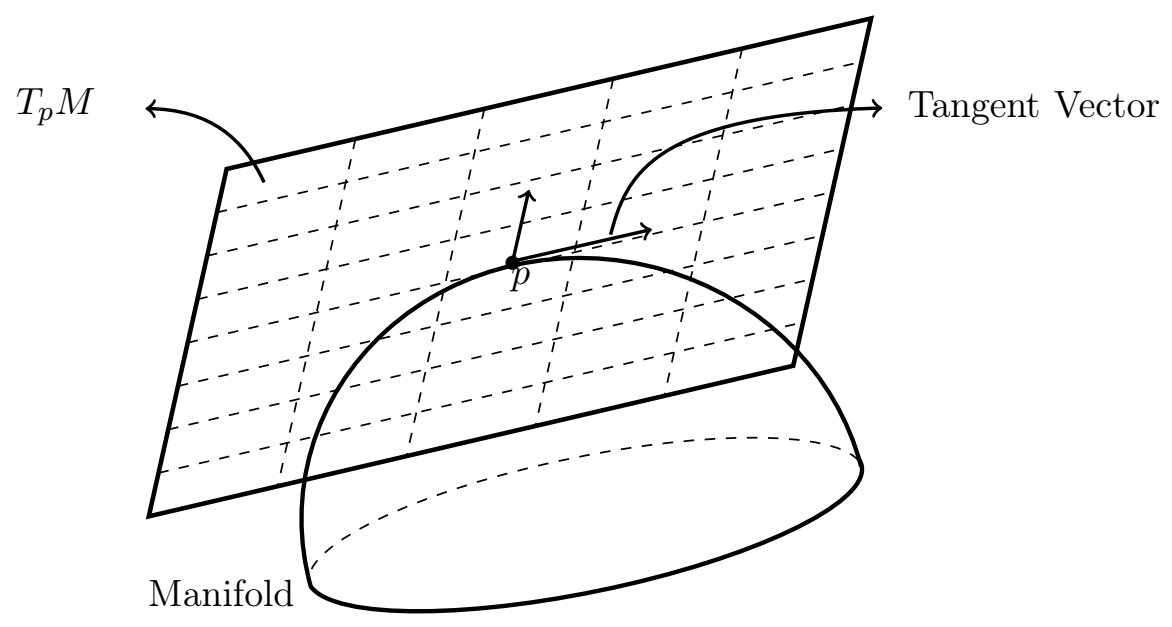

Figure 2.1: Riemannian Manifold and Tangent Space

\section{Background and notation}

Throughout the paper, we use lowercase letter for vectors and vector fields and uppercase letter for matrices and tensors (this is not the convention used in Riemannian geometry). We use $e_{k}$ to denote coordinate vectors. We use $\frac{d}{d t}$ for the usual derivative, e.g. $\frac{d f(c(t))}{d t}$ is the derivative of some function $f$ along a curve $c$ parametrized by $t$, we use $\frac{\partial}{\partial v}$ for the usual partial derivative. We use $D^{k} f(x)\left[v_{1}, v_{2}, \cdots, v_{k}\right]$ for the $k^{t h}$ directional derivative of $f$ at $x$ along $v_{1}, v_{2}, \cdots, v_{k}$. We use $\nabla$ for the connection (manifold derivative, defined below which takes into account the local metric), $D_{v}$ for the directional derivative of vector wrt to the vector (or vector field) $v$ (again, defined below which takes into account the local metric), and $D_{t}$ if the parametrization is clear from the context. We use $g$ for the local metric. Given a point $x \in M, g$ is a matrix with entries $g_{i j}$. Its inverse has entries $g^{i j}$. Also $n$ is the dimension, $m$ the number of inequalities, $\gamma$ is a geodesic, and $\phi$ is a smooth convex function.

\subsection{Basic Definitions of Riemannian geometry}

Here we first introduce basic notions of Riemannian geometry. One can think of a manifold $M$ as a $n$-dimensional "surface" in $\mathbb{R}^{k}$ for some $k \geq n$. In this paper, we only use a special kind of manifolds, called Hessian manifold. For these manifolds, many definition can be defined directly by some mysterious formulas. If it helps, the reader can use this section merely to build intuition and use Lemma 10 instead as the formal definition of various concepts defined here.

1. Tangent space $T_{p} M$ : For any point $p$, the tangent space $T_{p} M$ of $M$ at point $p$ is a linear subspace of $\mathbb{R}^{k}$ of dimension $n$. Intuitively, $T_{p} M$ is the vector space of possible directions that are tangential to the manifold at $x$. Equivalently, it can be thought as the first-order linear approximation of the manifold $M$ at $p$. For any curve $c$ on $M$, the direction $\frac{d}{d t} c(t)$ is tangent to $M$ and hence lies in $T_{c(t)} M$. When it is clear from context, we define $c^{\prime}(t)=\frac{d c}{d t}(t)$. For any open subset $M$ of $\mathbb{R}^{n}$, we can identify $T_{p} M$ with $\mathbb{R}^{n}$ because all directions can be realized by derivatives of some curves in $\mathbb{R}^{n}$.

2. Riemannian metric: For any $v, u \in T_{p} M$, the inner product (Riemannian metric) at $p$ is given by $\langle v, u\rangle_{p}$ and this allows us to define the norm of a vector $\|v\|_{p}=\sqrt{\langle v, v\rangle_{p}}$. We call a 
manifold a Riemannian manifold if it is equipped with a Riemannian metric. When it is clear from context, we define $\langle v, u\rangle=\langle v, u\rangle_{p}$. In $\mathbb{R}^{n},\langle v, u\rangle_{p}$ is the usual $\ell_{2}$ inner product.

3. Differential (Pushforward) $d$ : Given a function $f$ from a manifold $M$ to a manifold $N$, we define $d f(x)$ as the linear map from $T_{x} M$ to $T_{f(x)} N$ such that

$$
d f(x)\left(c^{\prime}(0)\right)=(f \circ c)^{\prime}(0)
$$

for any curve $c$ on $M$ starting at $x=c(0)$. When $M$ and $N$ are Euclidean spaces, $d f(x)$ is the Jacobian of $f$ at $x$. We can think of pushforward as a manifold Jacobian, i.e., the first-order approximation of a map from a manifold to a manifold.

4. Hessian manifold: We call $M$ a Hessian manifold (induced by $\phi$ ) if $M$ is an open subset of $\mathbb{R}^{n}$ with the Riemannian metric at any point $p \in M$ defined by

$$
\langle v, u\rangle_{p}=v^{T} \nabla^{2} \phi(p) u
$$

where $v, u \in T_{p} M$ and $\phi$ is a smooth convex function on $M$.

5. Length: For any curve $c:[0,1] \rightarrow M$, we define its length by

$$
L(c)=\int_{0}^{1}\left\|\frac{d}{d t} c(t)\right\|_{c(t)} d t
$$

6. Distance: For any $x, y \in M$, we define $d(x, y)$ be the infimum of the lengths of all paths connecting $x$ and $y$. In $\mathbb{R}^{n}, d(x, y)=\|x-y\|_{2}$.

7. Geodesic: We call a curve $\gamma(t):[a, b] \rightarrow M$ a geodesic if it satisfies both of the following conditions:

(a) The curve $\gamma(t)$ is parameterized with constant speed. Namely, $\left\|\frac{d}{d t} \gamma(t)\right\|_{\gamma(t)}$ is constant for $t \in[a, b]$.

(b) The curve is the locally shortest length curve between $\gamma(a)$ and $\gamma(b)$. Namely, for any family of curve $c(t, s)$ with $c(t, 0)=\gamma(t)$ and $c(a, s)=\gamma(a)$ and $c(b, s)=\gamma(b)$, we have that $\left.\frac{d}{d s}\right|_{s=0} \int_{a}^{b}\left\|\frac{d}{d t} c(t, s)\right\|_{c(t, s)} d t=0$.

Note that, if $\gamma(t)$ is a geodesic, then $\gamma(\alpha t)$ is a geodesic for any $\alpha$. Intuitively, geodesics are local shortest paths. In $\mathbb{R}^{n}$, geodesics are straight lines.

8. Exponential map: The map $\exp _{p}: T_{p} M \rightarrow M$ is defined as

$$
\exp _{p}(v)=\gamma_{v}(1)
$$

where $\gamma_{v}$ is the unique geodesic starting at $p$ with initial velocity $\gamma_{v}^{\prime}(0)$ equal to $v$. The exponential map takes a straight line $t v \in T_{p} M$ to a geodesic $\gamma_{t v}(1)=\gamma_{v}(t) \in M$. Note that $\exp _{p}$ maps $v$ and $t v$ to points on the same geodesic. Intuitively, the exponential map can be thought as point-vector addition in a manifold. In $\mathbb{R}^{n}$, we have $\exp _{p}(v)=p+v$. 
9. Parallel transport: Given any geodesic $c(t)$ and a vector $v$ such that $\left\langle v, c^{\prime}(0)\right\rangle_{c(0)}=0$, we define the parallel transport of $v$ along $c(t)$ by the following process: Take $h$ to be infinitesimally small and $v_{0}=v$. For $i=1,2, \cdots, 1 / h$, we let $v_{i h}$ be the vector orthogonal to $c^{\prime}(i h)$ that minimizes the distance on the manifold between $\exp _{c(i h)}\left(h v_{i h}\right)$ and $\exp _{c((i-1) h)}\left(h v_{(i-1) h}\right)$. Intuitively, the parallel transport finds the vectors on the curve such that their end points are closest to the end points of $v$. For general vector $v \in T_{c^{\prime}(0)}$, we write $v=\alpha c^{\prime}(0)+w$ and we define the parallel transport of $v$ along $c(t)$ is the sum of $\alpha c^{\prime}(t)$ and the parallel transport of $w$ along $c(t)$. For non-geodesic curve, see the definition in Fact 3.

10. Orthonormal frame: Given vector fields $v_{1}, v_{2}, \cdots, v_{n}$ on a subset of $M$, we call $\left\{v_{i}\right\}_{i=1}^{n}$ is an orthonormal frame if $\left\langle v_{i}, v_{j}\right\rangle_{x}=\delta_{i j}$ for all $x$. Given a curve $c(t)$ and an orthonormal frame at $c(0)$, we can extend it on the whole curve by parallel transport and it remains orthonormal on the whole curve.

11. Directional derivatives and the Levi-Civita connection: For a vector $v \in T_{p} M$ and a vector field $u$ in a neighborhood of $p$, let $\gamma_{v}$ be the unique geodesic starting at $p$ with initial velocity $\gamma_{v}^{\prime}(0)=v$. Define

$$
\nabla_{v} u=\lim _{h \rightarrow 0} \frac{u(h)-u(0)}{h}
$$

where $u(h) \in T_{p} M$ is the parallel transport of $u(\gamma(h))$ from $\gamma(h)$ to $\gamma(0)$. Intuitively, LeviCivita connection is the directional derivative of $u$ along direction $v$, taking the metric into account. In particular, for $\mathbb{R}^{n}$, we have $\nabla_{v} u(x)=\frac{d}{d t} u(x+t v)$. When $u$ is defined on a curve $c$, we define $D_{t} u=\nabla_{c^{\prime}(t)} u$. In $\mathbb{R}^{n}$, we have $D_{t} u(\gamma(t))=\frac{d}{d t} u(\gamma(t))$. We reserve $\frac{d}{d t}$ for the usual derivative with Euclidean coordinates.

We list some basic facts about the definitions introduced above that are useful for computation and intuition.

Fact 3. Given a manifold $M$, a curve $c(t) \in M$, a vector $v$ and vector fields $u, w$ on $M$, we have the following:

1. (alternative definition of parallel transport) $v(t)$ is the parallel transport of $v$ along $c(t)$ if and only if $\nabla_{c^{\prime}(t)} v(t)=0$.

2. (alternative definition of geodesic) $c$ is a geodesic if and only if $\nabla_{c^{\prime}(t)} c^{\prime}(t)=0$.

3. (linearity) $\nabla_{v}(u+w)=\nabla_{v} u+\nabla_{v} w$.

4. (product rule) For any scalar-valued function $f, \nabla_{v}(f \cdot u)=\frac{\partial f}{\partial v} u+f \cdot \nabla_{v} u$.

5. (metric preserving) $\frac{d}{d t}\langle u, w\rangle_{c(t)}=\left\langle D_{t} u, w\right\rangle_{c(t)}+\left\langle u, D_{t} w\right\rangle_{c(t)}$.

6. (torsion free-ness) For any map $c(t, s)$ from a subset of $\mathbb{R}^{2}$ to $M$, we have that $D_{s} \frac{\partial c}{\partial t}=D_{t} \frac{\partial c}{\partial s}$ where $D_{s}=\nabla_{\frac{\partial c}{\partial s}}$ and $D_{t}=\nabla_{\frac{\partial c}{\partial t}}$.

7. (alternative definition of Levi-Civita connection) $\nabla_{v} u$ is the unique linear mapping from the product of vector and vector field to vector field that satisfies (3), (4), (5) and (6). 

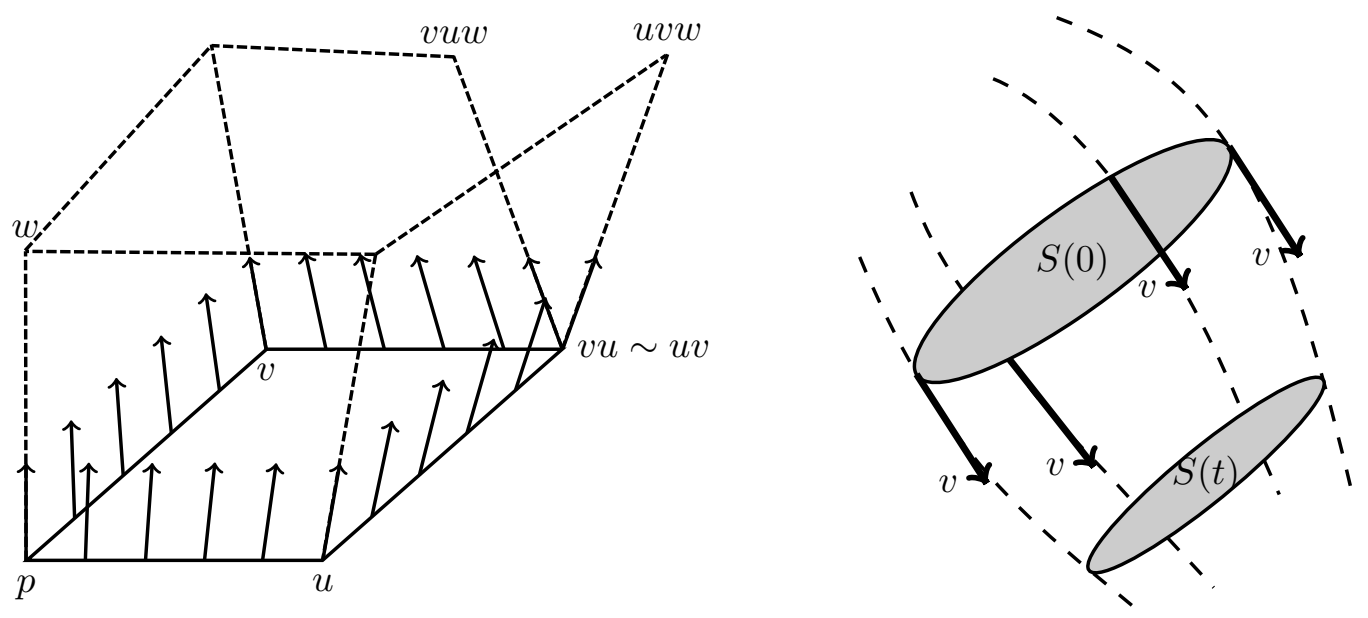

Figure 2.2: Riemann curvature tensor measures the deviation of parallelepipeds (2.1) and Ricci curvature measures the change of volumes (2.2). The illustrations are inspired by [32].

\subsubsection{Curvature}

Here, we define various notions of curvature. Roughly speaking, they measure the amount by which a manifold deviates from Euclidean space.

Given vector $u, v \in T_{p} M$, in this section, we define $u v$ be the point obtained from moving from $p$ along direction $u$ with distance $\|u\|_{p}$ (using geodesic), then moving along direction " $v$ " with distance $\|v\|_{p}$ where " $v$ " is the parallel transport of $v$ along the path $u$. In $\mathbb{R}^{n}$, $u v$ is exactly $p+u+v$ and hence $u v=v u$, namely, parallelograms close up. For a manifold, parallelograms almost close up, namely, $d(u v, v u)=o(\|u\|\|v\|)$. This property is called being torsion-free.

1. Riemann curvature tensor: Three-dimensional parallelepipeds might not close up, and the curvature tensor measures how far they are from closing up. Given vector $u, v, w \in T_{p} M$, we define $u v w$ as the point obtained by moving from $u v$ along direction " $w$ " for distance $\|w\|_{p}$ where " $w$ " is the parallel transport of $w$ along the path $u v$. In a manifold, parallelepipeds do not close up and the Riemann curvature tensor how much uvw deviates from vuw. Formally, for vector fields $v, w$, we define $\tau_{v} w$ be the parallel transport of $w$ along the vector field $v$ for one unit of time. Given vector field $v, w, u$, we define the Riemann curvature tensor by

$$
R(u, v) w=\left.\frac{d}{d s} \frac{d}{d t} \tau_{s u}^{-1} \tau_{t v}^{-1} \tau_{s u} \tau_{t v} w\right|_{t, s=0}
$$

Riemann curvature tensor is a tensor, namely, $R(u, v) w$ at point $p$ depends only on $u(p), v(p)$ and $w(p)$.

2. Ricci curvature: Given a vector $v \in T_{p} M$, the Ricci curvature $\operatorname{Ric}(v)$ measures if the geodesics starting around $p$ with direction $v$ converge together. Positive Ricci curvature indicates the geodesics converge while negative curvature indicates they diverge. Let $S(0)$ be a small shape around $p$ and $S(t)$ be the set of point obtained by moving $S(0)$ along geodesics in the direction $v$ for $t$ units of time. Then,

$$
\operatorname{vol} S(t)=\operatorname{vol} S(0)\left(1-\frac{t^{2}}{2} \operatorname{Ric}(v)+\text { smaller terms }\right) .
$$


Formally, we define

$$
\operatorname{Ric}(v)=\sum_{u_{i}}\left\langle R\left(v, u_{i}\right) u_{i}, v\right\rangle
$$

where $u_{i}$ is an orthonormal basis of $T_{p} M$. Equivalently, we have $\operatorname{Ric}(v)=\mathbb{E}_{u \sim N(0, I)}\langle R(v, u) u, v\rangle$. For $\mathbb{R}^{n}, \operatorname{Ric}(v)=0$. For a sphere in $n+1$ dimension with radius $r, \operatorname{Ric}(v)=\frac{n-1}{r^{2}}\|v\|^{2}$.

Fact 4 (Alternative definition of Riemann curvature tensor). Given any $M$-valued function $c(t, s)$, we have vector fields $\frac{\partial c}{\partial t}$ and $\frac{\partial c}{\partial s}$ on $M$. Then, for any vector field $z$,

$$
R\left(\frac{\partial c}{\partial t}, \frac{\partial c}{\partial s}\right) z=\nabla_{\frac{\partial c}{\partial t}} \nabla_{\frac{\partial c}{\partial s}} z-\nabla_{\frac{\partial c}{\partial s}} \nabla_{\frac{\partial c}{\partial t}} z
$$

Equivalently, we write $R\left(\partial_{t} c, \partial_{s} c\right) z=D_{t} D_{s} z-D_{s} D_{t} z$.

Fact 5. Given vector fields $v, u, w, z$ on $M$,

$$
\langle R(v, u) w, z\rangle=\langle R(w, z) v, u\rangle=-\langle R(u, v) w, z\rangle=-\langle R(v, u) z, w\rangle .
$$

\subsubsection{Jacobi field}

In this paper, we often study the behavior of a family of geodesics. One crucial fact we use is that the change of a family of geodesics satisfies the following equation, called the Jacobi equation:

Theorem 6 ([13, Thm 4.2.1]). Let $c:[0, \ell] \rightarrow M$ be a geodesic and $c(t, s)$ be a variation of $c(t)$ (i.e. $c(t, 0)=c(t))$ such that $c_{s}(t) \stackrel{\text { def }}{=} c(t, s)$ is a geodesic for all $s$. Then $\left.u(t) \stackrel{\text { def }}{=} \frac{\partial}{\partial s} c(t, s)\right|_{s=0}$ satisfies the following equation

$$
D_{t} D_{t} u+R\left(u, \frac{d c}{d t}\right) \frac{d c}{d t}=0
$$

where $R(\cdot, \cdot) \cdot$ is Riemann curvature tensor defined before. Conversely, any vector field $V$ on $c(t)$ satisfying the equation

$$
D_{t} D_{t} V+R\left(V, \frac{d c}{d t}\right) \frac{d c}{d t}=0
$$

can be obtained by a variation of $c(t)$ through geodesics. We call any vector field satisfying this equation a Jacobi field.

See figure 2.3 for an illustration.

In the appendix, we prove the first part of the theorem above as an illustration of the Jacobi equation. In $\mathbb{R}^{n}$, geodesics are straight lines and a Jacobi field is linear, namely, $u(t)=u(0)+u^{\prime}(0) t$. A similar decomposition holds for any Jacobi field.

Fact 7. Given a unit speed geodesic $c(t)$, every Jacobi field $u(t)$ on $c(t)$ can be split into a tangential part $u_{1}$ and a normal part $u_{2}$ such that

1. $u=u_{1}+u_{2}$,

2. $u_{1}$ and $u_{2}$ are Jacobi fields, namely, $D_{t} D_{t} u_{1}+R\left(u_{1}, \frac{d c}{d t}\right) \frac{d c}{d t}=0$ and $D_{t} D_{t} u_{2}+R\left(u_{2}, \frac{d c}{d t}\right) \frac{d c}{d t}=0$,

3. $u_{1}$ is parallel to $\frac{d c}{d t}$ and is linear, namely, $u_{1}(t)=\left(\left\langle u(0), \frac{d c}{d t}(0)\right\rangle_{c(0)}+\left\langle D_{t} u(0), \frac{d c}{d t}(0)\right\rangle_{c(0)} t\right) \frac{d c}{d t}(t)$,

4. $u_{2}$ is orthogonal to $\frac{d c}{d t}$, namely, $\left\langle u_{2}(t), \frac{d c}{d t}(t)\right\rangle_{c(t)}=0$. 


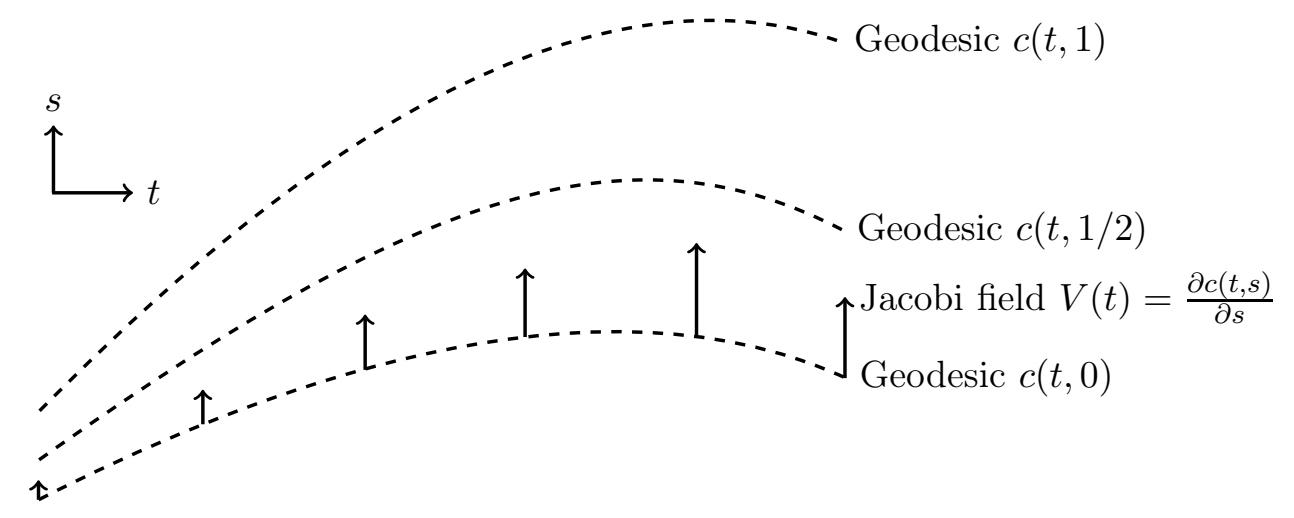

Figure 2.3: A Jacobi field describes the difference between geodesic.

Definition 8. Given a geodesic $\gamma(t)$, we define a linear map $R(t): T_{\gamma(t)} M \rightarrow T_{\gamma(t)} M$ by

$$
R(t) u=R\left(u, \gamma^{\prime}(t)\right) \gamma^{\prime}(t) .
$$

In particular, the Jacobi equation on $\gamma(t)$ can be written as $D_{t}^{2} u+R(t) u=0$.

Given a coordinate system $x_{i}$, the linear map $R(t)$ can be written as a symmetric matrix

$$
R(t)_{i j}=\left\langle R\left(x_{i}(t), \gamma^{\prime}(t)\right) \gamma^{\prime}(t), x_{j}(t)\right\rangle .
$$

To analysis the Jacobi equation, it is convenient to adopt the follow matrix notation.

Definition 9. Given a linear map $A: T_{x} M \rightarrow T_{x} M$, we define $\|A\|_{2}=\max _{\|v\|_{x}=1}\|A v\|_{x},\|A\|_{F}=$ $\sum_{i, j}\left\langle v_{i}, A v_{j}\right\rangle_{x}^{2}$ and $\operatorname{Tr} A=\sum_{i} v_{i} A v_{i}$ where $\left\{v_{i}\right\}_{i=1}^{n}$ is some arbitrary orthonormal basis of $T_{x} M$.

In particular, we have that $\operatorname{Ric}\left(\gamma^{\prime}(t)\right)=\operatorname{Tr} R(t)$.

\subsubsection{Hessian manifolds}

Recall that a manifold is called Hessian if it is a subset of $\mathbb{R}^{n}$ and its metric is given by $g_{i j}=\frac{\partial^{2}}{\partial x^{i} \partial x^{j}} \phi$ for some smooth convex function $\phi$. We let $g^{i j}$ be entries of the inverse matrix of $g_{i j}$. For example, we have $\sum_{j} g^{i j} g_{j k}=\delta_{i k}$. We use $\phi_{i j}$ to denote $\frac{\partial^{2}}{\partial x^{i} \partial x^{j}} \phi$ and $\phi_{i j k}$ to denote $\frac{\partial^{3}}{\partial x^{i} \partial x^{j} \partial x^{k}} \phi$.

Since a Hessian manifold is a subset of Euclidean space, we identify tangent spaces $T_{p} M$ by Euclidean coordinates. The following lemma gives formulas for the Levi-Civita connection and curvature under Euclidean coordinates.

Lemma 10 ([35]). Given a Hessian manifold $M$, vector fields $v, u, w, z$ on $M$, we have the following:

1. (Levi-Civita connection) $\nabla_{v} u=\sum_{i k} v_{i} \frac{\partial u_{k}}{\partial x_{i}} e_{k}+\sum_{i j k} v_{i} u_{j} \Gamma_{i j}^{k} e_{k}$ where $e_{k}$ are coordinate vectors and the Christoffel symbol

$$
\Gamma_{i j}^{k}=\frac{1}{2} \sum_{l} g^{k l} \phi_{i j l}
$$

2. (Riemann curvature tensor) $\langle R(u, v) w, z\rangle=\sum_{i j l k} R_{k l i j} u_{i} v_{j} w_{l} z_{k}$ where

$$
R_{k l i j}=\frac{1}{4} \sum_{p q} g^{p q}\left(\phi_{j k p} \phi_{i l q}-\phi_{i k p} \phi_{j l q}\right) .
$$


3. (Ricci curvature) $\operatorname{Ric}(v)=\frac{1}{4} \sum_{i j l k p q} g^{p q} g^{j l}\left(\phi_{j k p} \phi_{i l q}-\phi_{i k p} \phi_{j l q}\right) v_{i} v_{k}$.

As an exercise, the reader can try to prove Fact 3 for Hessian manifolds using the above lemma as a definition. The proof is given in the appendix.

In this paper, geodesics are everywhere and we will be using the following lemma in all of our calculations.

Lemma 11 ([31, Cor 3.1]). If $\phi$ is a self-concordant function, namely, $\left|D^{3} f(x)[h, h, h]\right| \leq 2\left(D^{2} f(x)[h, h]\right)^{3 / 2}$, then the corresponding Hessian manifold $M$ is geodesically complete, namely, for any $p \in M$, the exponential map is defined on the entire tangent space $T_{p} M$ and for any two points $p, q \in M$, there is a length minimizing geodesic connecting them.

In particular, for a polytope $M=\{x: A x>b\}$, the Hessian manifold induced by the function $\phi(x)=-\sum_{i} \log \left(a_{i}^{T} x-b_{i}\right)$ is geodesically complete.

\subsubsection{Normal coordinates}

For any manifold $M$, and any $p \in M$, the exponential map $\exp _{x}$ maps from $T_{x} M$ to $M$. Since $T_{x} M$ is isomorphic to $\mathbb{R}^{n}$, $\exp _{x}^{-1}$ gives a local coordinate system of $M$ around $x$. We call this system the normal coordinates at $x$. In a normal coordinate system, the metric is locally constant.

Lemma 12. In normal coordinates, we have

$$
g_{i j}(x)=\delta_{i j}-\frac{1}{3} \sum_{k l} R_{i k j l}(x) x^{k} x^{l}+O\left(|x|^{3}\right) .
$$

For a Hessian manifold, one can do a linear transformation to make the normal coordinates coincide with Euclidean coordinates up to the first order.

Lemma 13. Given a Hessian manifold $M$ and any point $x \in M$. We pick a basis of $T_{x} M$ such that

$$
\exp _{x}(t v)=x+t v+O\left(t^{2}\right) .
$$

Let $F: M \rightarrow \mathbb{R}^{n}$ be the normal coordinates defined by $F(y)=\exp _{x}^{-1}$. Then, we

$$
D F[h]=h \text { and } D^{2} F_{k}(x)[h, h]=h^{T} \Gamma^{k} h
$$

where $F_{k}$ is the $k^{\text {th }}$ coordinate of $F$ and $\Gamma^{k}$ is the matrix with entries $\Gamma_{i j}^{k}$ defined in Lemma 10.

\section{$2.2 \quad$ Stochastic calculus}

A stochastic differential equation (SDE) describes a stochastic process over a domain $\Omega$. It has the form $d x_{t}=\mu\left(x_{t}, t\right) d t+\sigma\left(x_{t}, t\right) d W_{t}$ where $x_{t}$ is the current point at time $t, W_{t}$ is a standard Brownian motion, and $\mu\left(x_{t}, t\right), \sigma\left(x_{t}, t\right)$ are the mean and covariance of the next infinitesimal step at time $t$.

Lemma 14 (Itō's lemma). Given a SDE $d x_{t}=\mu\left(x_{t}\right) d t+\sigma\left(x_{t}\right) d W_{t}$ and any smooth function $f$, we have

$$
d f\left(t, x_{t}\right)=\left\{\frac{\partial f}{\partial t}+\langle\nabla f, \mu\rangle+\frac{1}{2} \operatorname{Tr}\left[\sigma^{T}\left(\nabla^{2} f\right) \sigma\right]\right\} d t+(\nabla f)^{T} \sigma d W_{t}
$$


SDEs are closely related to diffusion equations:

$$
\frac{\partial}{\partial t} p(x, t)=\frac{1}{2} \nabla \cdot(A(x, t) \nabla p(x, t))
$$

where $p(x, t)$ is the density at point $x$ and time $t, \nabla$. is the usual divergence operator, $\nabla p$ is the gradient of $p$ and the matrix $A(x, t)$ represents the diffusion coefficient at point $x$ and time $t$. When $A(x, t)=I$, we get the familiar heat equation:

$$
\frac{\partial}{\partial t} p(x, t)=\frac{1}{2} \Delta p(x, t)
$$

In this paper, the diffusion coefficient will be a symmetric positive definite matrix given by the Hessian $\left(\nabla^{2} \phi(x)\right)^{-1}$ of a convex function $\phi(x)$.

The Fokker-Planck equation connects an SDE to a diffusion equation.

Theorem 15 (Fokker-Planck equation). For any stochastic differential equation (SDE) of the form

$$
d x_{t}=\mu\left(x_{t}, t\right) d t+\sqrt{A\left(x_{t}, t\right)} d W_{t},
$$

the probability density of the SDE is given by the diffusion equation

$$
\frac{\partial}{\partial t} p(x, t)=-\sum_{i=1}^{n} \frac{\partial}{\partial x_{i}}\left[\mu_{i}(x, t) p(x, t)\right]+\frac{1}{2} \sum_{i=1}^{n} \sum_{j=1}^{n} \frac{\partial^{2}}{\partial x_{i} x_{j}}\left[A_{i j}(x, t) p(x, t)\right] .
$$

\subsubsection{Derivation of the Geodesic walk}

Given a smooth convex function $\phi$ on the convex domain $M$, namely that it is convex and is infinitely differentiable at every interior point of $M$, we consider the corresponding diffusion equation

$$
\frac{\partial}{\partial t} p(x, t)=\frac{1}{2} \nabla \cdot\left(\nabla^{2} \phi\right)^{-1} \nabla p
$$

We can expand it by

$$
\begin{aligned}
\frac{\partial}{\partial t} p(x, t) & =\frac{1}{2} \sum_{i=1}^{n} \frac{\partial}{\partial x_{i}}\left(\sum_{j=1}^{n}\left(\left(\nabla^{2} \phi\right)^{-1}\right)_{i j} \frac{\partial}{\partial x_{j}} p(x, t)\right) \\
& =\frac{1}{2} \sum_{i=1}^{n} \sum_{j=1}^{n} \frac{\partial^{2}}{\partial x_{i} x_{j}}\left(\left(\left(\nabla^{2} \phi\right)^{-1}\right)_{i j} p(x, t)\right)-\frac{1}{2} \sum_{i=1}^{n} \sum_{j=1}^{n} \frac{\partial}{\partial x_{i}}\left(\frac{\partial}{\partial x_{j}}\left(\left(\nabla^{2} \phi\right)^{-1}\right)_{i j} p(x, t)\right) .
\end{aligned}
$$

The uniform distribution is the stationary distribution of this diffusion equation. Now applying the Fokker-Planck equation (Theorem 15) with $A=\left(\nabla^{2} \phi\right)^{-1}$, the SDE for the above diffusion is given by:

$$
d x_{t}=\mu\left(x_{t}\right) d t+\left(\nabla^{2} \phi\left(x_{t}\right)\right)^{-1 / 2} d W_{t} .
$$

This explains the definition of (1.1). To simplify the notation, we write the SDE as

$$
d x_{t}=\mu\left(x_{t}\right) d t+\sigma\left(x_{t}\right) d W_{t}
$$

where $\sigma\left(x_{t}\right)=\left(\nabla^{2} \phi\left(x_{t}\right)\right)^{-1 / 2}$. One way to simulate this is via the Euler-Maruyama method, namely

$$
x_{(t+1) h}=x_{t h}+\mu\left(x_{t h}\right) h+\sigma\left(x_{t h}\right) w_{t h} \sqrt{h}
$$


where $w_{t h} \sim N_{x_{t h}}(0, I)$. We find the direction we are heading and take a small step along that direction. However, if we view $M$ as a manifold, then directly adding the direction $\mu\left(x_{t h}\right) h+$ $\sigma\left(x_{t h}\right) w_{t h} \sqrt{h}$ to $x_{t h}$ is not natural; the Euclidean coordinate is just an arbitrary coordinate system and we could pick any other coordinate systems and add the direction into $x_{t h}$, giving a different step. Instead, we take the step in normal coordinates (Section 2.1.4).

In particular, given an initial point $x_{0}$, we define $F=\exp _{x_{0}}^{-1}$ and we note that $F\left(x_{t}\right)$ is another SDE. To see the defining equation of this transformed SDE, we use Itō's lemma (Lemma 14) to show that the transformed SDE looks the same but with half the drift term. This explains the formulation of geodesic walk: $x^{(j+1)}=\exp _{x^{(j)}}\left(\sqrt{h} w+\frac{h}{2} \mu\left(x^{(j)}\right)\right)$.

Lemma 16. Let $F=\exp _{x_{0}}^{-1}$ and $x_{t}$ satisfies the $S D E$ (2.3) Then we have

$$
d F\left(x_{0}\right)=\frac{1}{2} \mu\left(x_{0}\right) d t+\sigma\left(x_{0}\right) d W_{0} .
$$

Proof. Itō's lemma (Lemma 14) shows that

$$
d F_{k}\left(x_{t}\right)=\left\{\left\langle\nabla F_{k}, \mu\right\rangle+\frac{1}{2} \operatorname{Tr}\left[\sigma^{T}\left(\nabla^{2} F_{k}\right) \sigma\right]\right\} d t+\left(\nabla F_{k}\right)^{T} \sigma d W_{t}
$$

where $F_{k}$ indicates the $k^{\text {th }}$ coordinate of $F$.

From Lemma 13, we have that $\left\langle\nabla F_{k}\left(x_{0}\right), \mu\right\rangle=\mu_{k},\left(\nabla F_{k}\left(x_{0}\right)\right)^{T} \sigma=e_{k}^{T} \sigma$ and

$$
\begin{aligned}
\operatorname{Tr}\left[\sigma\left(x_{0}\right)^{T}\left(\nabla^{2} F_{k}\left(x_{0}\right)\right) \sigma\left(x_{0}\right)\right] & =\sum_{i} D^{2} F_{k}\left[\sigma e_{i}, \sigma e_{i}\right] \\
& =\sum_{i} e_{i}^{T} \sigma^{T} \Gamma^{k} \sigma e_{i} \\
& =\operatorname{Tr}\left(\sigma^{T} \Gamma^{k} \sigma\right) \\
& =\sum e_{i}^{T} \Gamma^{k}\left(\nabla^{2} \phi\right)^{-1} e_{i} .
\end{aligned}
$$

Now, using Lemma 10, we have that

$$
\operatorname{Tr}\left[\sigma\left(x_{0}\right)^{T}\left(\nabla^{2} F_{k}\left(x_{0}\right)\right) \sigma\left(x_{0}\right)\right]=\frac{1}{2} \sum_{i j l} g^{k l} \phi_{i j l} g^{j i}
$$

Hence, we have that

$$
d F_{k}\left(x_{0}\right)=\left\{\mu_{k}+\frac{1}{4} \sum_{i j l} g^{k l} \phi_{i j l} g^{j i}\right\} d t+e_{k}^{T} \sigma d W_{t}
$$

Recall that the drift term (1.2) is given by

$$
\begin{aligned}
\mu_{k} & =\frac{1}{2} \sum_{i=1}^{n} \frac{\partial}{\partial x_{i}}\left(\left(\nabla^{2} \phi\right)^{-1}\right)_{k i} \\
& =-\frac{1}{2} \sum_{i} e_{k}^{T}\left(\nabla^{2} \phi\right)^{-1} \frac{\partial}{\partial x_{i}} \nabla^{2} \phi\left(\nabla^{2} \phi\right)^{-1} e_{i} \\
& =-\frac{1}{2} \sum_{l, i, j} g^{k l} \frac{\partial}{\partial x_{i}} \phi_{l j} g^{j i} \\
& =-\frac{1}{2} \sum_{i j l} g^{k l} \phi_{i j l} g^{j i} .
\end{aligned}
$$

Therefore, we have the result. 
To understand why Euler-Maruyama method works especially better on normal coordinates, we recall the following theorem:

Theorem 17 (Euler-Maruyama method). Given a SDE $d x_{t}=\mu\left(x_{t}\right) d t+\sigma\left(x_{t}\right) d W_{t}$ where both $\mu\left(x_{t}\right) \in \mathbb{R}^{d}$ and $\sigma\left(x_{t}\right) \in \mathbb{R}^{d \times m}$ are Lipschitz smooth functions, consider the algorithm

$$
\begin{aligned}
\bar{x}_{(i+1) h} & =\bar{x}_{i h}+\mu\left(\bar{x}_{i h}\right) h+\sigma\left(\bar{x}_{i h}\right)\left(W_{(i+1) h}-W_{i h}\right) \text { for all } i \geq 0 \\
\bar{x}_{0} & =x_{0} .
\end{aligned}
$$

For some small enough $h>0$, we have that

$$
\mathbb{E}\left\|\bar{x}_{1}-x_{1}\right\|=O(\sqrt{h}) .
$$

As a comparison, it is known that there is better method such as the following that gives better error.

Theorem 18 (Milstein method). Given a SDE $d x_{t}=\mu\left(x_{t}\right) d t+\sigma\left(x_{t}\right) d W_{t}$ where both $\mu\left(x_{t}\right) \in \mathbb{R}^{d}$ and $\sigma\left(x_{t}\right) \in \mathbb{R}^{d \times m}$ are Lipschitz smooth functions, consider the algorithm

$$
\begin{aligned}
\bar{x}_{(i+1) h} & =\bar{x}_{i h}+\mu\left(\bar{x}_{i h}\right) h+\sigma\left(\bar{x}_{i h}\right)\left(W_{(i+1) h}-W_{i h}\right)+\sum_{j_{1}, j_{2}=1}^{m} L_{i}^{j_{1}} \sigma^{k, j_{2}}\left(\bar{x}_{i h}\right) I_{j_{1} j_{2}, i} \text { for all } i \geq 0 \\
\bar{x}_{0} & =x_{0} .
\end{aligned}
$$

where $L_{i}^{j}=\sum_{k=1}^{d} \sigma^{k, j}\left(\bar{x}_{i h}\right) \frac{\partial}{\partial x^{k}}$ and $I_{j_{1} j_{2}, i} \sim \int_{0}^{h} \int_{i h}^{i h+t_{1}} d W_{t_{2}}^{j_{2}} d W_{t_{1}}^{j_{1}}$. For some small enough $h>0$, we have that

$$
\mathbb{E}\left\|\bar{x}_{1}-x_{1}\right\|=O(h) .
$$

Note that under normal coordinates, the metric $\sigma$ is locally constant (Lemma 12). Due to this, the term $\sum L^{j_{1}} \sigma^{k, j_{2}} I_{j_{1} j_{2}}$ in the Milstein method vanishes. Hence, Euler-Maruyama method is equivalent to Milstein method under normal coordinates. This is one of the reasons we use geodesic instead of straight line as in Dikin walk. We remark that this theorem is used for conveying intuition only.

\subsection{Complex analysis}

A complex function is said to be (complex) analytic (equivalently, holomorphic) if it is locally defined by a convergent power series. Hartog's theorem shows that a complex function in several variables $f: \mathbb{C}^{n} \rightarrow \mathbb{C}$ is holomorphic iff it is analytic in each variable (while fixing all the other variables). For any power series expansion, we define the radius of convergence at $x$ as the largest number $r$ such that the series converges on the sphere with radius $r$ centered at $x$. In this paper, we use the fact that complex analytic functions behave very nicely up to the radius of convergence, and one can avoid complicated and tedious computations by using general convergence theorems.

Theorem 19 (Cauchy's Estimates). Suppose $f$ is holomorphic on a neighborhood of the ball $B \stackrel{\text { def }}{=}$ $\left\{z \in \mathbb{C}:\left|z-z_{0}\right| \leq r\right\}$, then we have that

$$
\left|f^{(k)}\left(z_{0}\right)\right| \leq \frac{k !}{r^{k}} \sup _{z \in B}|f(z)| .
$$

In particular, for any rational function $f(z)=\frac{\prod_{i=1}^{\alpha}\left(z-a_{i}\right)}{\prod_{j=1}^{\beta}\left(z-b_{j}\right)}$ and any ball $B \stackrel{\text { def }}{=}\left\{z \in \mathbb{C}:\left|z-z_{0}\right| \leq r\right\}$ such that $b_{j} \notin B$, we have that

$$
\left|f^{(k)}\left(z_{0}\right)\right| \leq \frac{k !}{r^{k}} \sup _{z \in B}|f(z)|
$$


A similar estimate holds for analytic functions in several variables. We will also use the following classical theorem.

Theorem 20 (Simplified Version of Cauchy-Kowalevski theorem). If $f$ is a complex analytic function defined on a neighborhood of $\left(z_{0}, \alpha\right) \in \mathbb{C}^{n+1}$, then the problem

$$
\frac{d w}{d z}=f(z, w), \quad w\left(z_{0}\right)=\alpha
$$

has a unique complex analytic solution $w$ defined on a neighborhood around $z_{0}$.

Similarly, for a complex analytic function $f$ defined in a neighborhood of $\left(z_{0}, \alpha, \beta\right) \in \mathbb{C}^{2 n+1}$, the $O D E$

$$
\frac{d^{2} w}{d z^{2}}=f\left(z, w, \frac{d w}{d z}\right), \quad w\left(z_{0}\right)=\alpha, \quad \frac{d w}{d z}\left(z_{0}\right)=\beta
$$

has a unique complex analytic solution $w$ defined in a neighborhood around $z_{0}$. 


\section{Convergence of the Geodesic Walk}

The geodesic walk is a Metropolis-filtered Markov chain, whose stationary distribution is the uniform distribution over the polytope to be sampled. We will prove that the conductance of this chain is large with an appropriate choice of the step-size parameter. Therefore, its mixing time to converge to the stationary distribution will be small. The proof of high conductance involves showing (a) the acceptance probability of the Metropolis filter is at least a constant (b) the induced metric satisfies a strong isoperimetric inequality (c) two points that are close in metric distance are also close in probabilistic distance, namely, the one-step distributions from them have large overlap. Besides bounding the number of steps, we also have to show that each step of the Markov chain can be implemented efficiently. We do this in later sections via an efficient algorithm for approximately solving ODEs.

In this section, we present the general conductance bound for Hessian manifolds. The bound on the conductance will use several parameters determined by the specific barrier function. In Section 4 , we bound these parameters for the logarithmic barrier.

For a Markov chain with state space $M$, stationary distribution $Q$ and next step distribution $P_{u}(\cdot)$ for any $u \in M$, the conductance of the Markov chain is

$$
\phi=\inf _{S \subset M} \frac{\int_{S} P_{u}(M \backslash S) d Q(u)}{\min \{Q(S), Q(M \backslash S)\}} .
$$

The conductance of an ergodic Markov chain allows us to bound its mixing time, i.e., the rate of convergence to its stationary distribution, e.g., via the following theorem of Lovász and Simonovits.

Theorem 21 ([24]). Let $Q_{t}$ be the distribution of the current point after $t$ steps of a Markov chain with stationary distribution $Q$ and conductance at least $\phi$, starting from initial distribution $Q_{0}$. Then,

$$
d_{T V}\left(Q_{t}, Q\right) \leq \sqrt{d_{0}}\left(1-\frac{\phi^{2}}{2}\right)^{t}
$$

where $d_{0}=\mathbb{E}_{Q_{0}}\left(d Q_{0}(u) / d Q(u)\right)$ is a measure of the distance of the starting distribution from the stationary and $d_{T V}$ is the total variation distance.

\subsection{Hessian parameters}

The mixing of the walk depends on the maximum values of several smoothness parameters of the manifold. Since each step of our walk involves a Gaussian vector which can be large with some probability, many smoothness parameters inevitably depend on this Gaussian vector. Formally, let $\gamma$ be the geodesic used in a step of the geodesic walk with the parameterization $\gamma:[0, \ell] \rightarrow M$ where $\ell \stackrel{\text { def }}{=} \sqrt{n h}$. Note that $\ell$ is not exactly the length of the geodesic step, but it is close with high probability due to Gaussian concentration. Rather than using supremum bounds for our smoothness parameters, it suffices to use large probability bounds, where the probability is over the choice of geodesic at any point $x \in \Omega$. To capture this notion that "most geodesics are good", we allow the use of an auxiliary function $V(\gamma) \geq 0$ to measure how good a geodesic is. Several of the smoothness parameters assume that this function is bounded and Lipshitz for a sufficiently large step size $h$. More precisely, viewing geodesics as maps $\gamma:[0, \ell] \rightarrow M$, we assume that there exists an auxiliary real function on the tangent bundle (union of tangent manifolds for all points in $M$ ), $V: T M \rightarrow \mathbb{R}_{+}$, satisfying 
1. For $h \leq H$ and any variation of geodesics $\gamma_{s}$ with $V\left(\gamma_{s}\right) \leq V_{0}$, there is a $V_{1} \geq V_{0}$ s.t. $\left|\frac{d}{d s} V\left(\gamma_{s}\right)\right| \leq V_{1}\left(\left\|\frac{d}{d s} \gamma_{s}(0)\right\|_{\gamma_{s}(0)}+\ell\left\|D_{s} \gamma_{s}\right\|_{\gamma_{s}(0)}\right)$

2. For any $x \in M$,

$$
\mathbb{P}_{\text {geodesic } \gamma \text { from } x}\left(V(\gamma) \leq \frac{1}{2} V_{0}\right) \geq 1-\frac{V_{0}}{100 V_{1}}
$$

Definition 22. Given a Hessian manifold $M$, maximum step size $H$ and auxiliary function $V$ with parameters $V_{0}, V_{1}$, we define the smoothness parameters $D_{0}, D_{1}, D_{2}, G_{1}, G_{2}, R_{1}, R_{2}$ depending only on $M$ and the step size $h \leq H$ as follows:

1. The maximum norm of the drift in the local metric, $D_{0}=\sup _{x \in M}\|\mu(x)\|_{x}$.

2. The smoothness of the norm of the drift, $D_{1}=\sup _{h \leq H, V(\gamma) \leq V_{0}, 0 \leq t \leq \ell} \frac{d}{d t}\|\mu(\gamma(t))\|_{\gamma(t)}^{2}$.

3. The smoothness of the drift, $D_{2}=\sup _{x \in M,\|s\|_{x} \leq 1}\left\|\nabla_{s} \mu(x)\right\|_{x}$.

4. The smoothness of the local volume, $\left.G_{1}=\sup _{h \leq H, V(\gamma) \leq V_{0}, 0 \leq t \leq \ell} \mid \log \operatorname{det}(g(\gamma(t)))\right)^{\prime \prime \prime} \mid$ where $g(x)$ is the metric at $x$.

5. The smoothness of the metric, $G_{2}=\sup \frac{d(x, y)}{d_{H}(x, y)}$ where $d_{H}$ is the Hilbert distance (defined in Section 3.2) and $d$ is the shortest path distance in $M$.

6. The stability of the Jacobian field, $R_{1}=\sup _{h \leq H, V(\gamma) \leq V_{0}, 0 \leq t \leq \ell}\|R(t)\|_{F}$ where $R(t)$ is defined in Definition 8.

7. The smoothness of the Ricci curvature, $R_{2}=\sup _{h \leq H, V(\gamma) \leq V_{0}}\left|\frac{d}{d s} \operatorname{Ric}\left(\gamma_{s}^{\prime}(t)\right)\right|$ (see Definition $37)$.

We refer to these as the parameters of a Hessian manifold. Our main theorem for convergence can be stated as follows.

Theorem 23. On a Hessian manifold of dimension $n$ with an auxiliary function, step-size upper bound $H$ and parameters $D_{0}, D_{1}, D_{2}, G_{1}, G_{2}, R_{1}, R_{2}$, the geodesic walk with step size

$$
h \leq \Theta(1) \min \left\{\frac{1}{n^{1 / 3} D_{1}^{2 / 3}}, \frac{1}{D_{2}}, \frac{1}{n R_{1}}, \frac{1}{\left(n D_{0} R_{1}\right)^{2 / 3}}, \frac{1}{\left(n R_{2}\right)^{2 / 3}}, \frac{1}{n G_{1}^{2 / 3}}, H\right\}
$$

has conductance $\Omega\left(\sqrt{h} / G_{2}\right)$ and mixing time $O\left(G_{2}^{2} / h\right)$.

In the rest of this section, we prove this theorem. It can be sepecialized to any Hessian manifold by bounding the parameters. In later sections, we do this for the log barrier, by defining the auxiliary function and bounding the manifold parameters.

\subsection{Isoperimetry}

For a convex body $K$, the cross-ratio distance of $x$ and $y$ is

$$
d_{K}(x, y)=\frac{|x-y||p-q|}{|p-x||y-q|}
$$

where $p$ and $q$ are on the boundary of $K$ such that $p, x, y, q$ are on the straight line $\overline{x y}$ and are in order. In this section, we show that if the distance $d(x, y)$ induced by the Riemannian metric is 
upper bounded by the cross-ratio distance, then the body has good isoperimetric constant in terms of $d$. We note that although the cross-ratio distance is not a metric, the closely-related Hilbert distance is a metric:

$$
d_{H}(x, y)=\log \left(1+\frac{|x-y||p-q|}{|p-x||y-q|}\right) .
$$

Theorem 24. For a Hessian manifold $M$ with smoothness parameters $G_{2}$, for any partition of $M$ into three measurable subsets $S_{1}, S_{2}, S_{3}$, we have that

$$
\operatorname{vol}\left(S_{3}\right) \geq \frac{d\left(S_{1}, S_{2}\right)}{G_{2}} \min \left\{\operatorname{vol}\left(S_{1}\right), \operatorname{vol}\left(S_{2}\right)\right\} .
$$

The theorem follows from the following isoperimetric inequality from [21], the definition of $G_{2}$ and the fact $d_{H} \leq d_{K}$.

Theorem 25 ([21]). For any convex body $K$ and any partition of $K$ into disjoint measurable subsets $S_{1}, S_{2}, S_{3}$

$$
\operatorname{vol}\left(S_{3}\right) \geq d_{K}\left(S_{1}, S_{2}\right) \operatorname{vol}\left(S_{1}\right) \operatorname{vol}\left(S_{2}\right) .
$$

\subsection{1-step distribution}

We first derive a formula for the drift term - it is in fact a classical Newton step of the volumetric barrier function $\log \operatorname{det} \nabla^{2} \phi(x)$.

Lemma 26. We have

$$
\mu(x)=-\left(\nabla^{2} \phi(x)\right)^{-1} \nabla \psi(x)
$$

where $\psi(x)=\frac{1}{2} \log \operatorname{det} \nabla^{2} \phi(x)$.

Proof. We note that

$$
\begin{aligned}
\frac{\partial}{\partial x_{j}} \log \operatorname{det}\left(\nabla^{2} \phi\right)^{-1} & =\operatorname{Tr}\left(\left(\nabla^{2} \phi\right) \frac{\partial}{\partial x_{j}}\left(\nabla^{2} \phi\right)^{-1}\right) \\
& =-\operatorname{Tr}\left(\left(\nabla^{2} \phi\right)\left(\nabla^{2} \phi\right)^{-1}\left(\frac{\partial}{\partial x_{j}} \nabla^{2} \phi\right)\left(\nabla^{2} \phi\right)^{-1}\right) \\
& =-\sum_{k} e_{k}^{T}\left(\frac{\partial}{\partial x_{j}} \nabla^{2} \phi\right)\left(\nabla^{2} \phi\right)^{-1} e_{k} .
\end{aligned}
$$

Hence, we have

$$
\begin{aligned}
\frac{1}{2} e_{i}^{T}\left(\nabla^{2} \phi\right)^{-1} \nabla \log \operatorname{det}\left(\nabla^{2} \phi\right)^{-1} & =-\frac{1}{2} \sum_{j k}\left(\nabla^{2} \phi\right)_{i j}^{-1} e_{k}^{T}\left(\frac{\partial}{\partial x_{j}} \nabla^{2} \phi\right)\left(\nabla^{2} \phi\right)^{-1} e_{k} \\
& =-\frac{1}{2} \sum_{j k} e_{i}^{T}\left(\nabla^{2} \phi\right)^{-1}\left(\frac{\partial}{\partial x_{k}} \nabla^{2} \phi\right)\left(\nabla^{2} \phi\right)^{-1} e_{k}
\end{aligned}
$$

On the other hand, we have

$$
\begin{aligned}
\mu_{i} & =\frac{1}{2} \sum_{k} \frac{\partial}{\partial x_{k}}\left(\left(\nabla^{2} \phi\right)^{-1}\right)_{i k} \\
& =-\frac{1}{2} \sum_{k} e_{i}^{T}\left(\nabla^{2} \phi\right)^{-1}\left(\frac{\partial}{\partial x_{k}} \nabla^{2} \phi\right)\left(\nabla^{2} \phi\right)^{-1} e_{k} .
\end{aligned}
$$


To have a uniform stationary distribution, the geodesic walk uses a Metropolis filter. The transition probability before applying the filter is given as follows in Euclidean coordinates.

Lemma 27. For any $x \in M$ and $h>0$, the probability density of the 1-step distribution from $x$ (before applying the Metropolis filter) is given by

$$
p_{x}(y)=\sum_{v_{x}: \exp _{x}\left(v_{x}\right)=y} \operatorname{det}\left(d \exp _{x}\left(v_{x}\right)\right)^{-1} \sqrt{\frac{\operatorname{det}(g(y))}{(2 \pi h)^{n}}} \exp \left(-\frac{1}{2}\left\|\frac{v_{x}-\frac{h}{2} \mu(x)}{\sqrt{h}}\right\|_{x}^{2}\right)
$$

where $y=\exp _{x}\left(v_{x}\right)$ and $d \exp _{x}$ is the differential of the exponential map at $x$.

Proof. We prove the formula by separately considering each $v_{x} \in T_{x} M$ s.t. $\exp _{x}\left(v_{x}\right)=y$, then summing up. In the tangent space $T_{x} M$, the point $v_{x}$ is a Gaussian step. Therefore, the probability density of $v_{x}$ in $T_{x} M$ as follows.

$$
p_{x}^{T_{x} M}\left(v_{x}\right)=\frac{1}{(2 \pi h)^{n / 2}} \exp \left(-\frac{1}{2}\left\|\frac{v_{x}-\frac{h}{2} \mu(x)}{\sqrt{h}}\right\|_{x}^{2}\right) .
$$

Note that $v_{x}, \mu(x) \in T_{x} M$. Let $y=\exp _{x}\left(v_{x}\right)$. In the tangent space $T_{y} M$, we have that $y$ maps to 0 . Let $F: T_{x} M \rightarrow K$ defined by $F(v)=\operatorname{id}_{M \rightarrow K} \circ \exp _{x}(v)$. Here $K$ is the same set as $M$ but endowed with the Euclidean metric. Hence, we have

$$
d F\left(v_{x}\right)=\operatorname{did}_{M \rightarrow K}(y) d \exp _{x}\left(v_{x}\right) .
$$

The result follows from $p_{x}(y)=\operatorname{det}\left(d F\left(v_{x}\right)\right)^{-1} p_{x}^{T_{x} M}\left(v_{x}\right)$ and

$$
\begin{aligned}
\operatorname{det} d F\left(v_{x}\right) & =\operatorname{det}\left(\operatorname{did}_{M \rightarrow K}(y)\right) \operatorname{det}\left(d \exp _{x}\left(v_{x}\right)\right) \\
& =\operatorname{det}(g(y))^{-1 / 2} \operatorname{det}\left(d \exp _{x}\left(v_{x}\right)\right)
\end{aligned}
$$

In Section 3.6, we bound the acceptance probability of the Metropolis filter. This is a crucial aspect of the analysis.

Theorem 28. Given a geodesic $\gamma$ with $\gamma(0)=x, \gamma^{\prime}(0)=v_{x}, \gamma(\ell)=y, \gamma^{\prime}(\ell)=-v_{y}$ with $\ell=\sqrt{n h}$. Suppose that $h \leq \min \left(H, \frac{1}{n R_{1}}\right)$ and $V(\gamma) \leq V_{0}$, then we have that

$$
\left|\log \left(\frac{p\left(x \stackrel{v_{x}}{\rightarrow} y\right)}{p\left(y \stackrel{v_{y}}{\rightarrow} x\right)}\right)\right|=O\left(\sqrt{n} h^{3 / 2} D_{1}+(n h)^{3 / 2} G_{1}+\left(n h R_{1}\right)^{2}\right) .
$$

In Section 3.7, we bound the overlap of one-step distributions from nearby points.

Theorem 29. For $h \leq \min \left(H, \frac{1}{10^{6} n R_{1}}\right)$, then the one-step distributions $P_{x}, P_{z}$ from $x, z$ satisfy

$$
d_{T V}\left(P_{x}, P_{z}\right)=O\left(n h R_{2}+D_{2} \sqrt{h}+\frac{1}{\sqrt{h}}+n h D_{0} R_{1}\right) d(x, z)+\frac{1}{20} .
$$

Combining the above two theorems lets us bound the conductance and mixing time of the walk, as we show in the next section. 


\subsection{Conductance and mixing time}

Proof of Theorem 23. The proof follows the standard outline for geometric random walks (see e.g., [36]). Let $Q$ be the uniform distribution over $M$ and $S$ be any measurable subset of $M$. Then our goal is to show that

$$
\frac{\int_{S} P_{x}(M \backslash S) d Q(x)}{\min \{Q(S), Q(M \backslash S)\}}=\Omega\left(\frac{\sqrt{h}}{G_{2}}\right) .
$$

Since the Markov chain is time-reversible (For any two subsets $A, B, \int_{A} P_{x}(B) d x=\int_{B} P_{x}(A) d x$ ), we can write the numerator of the LHS above as

$$
\frac{1}{2}\left(\int_{S} P_{x}(M \backslash S) d Q(x)+\int_{M \backslash S} P_{x}(S) d Q(x)\right) .
$$

Define

$$
\begin{aligned}
& S_{1}=\left\{x \in S: P_{x}(M \backslash S)<0.05\right\} \\
& S_{2}=\left\{x \in M \backslash S: P_{x}(S)<0.05\right\} \\
& S_{3}=M \backslash S_{1} \backslash S_{2} .
\end{aligned}
$$

We can assume wlog that $Q\left(S_{1}\right) \geq(1 / 2) Q(S)$ and $Q\left(S_{2}\right) \geq(1 / 2) Q(M \backslash S)$ (if not, the conductance is $\Omega(1)$ ).

Next, we note that for any two points $x \in S_{1}$ and $y \in S_{2}, d_{T V}\left(P_{x}, P_{y}\right)>0.9$. Therefore, by Theorem 25, we have that $d(x, y)=\Omega(\sqrt{h})$ and hence $d\left(S_{1}, S_{2}\right)=\Omega(\sqrt{h})$. Therefore, using Theorem 24,

$$
\operatorname{vol}\left(S_{3}\right)=\Omega\left(\frac{\sqrt{h}}{G_{2}}\right) \min \left\{\operatorname{vol}\left(S_{1}\right), \operatorname{vol}\left(S_{2}\right)\right\} .
$$

Going back to the conductance,

$$
\begin{aligned}
\frac{1}{2}\left(\int_{S} P_{x}(M \backslash S) d Q(x)+\int_{M \backslash S} P_{x}(S) d Q(x)\right) & \geq \frac{1}{2} \int_{S_{3}}(0.05) d Q(x) \\
& =\Omega\left(\frac{\sqrt{h}}{G_{2}}\right) \min \left\{\operatorname{vol}\left(S_{1}\right), \operatorname{vol}\left(S_{2}\right)\right\} \frac{1}{\operatorname{vol}(M)} \\
& =\Omega\left(\frac{\sqrt{h}}{G_{2}}\right) \min \left\{\frac{\operatorname{vol}(S)}{\operatorname{vol}(M)}, \frac{\operatorname{vol}(M \backslash S)}{\operatorname{vol}(M)}\right\} \\
& =\Omega\left(\frac{\sqrt{h}}{G_{2}}\right) \min \{Q(S), Q(M \backslash S)\}
\end{aligned}
$$

Therefore, $\phi(S) \geq \Omega\left(\frac{\sqrt{h}}{G_{2}}\right)$.

Corollary 30. Let $K$ be a polytope. Let $Q$ be the uniform distribution over $K$ and $Q_{t}$ be the distribution obtained after $t$ steps of the geodesic walk started from a distribution $Q_{0}$ with $d_{0}=\sup _{K} \frac{d Q_{0}}{d Q}$. Then after $t>O\left(G_{2}^{2} / h\right) \log \left(\frac{d_{0}}{\epsilon}\right)$ steps, with probability at least $1-\delta$, we have $d_{T V}\left(Q_{t}, Q\right) \leq \epsilon$. 


\subsection{Warm-up: Interval and hypercube}

The technical core of the proof is in Theorems 28 and 29. Before we get to those, in this section, we analyze the geodesic walk for one-dimensional Hessian manifolds and the hypercube (product of intervals), which we can do by more elementary methods.

Lemma 31. Given a barrier function $\phi$ on one dimension interval $(\alpha, \beta)$ such that $\left|\phi^{(3)}(x)\right|=$ $O\left(\phi^{\prime \prime}(x)^{3 / 2}\right),\left|\phi^{(4)}(x)\right|=O\left(\phi^{\prime \prime}(x)^{4 / 2}\right)$ and $\left|\phi^{(5)}(x)\right|=O\left(\phi^{\prime \prime}(x)^{5 / 2}\right)$ for $x \in(\alpha, \beta)$. For step size $h$ smaller than some constant, the geodesic walk on the Hessian manifold $(\alpha, \beta)$ induced by $\phi$ satisfies that

$$
\left|\log \left(\frac{p(x \rightarrow y)}{p(y \rightarrow x)}\right)\right|=O\left(h^{3 / 2}\right)
$$

with constant probability.

The first assumption above $\left|\phi^{(3)}(x)\right|=O\left(\phi^{\prime \prime}(x)^{3 / 2}\right)$ is called self-concordance, and the others can be viewed as its extensions to higher derivatives. It is easy to check that the logarithmic barrier $\phi(x)=-\log (1+x)-\log (1-x)$ on $(-1,1)$ satisfies all the assumptions. Our main theorem for general manifolds (Theorem 28) implies the same bound after substituting the parameters for the $\log$ barrier on the interval with $n=1$ and $m=2$.

Proof. Since the walk is affine-invariant and the conditions are scale-invariant, we can assume the domain is the interval $(-1,1)$. Let the metric $p(x)=\phi^{\prime \prime}(x)$. The drift is given by $(1.2)$ :

$$
\mu(x)=\frac{1}{2} \frac{d}{d x}\left(\frac{1}{p(x)}\right)=\frac{-p^{\prime}(x)}{2 p^{2}(x)} .
$$

Let $f(x) \stackrel{\text { def }}{=} \int_{0}^{x} \sqrt{p(t)} d t$ be the mapping from $(-1,1)$ to $\mathbb{R}$ such that for any $x, y \in(-1,1)$, we have that $|f(x)-f(y)|$ is the manifold distance on $(-1,1)$ using the metric $p$. In particular, we have that

$$
\exp _{x}(v)=f^{-1}\left(f(x)+p^{1 / 2}(x) v\right) .
$$

Therefore, the probability density on $\mathbb{R}$ (as in Lemma 27 for the one-dimensional case) is given by

$$
p^{\mathbb{R}}(x \rightarrow y)=\frac{1}{\sqrt{2 \pi h}} \exp \left[-\frac{1}{2 h}\left(f(x)-\frac{h p^{\prime}(x)}{4 p^{3 / 2}(x)}-f(y)\right)^{2}\right] .
$$

Hence,

$$
p(x \rightarrow y)=\sqrt{\frac{p(y)}{2 \pi h}} \exp \left[-\frac{1}{2 h}\left(f(x)-\frac{h p^{\prime}(x)}{4 p^{3 / 2}(x)}-f(y)\right)^{2}\right] .
$$

Hence, we have that

$$
\begin{aligned}
& \frac{p(x \rightarrow y)}{p(y \rightarrow x)} \\
= & \sqrt{\frac{p(y)}{p(x)}} \exp \left[\frac{1}{2 h}\left(f(y)-f(x)-\frac{h p^{\prime}(y)}{4 p^{3 / 2}(y)}\right)^{2}-\frac{1}{2 h}\left(f(y)-f(x)+\frac{h p^{\prime}(x)}{4 p^{3 / 2}(x)}\right)^{2}\right] \\
= & \exp \left[\frac{\log (p(y))-\log (p(x))}{2}-\frac{f(y)-f(x)}{4}\left(\frac{p^{\prime}(x)}{p^{3 / 2}(x)}+\frac{p^{\prime}(y)}{p^{3 / 2}(y)}\right)+\frac{h}{32}\left(\frac{\left(p^{\prime}(y)\right)^{2}}{p^{3}(y)}-\frac{\left(p^{\prime}(x)\right)^{2}}{p^{3}(x)}\right)\right] .
\end{aligned}
$$


Note that

$$
\begin{aligned}
\log (p(y))-\log (p(x)) & =\log \left(p\left(f^{-1}(f(y))\right)\right)-\log \left(p\left(f^{-1}(f(x))\right)\right) \\
& =\int_{f(x)}^{f(y)} \frac{p^{\prime}\left(f^{-1}(t)\right)}{p\left(f^{-1}(t)\right) f^{\prime}\left(f^{-1}(t)\right)} d t \\
& =\int_{f(x)}^{f(y)} \frac{p^{\prime}\left(f^{-1}(t)\right)}{p^{3 / 2}\left(f^{-1}(t)\right)} d t
\end{aligned}
$$

Note that for any second differentiable function $\phi$, Lemma 32 shows that that

$$
\left|\int_{\beta}^{\alpha} \phi(t) d t-(\alpha-\beta)(\phi(\alpha)+\phi(\beta))\right| \leq \frac{|\alpha-\beta|^{3}}{12} \max _{\alpha \leq t \leq \beta}\left|\phi^{\prime \prime}(t)\right| .
$$

Hence,

$$
\begin{aligned}
& \left|\log (p(y))-\log (p(x))-\frac{f(y)-f(x)}{2}\left(\frac{p^{\prime}(x)}{p^{3 / 2}(x)}+\frac{p^{\prime}(y)}{p^{3 / 2}(y)}\right)\right| \\
\leq & \frac{|f(x)-f(y)|^{3}}{12} \max _{f(x) \leq t \leq f(y)}\left|\frac{d^{2}}{d t^{2}} \frac{p^{\prime}\left(f^{-1}(t)\right)}{p^{3 / 2}\left(f^{-1}(t)\right)}\right| .
\end{aligned}
$$

For the other term, we note that

$$
\left|\frac{\left(p^{\prime}(y)\right)^{2}}{p^{3}(y)}-\frac{\left(p^{\prime}(x)\right)^{2}}{p^{3}(x)}\right| \leq|y-x| \max _{x \leq t \leq y}\left|\frac{d}{d t} \frac{\left(p^{\prime}(t)\right)^{2}}{p^{3}(t)}\right| .
$$

Hence,

$$
\begin{aligned}
& \left|\log \left(\frac{p(x \rightarrow y)}{p(y \rightarrow x)}\right)\right| \\
\leq & O\left(|f(x)-f(y)|^{3} \max _{f(x) \leq t \leq f(y)}\left|\frac{d^{2}}{d t^{2}} \frac{p^{\prime}\left(f^{-1}(t)\right)}{p^{3 / 2}\left(f^{-1}(t)\right)}\right|\right)+O\left(h|y-x| \max _{x \leq t \leq y}\left|\frac{d}{d t} \frac{\left(p^{\prime}(t)\right)^{2}}{p^{3}(t)}\right|\right) .
\end{aligned}
$$

Note that

$$
\frac{d}{d t} \frac{p^{\prime}\left(f^{-1}(t)\right)}{p^{3 / 2}\left(f^{-1}(t)\right)}=-\frac{3}{2} \frac{\left(p^{\prime}\left(f^{-1}(t)\right)\right)^{2}}{p^{3}\left(f^{-1}(t)\right)}+\frac{p^{\prime \prime}\left(f^{-1}(t)\right)}{p^{2}\left(f^{-1}(t)\right)}
$$

and

$$
\frac{d^{2}}{d t^{2}} \frac{p^{\prime}\left(f^{-1}(t)\right)}{p^{3 / 2}\left(f^{-1}(t)\right)}=\frac{9}{2} \frac{\left(p^{\prime}\left(f^{-1}(t)\right)\right)^{3}}{p^{9 / 2}\left(f^{-1}(t)\right)}-\frac{3 p^{\prime}\left(f^{-1}(t)\right) p^{\prime \prime}\left(f^{-1}(t)\right)}{p^{7 / 2}\left(f^{-1}(t)\right)}+\frac{p^{\prime \prime \prime}\left(f^{-1}(t)\right)}{p^{5 / 2}\left(f^{-1}(t)\right)} .
$$

Also, we have that

$$
\frac{d}{d t} \frac{\left(p^{\prime}(t)\right)^{2}}{p^{3}(t)}=\frac{2 p^{\prime}(t) p^{\prime \prime}(t)}{p^{3}(t)}-\frac{3\left(p^{\prime}(t)\right)^{3}}{p^{4}(t)}
$$

Hence, we have that

$$
\begin{aligned}
\left|\log \left(\frac{p(x \rightarrow y)}{p(y \rightarrow x)}\right)\right|= & O\left(|f(x)-f(y)|^{3} \max _{x \leq t \leq y}\left(\left|\frac{\left(p^{\prime}(t)\right)^{3}}{p^{9 / 2}(t)}\right|+\left|\frac{p^{\prime}(t) p^{\prime \prime}(t)}{p^{7 / 2}(t)}\right|+\left|\frac{p^{\prime \prime \prime}(t)}{p^{5 / 2}(t)}\right|\right)\right) \\
& +O\left(h|y-x| \max _{x \leq t \leq y}\left(\left|\frac{p^{\prime}(t) p^{\prime \prime}(t)}{p^{3}(t)}\right|+\left|\frac{\left(p^{\prime}(t)\right)^{3}}{p^{4}(t)}\right|\right)\right) .
\end{aligned}
$$


Since $\phi$ is self-concordant, i.e., $\left|\phi^{(3)}(x)\right|=O\left(\phi^{\prime \prime}(x)^{3 / 2}\right)$, we know that $p(t) \leq O(1) p(s)$ for all $s, t \in$ $[x, y]$ if $p(x)|x-y|$ is smaller than a constant. Noting that $p(x)|x-y|^{2}=\Theta(h)$ with constant probability, we have that $p(t) \leq O(1) p(s)$ for all $s, t \in[x, y]$. Therefore, we have that

$$
\begin{aligned}
|f(x)-f(y)| & =O(|x-y| \sqrt{p(x)})=O(\sqrt{h}), \\
|x-y| & =O\left(p^{-1 / 2}(x) \sqrt{h}\right) .
\end{aligned}
$$

Hence, we have

$$
\begin{aligned}
& \left|\log \left(\frac{p(x \rightarrow y)}{p(y \rightarrow x)}\right)\right| \\
= & O\left(h^{3 / 2} \max _{x \leq t \leq y}\left(\left|\frac{\left(p^{\prime}(t)\right)^{3}}{p^{9 / 2}(t)}\right|+\left|\frac{p^{\prime}(t) p^{\prime \prime}(t)}{p^{7 / 2}(t)}\right|+\left|\frac{p^{\prime \prime \prime}(t)}{p^{5 / 2}(t)}\right|\right)\right)+O\left(h^{3 / 2} \max _{x \leq t \leq y}\left(\left|\frac{p^{\prime}(t) p^{\prime \prime}(t)}{p^{7 / 2}(t)}\right|+\left|\frac{\left(p^{\prime}(t)\right)^{3}}{p^{9 / 2}(t)}\right|\right)\right) .
\end{aligned}
$$

Using our assumption on $\phi$, we get our result.

Remark. The hypercube in $\mathbb{R}^{n}$ is a product of intervals. In fact, the next-step density function is a product function, and the quantity $\log \left(\frac{p(x \rightarrow y)}{p(y \rightarrow x)}\right)$ is a sum over the same quantity in each coordinate. Viewing the coordinates as independent processes, this is a sum of independent random variables, w.h.p. of magnitude $O\left(h^{\frac{3}{2}}\right)$. The signs are random since ratio is symmetric in $x, y$. Thus, the overall $\log$ ratio is $O\left(\sqrt{n} h^{\frac{3}{2}}\right)$. To keep this bounded, it suffices to choose $h=O\left(n^{-\frac{1}{3}}\right)$. Moreover, since the isoperimetric ratio $\left(G_{2}\right.$ in our parametrization) is $O(1)$ for the hypercube, this gives an overall mixing time of $O\left(n^{\frac{1}{3}}\right)$, much lower than the mixing time of $n^{2}$ for the ball walk and hit-and-run, or the current bound for the Dikin walk.

\subsection{Rejection probability}

The goal of this section is to prove Theorem 28, i.e., the rejection probability of the Metropolis filter is small. For a transition from $x$ to $y$ on the manifold, the filter is applied with respect to a randomly chosen $v_{x}$, i.e., for one geodesic from $x$ to $y$. We will bound the ratio of the transition probabilities (without the filter) as follows:

$$
\begin{aligned}
\log \left(\frac{p\left(x \stackrel{v_{x}}{\rightarrow} y\right)}{p\left(y \stackrel{v_{y}}{\rightarrow} x\right)}\right)= & \log \left(\frac{\operatorname{det}\left(d \exp _{x}\left(v_{x}\right)\right)^{-1}}{\operatorname{det}\left(d \exp _{y}\left(v_{y}\right)\right)^{-1}}\right)+\frac{1}{2} \log \operatorname{det}(g(y))-\frac{1}{2} \log \operatorname{det}(g(x)) \\
& -\frac{1}{2}\left\|\frac{v_{x}-\frac{h}{2} \mu(x)}{\sqrt{h}}\right\|_{x}^{2}+\frac{1}{2}\left\|\frac{v_{y}-\frac{h}{2} \mu(y)}{\sqrt{h}}\right\|_{y}^{2} .
\end{aligned}
$$

Since a geodesic has constant speed, we have $\left\|v_{x}\right\|_{x}=\left\|v_{y}\right\|_{y}$. Therefore, we have that

$$
\begin{aligned}
\log \left(\frac{p\left(x \stackrel{v_{x}}{\rightarrow} y\right)}{p\left(y \stackrel{v_{y}}{\rightarrow} x\right)}\right)= & \log \left(\frac{\operatorname{det}\left(d \exp _{y}\left(v_{y}\right)\right)}{\operatorname{det}\left(d \exp _{x}\left(v_{x}\right)\right)}\right)+\frac{1}{2} \log \operatorname{det}(g(y))-\frac{1}{2} \log \operatorname{det}(g(x)) \\
& +\frac{1}{2}\left\langle v_{x}, \mu(x)\right\rangle_{x}-\frac{h}{8}\|\mu(x)\|_{x}^{2}-\frac{1}{2}\left\langle v_{y}, \mu(y)\right\rangle_{y}+\frac{h}{8}\|\mu(y)\|_{y}^{2} .
\end{aligned}
$$

We separate the proof into three parts:

- $\left|\|\mu(x)\|_{x}^{2}-\|\mu(y)\|_{y}^{2}\right| \leq \sqrt{n h} D_{1}$, immediate from the definition of $D_{1}$ and the parameterization $\gamma:[0, \ell \stackrel{\text { def }}{=} \sqrt{n h}] \rightarrow M$. 
- $\operatorname{Sec} 3.6 .1:\left|\log \operatorname{det}(g(y))-\log \operatorname{det}(g(x))+\left\langle v_{x}, \mu(x)\right\rangle_{x}-\left\langle v_{y}, \mu(y)\right\rangle_{y}\right|=O\left((n h)^{3 / 2} G_{1}\right)$,

- Sec 3.6.2: $\left|\log \left(\frac{\operatorname{det}\left(d \exp _{y}\left(v_{y}\right)\right)}{\operatorname{det}\left(d \exp _{x}\left(v_{x}\right)\right)}\right)\right|=O\left(\left(n h R_{1}\right)^{2}\right)$.

Together, these facts imply Theorem 28.

\subsubsection{Trapezoidal rule}

Recall that the trapezoidal rule is to approximate $\int_{0}^{h} f(t) d t$ by $\frac{h}{2}(f(0)+f(h))$. The nice thing about this rule is that the error is $O\left(h^{3}\right)$ instead of $O\left(h^{2}\right)$ because the second order term cancels by symmetry. Our main observation here is that the geodesic walk implicitly follows a trapezoidal rule on the metric and hence it has a small error. We include the proof of the trapezoidal rule error for completeness.

Lemma 32. We have that

$$
\left|\int_{0}^{\ell} f(t) d t-\frac{\ell}{2}(f(0)+f(\ell))\right| \leq \frac{\ell^{3}}{12} \max _{0 \leq t \leq \ell}\left|f^{\prime \prime}(t)\right| .
$$

Proof. Note that

$$
\begin{aligned}
\int_{0}^{\ell} f(t) d t-\frac{\ell}{2}(f(0)+f(\ell)) & =\int_{0}^{\ell}\left(f(0)+\int_{0}^{t} f^{\prime}(s) d s\right) d t-\ell f(0)-\frac{\ell}{2} \int_{0}^{\ell} f^{\prime}(s) d s \\
& =\int_{0}^{\ell} \int_{0}^{t} f^{\prime}(s) d s d t-\frac{\ell}{2} \int_{0}^{\ell} f^{\prime}(s) d s \\
& =\int_{0}^{\ell}\left(\frac{\ell}{2}-s\right) f^{\prime}(s) d s \\
& =\int_{0}^{\ell}\left(\frac{\ell}{2}-s\right)\left(f^{\prime}\left(\frac{\ell}{2}\right)+\int_{\ell / 2}^{s} f^{\prime \prime}(t) d t\right) d s \\
& =\int_{0}^{\ell}\left(\frac{\ell}{2}-s\right) \int_{\ell / 2}^{s} f^{\prime \prime}(t) d t d s \\
& \leq \frac{\ell^{3}}{12} \max _{0 \leq t \leq \ell}\left|f^{\prime \prime}(t)\right| .
\end{aligned}
$$

We apply this to the logdet function.

Lemma 33. Let $f(t)=\log \operatorname{det}\left(g(\gamma(t))\right.$ where $\gamma(t)=\exp _{x}\left(\frac{t}{\ell} v_{x}\right)$. If $h \leq H$ and $V(\gamma) \leq V_{0}$, we have

$$
\left|\log \operatorname{det}(g(y))-\log \operatorname{det}(g(x))+\left\langle v_{x}, \mu(x)\right\rangle_{x}-\left\langle v_{y}, \mu(y)\right\rangle_{y}\right|=O\left((n h)^{3 / 2} G_{1}\right) .
$$

Proof. Let $f(t)=\log \operatorname{det} g(\gamma(t))$. By Lemma 26, $\mu(\gamma(t))=-\frac{1}{2} g(\gamma(t))^{-1} \nabla f(\gamma(t))$. Using this,

$$
\begin{aligned}
f^{\prime}(t) & =\left\langle\nabla_{\gamma(t)} \log \operatorname{det} g(\gamma(t)), \gamma^{\prime}(t)\right\rangle_{2} \\
& =\left\langle g(\gamma(t))^{-1} \nabla_{\gamma(t)} \log \operatorname{det} g(\gamma(t)), \gamma^{\prime}(t)\right\rangle_{\gamma(t)} \\
& =-\left\langle 2 \mu(\gamma(t)), \gamma^{\prime}(t)\right\rangle_{\gamma(t)} .
\end{aligned}
$$


Noting that $v_{x}=\ell \gamma^{\prime}(0)$ and $v_{y}=-\ell \gamma^{\prime}(\ell)$, and using Lemma 32, we have

$$
\begin{aligned}
& \left|\log \operatorname{det}(g(y))-\log \operatorname{det}(g(x))+\left\langle v_{x}, \mu(x)\right\rangle_{x}-\left\langle v_{y}, \mu(y)\right\rangle_{y}\right| \\
= & \left|\log \operatorname{det}(g(y))-\log \operatorname{det}(g(x))+\ell\left(\left\langle\gamma^{\prime}(0), \mu(x)\right\rangle_{x}+\left\langle\gamma^{\prime}(\ell), \mu(y)\right\rangle_{y}\right)\right| \\
= & \left|\int_{0}^{\ell} f^{\prime}(t) d t-\frac{\ell}{2}\left(f^{\prime}(0)+f^{\prime}(\ell)\right)\right| \\
\leq & \frac{\ell^{3}}{12} \max _{0 \leq t \leq \ell}\left|f^{\prime \prime \prime}(t)\right|=O\left((n h)^{3 / 2} G_{1}\right) .
\end{aligned}
$$

\subsubsection{Smoothness of exponential map}

First, we show the relation between the differential of exponential map $d \exp _{x}\left(v_{x}\right)$ and the Jacobi field along the geodesic $\exp _{x}\left(t v_{x}\right)$. This can be viewed as the fundamental connection between geodesics and the Jacobi field in matrix notation, which will be convenient for our purpose.

Lemma 34. Given a geodesic $\gamma(t)=\exp _{x}\left(\frac{t}{\ell} v_{x}\right)$, let $\left\{X_{i}(t)\right\}_{i=1}^{n}$ be the parallel transport of some orthonormal frame along $\gamma(t)$. Then, for any $w \in \mathbb{R}^{n}$, we have that

$$
d \exp _{x}\left(v_{x}\right)\left(\sum w_{i} X_{i}(0)\right)=\sum_{i} \psi_{w}(\ell)_{i} X_{i}(\ell)
$$

where $\psi_{w}$ satisfies the following Jacobi equation along $\gamma(t)$ :

$$
\begin{aligned}
\frac{d^{2}}{d t^{2}} \psi_{w}(t)+R(t) \psi_{w}(t) & =0 \text { for all } 0 \leq t \leq \ell \\
\frac{d}{d t} \psi_{w}(0) & =\frac{w}{\ell} \\
\psi_{w}(0) & =0
\end{aligned}
$$

and $R(t)$ is defined in Definition 8.

Proof. We want to compute $d \exp _{x}\left(v_{x}\right)(w)$ for some $w \in T_{y} M$. By definition, we have that

$$
d \exp _{x}\left(v_{x}\right)(w)=\left.\frac{d}{d s} \gamma(t, s)\right|_{t=\ell, s=0}
$$

where $\gamma(t, s)=\exp _{x}\left(t v_{x} / \ell+s w\right)$. It is known that $\eta_{w}(t)=\left.\frac{d}{d s} \gamma(t, s)\right|_{s=0}$ is a Jacobi field given by the formula (Sec 2.1.2)

$$
\begin{aligned}
D_{t} D_{t} \eta_{w}+R\left(\eta_{w}, \gamma^{\prime}(t)\right) \gamma^{\prime}(t) & =0, \text { for } 0 \leq t \leq \ell \\
D_{t} \eta_{w}(0) & =w / \ell \\
\eta_{w}(0) & =0 .
\end{aligned}
$$

Recall that the parallel transport of an orthonormal frame remains orthonormal because

$$
\frac{d}{d t}\left\langle X_{i}(t), X_{j}(t)\right\rangle_{\gamma(t)}=\left\langle D_{t} X_{i}(t), X_{j}(t)\right\rangle_{\gamma(t)}+\left\langle X_{i}(t), D_{t} X_{j}(t)\right\rangle_{\gamma(t)}=0 .
$$


Since $X_{i}(t)$ is an orthonormal basis at $T_{\gamma(t)} M$, we can write

$$
\eta_{w}(t)=\sum_{i} \psi_{w, i}(t) X_{i}(t)
$$

Now, we need to verify $\psi_{w}$ satisfies (3.4). The second and last equation is immediate.

Since $\eta_{w}$ satisfies the ODE above, we have

$$
D_{t} D_{t} \sum_{i} \psi_{w, i}(t) X_{i}(t)+R\left(\sum_{i} \psi_{w, i}(t) X_{i}(t), \gamma^{\prime}(t)\right) \gamma^{\prime}(t)=0 .
$$

Since $X_{i}(t)$ is a parallel transport, we have that $D_{t} D_{t}\left(\psi_{w, i}(t) X_{i}(t)\right)=\frac{d^{2}}{d t^{2}} \psi_{w, i}(t) X_{i}(t)$ and hence

$$
\sum_{i} \frac{d^{2}}{d t^{2}} \psi_{w, i}(t) X_{i}(t)+\sum_{i} \psi_{w, i}(t) R\left(X_{i}(t), \gamma^{\prime}(t)\right) \gamma^{\prime}(t)=0
$$

Let $R_{i j}(t)=\left\langle R\left(X_{i}(t), \gamma^{\prime}(t)\right) \gamma^{\prime}(t), X_{j}(t)\right\rangle$. Since $R(t)$ is a symmetric matrix (Fact 5), we have

$$
\frac{d^{2}}{d t^{2}} \psi_{w}(t)+R(t) \psi_{w}(t)=0
$$

This verifies the first equation in (3.4).

Next, we have an elementary lemma about the determinant.

Lemma 35. Suppose that $E$ is a matrix (not necessarily symmetric) with $\|E\|_{2} \leq \frac{1}{4}$, we have

$$
|\log \operatorname{det}(I+E)-\operatorname{Tr} E| \leq\|E\|_{F}^{2} .
$$

Proof. Let $f(t)=\log \operatorname{det}(I+t E)$. Then, by Jacobi's formula, we have

$$
\begin{aligned}
f^{\prime}(t) & =\operatorname{Tr}\left((I+t E)^{-1} E\right), \\
f^{\prime \prime}(t) & =-\operatorname{Tr}\left((I+t E)^{-1} E(I+t E)^{-1} E\right) .
\end{aligned}
$$

Since $\|E\|_{2} \leq \frac{1}{4}$, we have that $\left\|(I+t E)^{-1}\right\|_{2} \leq \frac{4}{3}$ and hence

$$
\begin{aligned}
\left|f^{\prime \prime}(t)\right| & =\left|\operatorname{Tr}\left((I+t E)^{-1} E(I+t E)^{-1} E\right)\right| \\
& \leq\left|\operatorname{Tr}\left(E^{T}\left((I+t E)^{-1}\right)^{T}(I+t E)^{-1} E\right)\right| \\
& \leq 2\left|\operatorname{Tr}\left(E^{T} E\right)\right|=2\|E\|_{F}^{2} .
\end{aligned}
$$

The result follows from

$$
\begin{aligned}
f(1) & =f(0)+f^{\prime}(0)+\int_{0}^{1}(1-s) f^{\prime \prime}(s) d s \\
& =\operatorname{Tr}(E)+\int_{0}^{1}(1-s) f^{\prime \prime}(s) d s
\end{aligned}
$$

Using lemma 35 along with bounds on the solution to the Jacobi field equation (Lemma 42 in Sec. 3.8), we have the following: 
Lemma 36. Given a geodesic walk $\gamma(t)=\exp _{x}\left(\frac{t}{\ell} v_{x}\right)$ with step size $h$ satisfying $0<h \leq \frac{1}{n R}$ where $R=\max _{0 \leq t \leq \ell}\|R(t)\|_{F}$. We have that $d \exp _{x}\left(v_{x}\right)$ is invertible,

$$
\left|\log \operatorname{det}\left(\exp _{x}\left(v_{x}\right)\right)-\int_{0}^{\ell} \frac{s(\ell-s)}{\ell} \operatorname{Ric}\left(\gamma^{\prime}(s)\right) d s\right| \leq \frac{(n h R)^{2}}{6}
$$

and

$$
\left|\log \operatorname{det}\left(d \exp _{x}\left(v_{x}\right)\right)-\log \operatorname{det}\left(d \exp _{y}\left(v_{y}\right)\right)\right| \leq \frac{(n h R)^{2}}{3}
$$

If we further assume $V(\gamma) \leq V_{0}$ and $h \leq H$, then we have that $R \leq R_{1}$.

Proof. Let $\Psi$ be the solution of the ODE $\Psi^{\prime \prime}(t)+R(t) \Psi(t)=0, \Psi^{\prime}(0)=I / \ell$ and $\Psi(0)=0$. We know that $\|R(t)\|_{2} \leq\|R(t)\|_{F} \leq R$ for all $0 \leq t \leq \ell$. Hence, Lemma 42 shows that

$$
\begin{aligned}
\|\Psi(t)-I\|_{F} & \leq \max _{0 \leq s \leq \ell}\|R(s)\|_{F}\left(\frac{\ell^{3}}{5}\|I / \ell\|_{2}\right) \\
& \leq \frac{1}{5} n h R \leq \frac{1}{5}
\end{aligned}
$$

By the Lemma 35, we have that

$$
|\log \operatorname{det}(\Psi(\ell))-\operatorname{Tr}(\Psi(\ell)-I)| \leq\left(\frac{1}{5} n h R\right)^{2} .
$$

Now, we need to estimate $\operatorname{Tr}(\Psi(\ell)-I)$. Note that

$$
\begin{aligned}
\Psi(\ell) & =\Psi(0)+\Psi^{\prime}(0) \ell+\int_{0}^{\ell}(\ell-s) R(s) \Psi(s) d s \\
& =I+\int_{0}^{\ell}(\ell-s) R(s) \Psi(s) d s .
\end{aligned}
$$

Hence, we have

$$
\Psi(\ell)-I-\int_{0}^{\ell} \frac{s(\ell-s)}{\ell} R(s) d s=\int_{0}^{\ell}(\ell-s) R(s)\left(\Psi(s)-\frac{s}{\ell} I\right) d s .
$$

Using Lemma (3.6), we have

$$
\begin{aligned}
\left|\operatorname{Tr}\left(\Psi(\ell)-I-\int_{0}^{\ell} \frac{s(\ell-s)}{\ell} R(s) d s\right)\right| & \leq \int_{0}^{\ell}(\ell-s)\left|\operatorname{Tr} R(s)\left(\Psi(s)-\frac{s}{\ell} I\right)\right| d s \\
& \leq \int_{0}^{\ell}(\ell-s)\|R(s)\|_{F}\left\|\Psi(s)-\frac{s}{\ell} I\right\|_{F} d s \\
& \leq \frac{\ell^{2}}{2} \cdot R \cdot \frac{1}{5} R n h \leq \frac{(n h R)^{2}}{10} .
\end{aligned}
$$

Combining (3.7) and (3.8), we have that

$$
\left|\log \operatorname{det}(\Psi(\ell))-\int_{0}^{\ell} \frac{s(\ell-s)}{\ell} R(s) d s\right| \leq\left(\frac{1}{5} R n h\right)^{2}+\frac{(n h R)^{2}}{10} \leq \frac{(n h R)^{2}}{6} .
$$

By Lemma 34, for any $w$, we have that $d \exp _{x}\left(v_{x}\right)\left(\sum w_{i} X_{i}(0)\right)=\sum_{i} \psi_{w}(\ell)_{i} X_{i}(\ell)=\sum_{i}(\Psi(\ell) w)_{i} X_{i}(\ell)$. Since $\left\{X_{i}(t)\right\}_{i=1}^{n}$ are orthonormal, this shows that

$$
d \exp _{x}\left(v_{x}\right)=X(\ell) \Psi(\ell) X(0)^{T}
$$


where $X$ is the matrix $\left[X_{1}, X_{2}, \cdots, X_{n}\right]$. Since $\|\Psi(\ell)-I\|_{2} \leq \frac{1}{5}(3.6)$, we have that $\Psi(\ell)$ is invertible and so is $d \exp _{x}\left(v_{x}\right)$.

Since $X(\ell)$ and $X(0)$ are orthonormal, we have that

$$
\log \operatorname{det}\left(d \exp _{x}\left(v_{x}\right)\right)=\log \operatorname{det} \Psi(\ell)
$$

Therefore, this gives

$$
\left|\log \operatorname{det}\left(d \exp _{x}\left(v_{x}\right)\right)-\int_{0}^{\ell} \frac{s(\ell-s)}{\ell} \operatorname{Tr} R(s) d s\right| \leq \frac{(n h R)^{2}}{6} .
$$

By the definition of Ricci curvature and Fact 5, we have that

$$
\begin{aligned}
\operatorname{Tr} R(s) & =\sum_{i}\left\langle R\left(X_{i}(s), \gamma^{\prime}(s)\right) \gamma^{\prime}(s), X_{j}(s)\right\rangle \\
& =\operatorname{Ric}\left(\gamma^{\prime}(s)\right) .
\end{aligned}
$$

This gives the result (3.5).

Since the geodesic $\exp _{x}\left(t v_{x}\right)$ is the same as the geodesic $\exp _{y}\left(t v_{y}\right)$ except for swapping the parameterization, and since $\int_{0}^{\ell} \frac{s(\ell-s)}{\ell} \operatorname{Tr} R(s) d s$ is invariant under this swap, (3.5) implies that $\log \operatorname{det}\left(d \exp _{x}\left(v_{x}\right)\right)$ is close to $\log \operatorname{det}\left(d \exp _{y}\left(v_{y}\right)\right)$.

\subsection{Smoothness of one-step distributions}

Here we prove Theorem 25. Recall that the probability density of going from $x$ to $y$ is given by the following formula:

$$
p_{x}(y)=\sum_{v_{x}: \exp _{x}\left(v_{x}\right)=y} \operatorname{det}\left(d \exp _{x}\left(v_{x}\right)\right)^{-1} \sqrt{\frac{\operatorname{det}(g(y))}{(2 \pi h)^{n}}} \exp \left(-\frac{1}{2}\left\|\frac{v_{x}-\frac{h}{2} \mu(x)}{\sqrt{h}}\right\|_{x}^{2}\right)
$$

To simplify the calculation, we apply Lemma 36 and consider the following estimate of $p_{x}(y)$ instead

$$
\tilde{p}_{x}(y)=\sum_{v_{x}: \exp _{x}\left(v_{x}\right)=y} \sqrt{\frac{\operatorname{det}(g(y))}{(2 \pi h)^{n}}} \exp \left(-\int_{0}^{\ell} \frac{t(\ell-t)}{\ell} \operatorname{Ric}\left(\gamma_{v_{x}}^{\prime}(t)\right) d t-\frac{1}{2}\left\|\frac{v_{x}-\frac{h}{2} \mu(x)}{\sqrt{h}}\right\|_{x}^{2}\right)
$$

where $\gamma_{v_{x}}(t)$ be the geodesic from $\gamma_{v_{x}}(0)=x$ with $\gamma_{v_{x}}^{\prime}(0)=v_{x}$. Lemma 36 shows that $\left|\log \left(\tilde{p}_{x}(y) / p_{x}(y)\right)\right|$ is small and hence it suffices to prove the smoothness of $\tilde{p}_{x}(y)$.

Let $c(s)$ be an unit speed geodesic going from $x$ to some point $z$ very close to $x$. Lemma 44 shows that there is an unique vector field $v(s)$ on $c(s)$ such that $\exp _{c(s)}(v(s))=y$ and $v(0)=v_{x}$. Now, we define

$$
\zeta(v, s)=\sqrt{\frac{\operatorname{det}(g(y))}{(2 \pi h)^{n}}} \exp \left(-\int_{0}^{\ell} \frac{t(\ell-t)}{\ell} \operatorname{Ric}\left(\gamma_{s}^{\prime}(t)\right) d t-\frac{1}{2}\left\|\frac{v(s)-\frac{h}{2} \mu(c(s))}{\sqrt{h}}\right\|_{c(s)}^{2}\right)
$$

where $\gamma_{s}(t)=\exp _{c(s)}\left(\frac{t}{\ell} v(s)\right)$. Then, we have that

$$
\tilde{p}_{c(s)}(y)=\sum_{v: \exp _{c(s)}(v(s))=y} \zeta(v, s)
$$


and hence

$$
\frac{d}{d s} \tilde{p}_{c(s)}(y)=\sum_{v: \exp _{c(s)}(v(s))=y}\left(-\int_{0}^{\ell} \frac{t(\ell-t)}{\ell} \frac{d}{d s} \operatorname{Ric}\left(\gamma_{s}^{\prime}(t)\right) d t-\frac{1}{2} \frac{d}{d s}\left\|\frac{v(s)-\frac{h}{2} \mu(c(s))}{\sqrt{h}}\right\|_{c(s)}^{2}\right) \zeta(v, s) .
$$

Hence, it suffices to bound the terms in parenthesis.

In Lemma 38 , we analyze $\frac{d}{d s} \operatorname{Ric}\left(\gamma_{s}^{\prime}(t)\right)$ and prove that

$$
\left|\int_{0}^{\ell} \frac{t(\ell-t)}{\ell} \frac{d}{d s} \operatorname{Ric}\left(\gamma_{s}^{\prime}(t)\right) d t\right|=O\left(n h R_{2}\right)
$$

In Lemma 39, we analyze $\frac{d}{d s}\left\|\frac{v(s)-\frac{h}{2} \mu(c(s))}{\sqrt{h}}\right\|_{c(s)}^{2}$ and prove that

$$
\mathbb{E}_{V(\gamma) \leq V_{0}}\left|\frac{d}{d s}\left\|\frac{v(s)-\frac{h}{2} \mu(c(s))}{\sqrt{h}}\right\|_{c(s)}^{2}\right|_{s=0} \mid=O\left(D_{2} \sqrt{h}+\frac{1}{\sqrt{h}}+n h D_{0} R_{1}\right)
$$

where $\gamma$ is a random geodesic walk starting from $x$ (before the filtering step). This implies Thm. 29 , restated below for convenience.

Theorem 29. For $h \leq \min \left(H, \frac{1}{10^{6} n R_{1}}\right)$, then the one-step distributions $P_{x}, P_{z}$ from $x, z$ satisfy

$$
d_{T V}\left(P_{x}, P_{z}\right)=O\left(n h R_{2}+D_{2} \sqrt{h}+\frac{1}{\sqrt{h}}+n h D_{0} R_{1}\right) d(x, z)+\frac{1}{20} .
$$

Proof. Given $z$ such that $d(x, z)<\frac{V_{0}}{4 V_{1}}$. Let $c(s)$ be an unit speed minimal length geodesic connecting $x$ and $z, \tilde{p}_{c(s)}$ is defined by (3.9).

By (3.1), with probability $1-\frac{V_{0}}{100 V_{1}}$ in $y$, we have that $V(\gamma) \leq \frac{1}{2} V_{0}$. Let $Y$ be the set of $y$ such that $V(\gamma) \leq \frac{1}{2} V_{0}$. Since the distance from $x$ to $z$ is less than $\frac{V_{0}}{4 V_{1}}$ and $V(\gamma) \leq \frac{1}{2} V_{0}$ for those $y$, Lemma 36 shows there is a family of geodesic $\gamma_{s}$ which connects $c(s)$ to $y$. Furthermore, we have that $V\left(\gamma_{s}\right) \leq V_{0}$.

For any $y \in Y$, we have that $V\left(\gamma_{s}\right) \leq V_{0}$. By Lemma 36, we have that

$$
\exp \left(\frac{-1}{6}\left(n h R_{1}\right)^{2}\right) p_{c(s)}(y) \leq \tilde{p}_{c(s)}(y) \leq \exp \left(\frac{1}{6}\left(n h R_{1}\right)^{2}\right) p_{c(s)}(y) \text {. }
$$

Using our assumption on $h$, we have that

$$
\exp \left(3\left(n h R_{1}\right)^{2}\right) \leq C \stackrel{\text { def }}{=} 1.01 .
$$

Therefore, we have that

$$
\begin{aligned}
p_{x}(y)-p_{z}(y) & \leq\left(1-C^{-2}\right) p_{x}(y)+C^{-2} p_{x}(y)-C^{-1} \tilde{p}_{z}(y) \\
& \leq\left(1-C^{-2}\right) p_{x}(y)+C^{-1}\left(\tilde{p}_{x}(y)-\tilde{p}_{z}(y)\right) .
\end{aligned}
$$

Similarly, we have

$$
p_{x}(y)-p_{z}(y) \geq\left(1-C^{2}\right) p_{x}(y)+C\left(\tilde{p}_{x}(y)-\tilde{p}_{z}(y)\right) .
$$


Since $\int_{Y} p_{x}(y) d y \geq 1-\frac{V_{0}}{100 V_{1}}$, we have that

$$
\begin{aligned}
d_{T V}\left(p_{x}, p_{z}\right) & \leq \frac{V_{0}}{100 V_{1}}+\int_{Y}\left|p_{x}(y)-p_{z}(y)\right| d y \\
& \leq \frac{V_{0}}{100 V_{1}}+\frac{1}{50} \int_{Y}\left|p_{x}(y)\right| d y+2 \int\left|\tilde{p}_{x}(y)-\tilde{p}_{z}(y)\right| d y \\
& \leq \frac{V_{0}}{20 V_{1}}+2 \iint_{Y}\left|\frac{d}{d s} \tilde{p}_{c(s)}(y)\right| d y d s .
\end{aligned}
$$

Recall from (3.10) that

$$
\frac{d}{d s} \tilde{p}_{c(s)}(y)=\sum_{v: \exp _{c(s)}(v(s))=y}\left(-\int_{0}^{\ell} \frac{t(\ell-t)}{\ell} \frac{d}{d s} \operatorname{Ric}\left(\gamma_{s}^{\prime}(t)\right) d t-\frac{1}{2} \frac{d}{d s}\left\|\frac{v(s)-\frac{h}{2} \mu(c(s))}{\sqrt{h}}\right\|_{c(s)}^{2}\right) \zeta(v, s) .
$$

Using Lemma 36 again, we have that $\zeta(v, s) \leq C \cdot p(c(s) \stackrel{v(s)}{\rightarrow} y) \leq 2 p(c(s) \stackrel{v(s)}{\rightarrow} y)$ and hence

$$
\left|\frac{d}{d s} \tilde{p}_{c(s)}(y)\right| \leq 2 \sum_{v: \exp _{c(s)}(v(s))=y}\left(\left|\int_{0}^{\ell} \frac{t(\ell-t)}{\ell} \frac{d}{d s} \operatorname{Ric}\left(\gamma_{s}^{\prime}(t)\right) d t\right|+\left|\frac{d}{d s}\left\|\frac{v(s)-\frac{h}{2} \mu(c(s))}{\sqrt{h}}\right\|_{c(s)}^{2}\right|\right) p(c(s) \stackrel{v(s)}{\rightarrow} y) .
$$

Since $V\left(\gamma_{s}\right) \leq V_{0}$, we can use Lemma 38 to get

$$
\left|\int_{0}^{\ell} \frac{t(\ell-t)}{\ell} \frac{d}{d s} \operatorname{Ric}\left(\gamma_{s}^{\prime}(t)\right) d t\right| \leq O\left(n h R_{2}\right)
$$

Hence, we have that

$$
\begin{aligned}
& \int_{Y}\left|\frac{d}{d s} \tilde{p}_{c(s)}(y)\right| d y \\
\leq & n h R_{2} \int_{Y} \sum_{v: \exp _{c(s)}(v(s))=y} p(c(s) \stackrel{v(s)}{\rightarrow} y) d y+2 \int_{Y} \sum_{v: \exp _{c(s)}(v(s))=y}\left|\frac{d}{d s} \| \frac{v(s)-\frac{h}{2} \mu(c(s))}{\sqrt{h}}\right|_{c(s)}^{2} \mid p(c(s) \stackrel{v(s)}{\rightarrow} y) d y .
\end{aligned}
$$

For the first term, we note that $\int_{Y} \sum_{v: \exp _{c(s)}(v(s))=y} p(c(s) \stackrel{v(s)}{\rightarrow} y) d y \leq 1$.

For the second term, we note that $y \in Y$ implies $V\left(\gamma_{s}\right) \leq V_{0}$. Hence, we have that

$$
\begin{aligned}
& \int_{Y} \sum_{v: \exp _{c(s)}(v(s))=y}\left|\frac{d}{d s} \| \frac{v(s)-\frac{h}{2} \mu(c(s))}{\sqrt{h}}\right|_{c(s)}^{2} \mid p(c(s) \stackrel{v(s)}{\rightarrow} y) d y \\
\leq & \int_{V\left(\gamma_{s}\right) \leq V_{0}} \sum_{v: \exp _{c(s)}(v(s))=y}\left|\frac{d}{d s} \| \frac{v(s)-\frac{h}{2} \mu(c(s))}{\sqrt{h}}\right|_{c(s)}^{2} \mid p(c(s) \stackrel{v(s)}{\rightarrow} y) d y \\
= & \left.\mathbb{E}_{V\left(\gamma_{s}\right) \leq V_{0}}\left|\frac{d}{d s} \| \frac{v(s)-\frac{h}{2} \mu(c(s))}{\sqrt{h}}\right|\right|_{c(s)} ^{2} \mid \\
= & O\left(D_{2} \sqrt{h}+\frac{1}{\sqrt{h}}+n h D_{0} R_{1}\right)
\end{aligned}
$$


where we used Lemma 39 at the end.

Hence, we have that

$$
\int_{Y}\left|\frac{d}{d s} \tilde{p}_{c(s)}(y)\right| d y=O\left(n h R_{2}+D_{2} \sqrt{h}+\frac{1}{\sqrt{h}}+n h D_{0} R_{1}\right) .
$$

Putting this into (3.11), we get

$$
d_{T V}\left(p_{x}, p_{z}\right)=O\left(n h R_{2}+D_{2} \sqrt{h}+\frac{1}{\sqrt{h}}+n h D_{0} R_{1}\right) d(x, z)+\frac{V_{0}}{20 V_{1}}
$$

for any $d(x, z)<\frac{V_{0}}{4 V_{1}}$. By summing along a path, for any $x$ and $z$ and using that $V_{1} \geq V_{0}$, we have that

$$
d_{T V}\left(p_{x}, p_{z}\right)=O\left(n h R_{2}+D_{2} \sqrt{h}+\frac{1}{\sqrt{h}}+n h D_{0} R_{1}+1\right) d(x, z)+\frac{1}{20} .
$$

Definition 37. Given a manifold $M$ and a family of geodesic $\gamma_{s}(t)$ with step size $h$ such that $h \leq H$ and $V\left(\gamma_{0}\right) \leq V_{0}$. Let $R_{2}$ be a constant depending on the manifold $M$ and the step size $h$ such that for any $t$ such that $0 \leq t \leq \ell$, any curve $c(s)$ starting from $\gamma(t)$ and any vector field $v(s)$ on $c(s)$ with $v(0)=\gamma^{\prime}(t)$, we have that

$$
\left|\frac{d}{d s} \operatorname{Ric}(v(s))\right|_{s=0} \mid \leq\left(\left.\left.|| \frac{d c}{d s}\right|_{s=0}\|+\ell\| D_{s} v\right|_{s=0} \|\right) R_{2} .
$$

Lemma 38. For $h \leq \min \left(H, \frac{1}{2 n R_{1}}\right)$ and $V\left(\gamma_{s}\right) \leq V_{0}$, we have

$$
\left|\int_{0}^{\ell} \frac{t(\ell-t)}{\ell} \frac{d}{d s} \operatorname{Ric}\left(\gamma_{s}^{\prime}(t)\right) d t\right| \leq O\left(n h R_{2}\right)
$$

where $\gamma_{s}$ is as defined in the beginning of Section 3.7.

Proof. By Definition 37, we have that

$$
\left|\frac{d}{d s} \operatorname{Ric}\left(\gamma_{s}^{\prime}(s)\right)\right| \leq\left(\left\|\frac{d}{d s} \gamma_{s}(0)\right\|+\ell\left\|D_{s} \gamma_{s}^{\prime}(0)\right\|\right) R_{2} .
$$

By definition of $\gamma_{s}$, we have that $\frac{d}{d s} \gamma_{s}(0)=\frac{d}{d s} \exp _{c(s)}(0)=\frac{d}{d s} c(s)$ is an unit vector and hence $\left\|\frac{d}{d s} \gamma_{s}(0)\right\|=1$. To bound the second term, we note that $\psi(t)=\frac{\partial}{\partial s} \gamma_{s}$ is a Jacobi field and Lemma 41 shows that

$$
\left\|\psi(t)-\psi(0)-t D_{t} \psi(0)\right\| \leq \lambda t^{2}\|\psi(0)\|+\frac{\lambda t^{3}}{5}\left\|D_{t} \psi(0)\right\|
$$

where $\lambda=\max _{0 \leq s \leq \ell}\|R(s)\|_{F} \leq R_{1}$. Putting $t=\ell, \psi(\ell)=0$ and $\lambda \ell^{2} \leq R_{1} n h \leq \frac{1}{2}$, we have

$$
\left\|\psi(0)+\ell D_{t} \psi(0)\right\| \leq \frac{1}{2}\|\psi(0)\|+\frac{\ell}{10}\left\|D_{t} \psi(0)\right\| .
$$

Hence, we have that

$$
\left\|D_{t} \psi(0)\right\| \leq \frac{3}{\ell}\|\psi(0)\|=\frac{3}{\ell} .
$$

Using these, we have that $\|\psi(t)\|=O(1)$ and $\left\|D_{t} \psi(t)\right\|=O\left(\frac{1}{\ell}\right)$ for $0 \leq t \leq \ell$. 
Fix any $0 \leq t \leq \ell$, we define $c(s)=\gamma_{s}(t)$ and $v(s)=\gamma_{s}^{\prime}(t)$. Using $\psi(t)=\frac{d c}{d s}$ and $D_{t} \psi(t)=$ $D_{t} \frac{d}{d s} \gamma_{s}=D_{s} v,(3.12)$ shows that

$$
\left|\frac{d}{d s} \operatorname{Ric}\left(\gamma_{s}^{\prime}(s)\right)\right| \leq O\left(R_{2}\right)
$$

Lemma 39. Given an unit geodesic $c(s)$ from $x$ to $z$. For any geodesic $\gamma(t)$ from $x$, we define a corresponding vector field $v(s)$ on $c(s)$ such that $\gamma(t)=\exp _{x}\left(\frac{t}{\ell} v(0)\right)$ and $\exp _{c(s)}(v(s))=\gamma(\ell)$ for all $s$ near 0 . Suppose that $h \leq H$, then we have that

$$
\mathbb{E}_{V(\gamma) \leq V_{0}}\left|\frac{d}{d s}\left\|\frac{v(s)-\frac{h}{2} \mu(c(s))}{\sqrt{h}}\right\|_{c(s)}^{2}\right|_{s=0} \mid=O\left(D_{2} \sqrt{h}+\frac{1}{\sqrt{h}}+n h D_{0} R_{1}\right)
$$

where $\gamma$ is a random geodesic walk starting from $x$ (before the filtering step).

Proof. Note that

$$
\left.\frac{d}{d s}\left\|\frac{v(s)-\frac{h}{2} \mu(c(s))}{\sqrt{h}}\right\|_{c(s)}^{2}\right|_{s=0}=\frac{2}{h}\left\langle\left. D_{s} v\right|_{s=0}-\left.\frac{h}{2} D_{s} \mu\right|_{s=0}, v-\frac{h}{2} \mu\right\rangle .
$$

Since $v-\frac{h}{2} \mu$ is a random Gaussian vector $N(0, n h I)$ in $T_{x} M$ independent of $D_{s} \mu$, we have that

$$
\mathbb{E}_{\gamma}\left|\left\langle\left.\frac{h}{2} D_{s} \mu\right|_{s=0}, v-\frac{h}{2} \mu\right\rangle\right| \leq O\left(\frac{\left\|\left.\frac{h}{2} D_{s} \mu\right|_{s=0}\right\| \sqrt{n h}}{\sqrt{n}}\right) \leq O\left(h^{3 / 2} D_{2}\right) .
$$

By (3.1), we have that $\mathbb{P}\left(V(\gamma)<V_{0}\right) \geq \frac{1}{2}$ and hence

$$
\mathbb{E}_{V(\gamma) \leq V_{0}}\left|\left\langle\left.\frac{h}{2} D_{s} \mu\right|_{s=0}, v-\frac{h}{2} \mu\right\rangle\right|=O\left(h^{3 / 2} D_{2}\right) .
$$

By Lemma 43, we know that $\left.D_{s} v\right|_{s=0}=-c^{\prime}+\zeta$ where $\zeta \perp v(0)$ and $\|\zeta\| \leq \frac{3}{2} n h R_{1}$ when $V(\gamma) \leq V_{0}$. Since $v-\frac{h}{2} \mu$ is a random Gaussian vector independent of $c^{\prime}$, we have that

$$
\begin{aligned}
\mathbb{E}_{V(\gamma) \leq V_{0}}\left|\left\langle D_{s} v, v-\frac{h}{2} \mu\right\rangle\right| & \leq \mathbb{E}_{V(\gamma) \leq V_{0}}\left|\left\langle c^{\prime}, v-\frac{h}{2} \mu\right\rangle\right|+\frac{h}{2} \mathbb{E}|\langle\zeta, \mu\rangle| \\
& \leq O\left(\frac{\left\|c^{\prime}\right\| \sqrt{n h}}{\sqrt{n}}\right)+n h^{2} R_{1}\|\mu\| \\
& \leq O\left(\sqrt{h}+n h^{2} D_{0} R_{1}\right) .
\end{aligned}
$$

Combining the bounds (3.13), (3.14) and (3.15), we have that

$$
\left.\mathbb{E}_{V(\gamma) \leq V_{0}}\left|\frac{d}{d s} \| \frac{v(s)-\frac{h}{2} \mu(c(s))}{\sqrt{h}}\right|_{c(s)}^{2}\right|_{s=0} \mid \leq O\left(D_{2} \sqrt{h}+\frac{1}{\sqrt{h}}+n h D_{0} R_{1}\right) .
$$




\subsection{Approximate solution of Jacobi field equations}

Let $\gamma(t)$ be a geodesic and $\left\{X_{i}(t)\right\}_{i=1}^{n}$ be the parallel transport of some orthonormal frame along $\gamma(t)$. As we demonstrated in the proof of Lemma 34, Jacobi fields can be expressed as linear combinations of $X_{i}$ and the coefficients are given by the following matrix ODE:

$$
\begin{aligned}
\frac{d^{2}}{d t^{2}} \psi(t)+R(t) \psi(t) & =0, \\
\frac{d}{d t} \psi(0) & =\beta, \\
\psi(0) & =\alpha
\end{aligned}
$$

where $\psi(t), \alpha, \beta \in \mathbb{R}^{n}$ and $R(t)$ is defined in Definition 8 .

In this section, we give estimates for Jacobi equations (3.16). The estimates we get can be viewed as small variants of the Rauch comparison theorem (See [13, Sec 4.5]). The Rauch comparison theorem gives upper and lower bound on the magnitude of Jacobi field. Our bounds instead show how fast Jacobi field deviates from its linear approximation.

First, we give a basic bound on the solution in terms of hyperbolic sine and cosine functions, which is a direct consequence of the Rauch comparison theorem. We include a direct proof of this for completeness.

Lemma 40. Let $\psi$ be the solution of (3.16). Suppose that $\|R(t)\|_{2} \leq \lambda$ for all $0 \leq t \leq \ell$. Then, we have that

$$
\|\psi(t)\|_{2} \leq\|\alpha\|_{2} \cosh (\sqrt{\lambda} t)+\frac{\|\beta\|_{2}}{\sqrt{\lambda}} \sinh (\sqrt{\lambda} t)
$$

for all $0 \leq t \leq \ell$.

Proof. Note that

$$
\begin{aligned}
\psi(t) & =\psi(0)+\psi^{\prime}(0) t+\int_{0}^{t}(t-s) \psi^{\prime \prime}(s) d s \\
& =\alpha+\beta t-\int_{0}^{t}(t-s) R(s) \psi(s) d s .
\end{aligned}
$$

Let $a(t)=\|\psi(t)\|_{2}$, then we have that

$$
a(t) \leq\|\alpha\|_{2}+\|\beta\|_{2} t+\lambda \int_{0}^{t}(t-s) a(s) d s .
$$

Let $\bar{a}(t)$ be the solution of the integral equation

$$
\bar{a}(t)=\|\alpha\|_{2}+\|\beta\|_{2} t+\lambda \int_{0}^{t}(t-s) \bar{a}(s) d s .
$$

By induction, we have that $a(t) \leq \bar{a}(t)$ for all $t \geq 0$. By taking derivatives on both sides, we have that

$$
\bar{a}^{\prime \prime}(t)=\lambda \bar{a}(t), \bar{a}(0)=\|\alpha\|_{2}, \bar{a}^{\prime}(0)=\|\beta\|_{2} .
$$

Solving these equations, we have

$$
\|\psi(t)\|_{2}=a(t) \leq \bar{a}(t)=\|\alpha\|_{2} \cosh (\sqrt{\lambda} t)+\frac{\|\beta\|_{2}}{\sqrt{\lambda}} \sinh (\sqrt{\lambda} t)
$$

for all $t \geq 0$. 
Next, we give an approximate solution of (3.16).

Lemma 41. Let $\psi$ be the solution of (3.16). Suppose that $\|R(t)\|_{2} \leq \lambda$ for all $0 \leq t \leq \frac{1}{\sqrt{\lambda}}$. For any $0 \leq t \leq \frac{1}{\sqrt{\lambda}}$, we have that

$$
\|\psi(t)-\alpha-\beta t\|_{2} \leq \lambda t^{2}\|\alpha\|_{2}+\frac{\lambda t^{3}}{5}\|\beta\|_{2}
$$

and

$$
\left\|\psi^{\prime}(t)-\beta\right\|_{2} \leq 2 \lambda t\|\alpha\|_{2}+\frac{3 \lambda t^{2}}{5}\|\beta\|_{2}
$$

Proof. Note that

$$
\psi(t)=\alpha+\beta t-\int_{0}^{t}(t-s) R(s) \psi(s) d s
$$

Using Lemma 40 and $\|R(t)\|_{2} \leq \lambda$, we have that

$$
\begin{aligned}
\|\psi(t)-\alpha-\beta t\|_{2} & \leq \lambda \int_{0}^{t}(t-s)\|\psi(s)\|_{2} d s \\
& \leq \lambda \int_{0}^{t}(t-s)\left(\|\alpha\|_{2} \cosh (\sqrt{\lambda} s)+\frac{\|\beta\|_{2}}{\sqrt{\lambda}} \sinh (\sqrt{\lambda} s)\right) d s \\
& =\|\alpha\|_{2}(\cosh (\sqrt{\lambda} t)-1)+\frac{\|\beta\|_{2}}{\sqrt{\lambda}}(\sinh (\sqrt{\lambda} t)-\sqrt{\lambda} t)
\end{aligned}
$$

Since $0 \leq t \leq \frac{1}{\sqrt{\lambda}}$, we have that $|\cosh (\sqrt{\lambda} t)-1| \leq \lambda t^{2}$ and $|\sinh (\sqrt{\lambda} t)-\sqrt{\lambda} t| \leq \frac{\lambda^{3 / 2} t^{3}}{5}$. This gives the result.

Similarly, we have that

$$
\psi^{\prime}(t)=\psi^{\prime}(0)+\int_{0}^{t} \psi^{\prime \prime}(s) d s=\psi^{\prime}(0)+\int_{0}^{t} R(s) \psi(s) d s .
$$

and hence

$$
\begin{aligned}
\left\|\psi^{\prime}(t)-\beta\right\|_{2} & \leq \lambda \int_{0}^{t}\left(\|\alpha\|_{2} \cosh (\sqrt{\lambda} s)+\frac{\|\beta\|_{2}}{\sqrt{\lambda}} \sinh (\sqrt{\lambda} s)\right) d s \\
& \leq \sqrt{\lambda}\|\alpha\|_{2} \sinh (\sqrt{\lambda} t)+\|\beta\|_{2}(\cosh (\sqrt{\lambda} t)-1) \\
& \leq 2 \lambda t\|\alpha\|_{2}+\frac{3}{5} \lambda t^{2}\|\beta\|_{2}
\end{aligned}
$$

The following is a matrix version of the above result.

Lemma 42. Let $\Psi$ be the solution of

$$
\begin{aligned}
\frac{d^{2}}{d t^{2}} \Psi(t)+R(t) \Psi(t) & =0 \\
\frac{d}{d t} \Psi(0) & =B \\
\Psi(0) & =A
\end{aligned}
$$


where $\Psi, A$ and $B$ are matrices with a compatible size. Suppose that $\|R(t)\|_{2} \leq \lambda$. For any $0 \leq t \leq$ $\frac{1}{\sqrt{\lambda}}$, we have that

$$
\|\Psi(t)-A-B t\|_{F} \leq \max _{0 \leq s \leq t}\|R(s)\|_{F}\left(t^{2}\|A\|_{2}+\frac{t^{3}}{5}\|B\|_{2}\right)
$$

Proof. Note that $\Psi(t) x$ is the solution of (3.16) with $\beta=B x$ and $\alpha=A x$. Therefore, Lemma 40 shows that

$$
\begin{aligned}
\|\Psi(t) x\|_{2} & \leq\|A x\|_{2} \cosh (\sqrt{\lambda} t)+\frac{\|B x\|_{2}}{\sqrt{\lambda}} \sinh (\sqrt{\lambda} t) \\
& \leq\left(\|A\|_{2} \cosh (\sqrt{\lambda} t)+\frac{\|B\|_{2}}{\sqrt{\lambda}} \sinh (\sqrt{\lambda} t)\right)\|x\|_{2}
\end{aligned}
$$

for all $x$. Therefore, we have that

$$
\Psi(t)^{T} \Psi(t) \preceq\left(\|A\|_{2} \cosh (\sqrt{\lambda} t)+\frac{\|B\|_{2}}{\sqrt{\lambda}} \sinh (\sqrt{\lambda} t)\right)^{2} I .
$$

Hence, we have that

$$
\Psi(t) \Psi(t)^{T} \preceq\left(\|A\|_{2} \cosh (\sqrt{\lambda} t)+\frac{\|B\|_{2}}{\sqrt{\lambda}} \sinh (\sqrt{\lambda} t)\right)^{2} I .
$$

Using this, we have that

$$
\begin{aligned}
\|\Psi(t)-A-B t\|_{F} & =\left\|\int_{0}^{t}(t-s) R(s) \Psi(s) d s\right\|_{F} \\
& \leq \int_{0}^{t}(t-s) \sqrt{\operatorname{Tr} \Psi^{T}(s) R^{T}(s) R(s) \Psi(s)} d s \\
& =\int_{0}^{t}(t-s) \sqrt{\operatorname{Tr} R(s) \Psi(s) \Psi^{T}(s) R^{T}(s)} d s \\
& \leq \int_{0}^{t}(t-s)\left(\|A\|_{2} \cosh (\sqrt{\lambda} s)+\frac{\|B\|_{2}}{\sqrt{\lambda}} \sinh (\sqrt{\lambda} s)\right)\|R(s)\|_{F} d s . \\
& \leq \max _{0 \leq s \leq t}\|R(s)\|_{F} \int_{0}^{t}(t-s)\left(\|A\|_{2} \cosh (\sqrt{\lambda} s)+\frac{\|B\|_{2}}{\sqrt{\lambda}} \sinh (\sqrt{\lambda} s)\right) d s \\
& =\max _{0 \leq s \leq t}\|R(s)\|_{F}\left(\|A\|_{2}(\cosh (\sqrt{\lambda} t)-1)+\frac{\|B\|_{2}}{\sqrt{\lambda}}(\sinh (\sqrt{\lambda} t)-\sqrt{\lambda} t)\right) .
\end{aligned}
$$

Since $0 \leq t \leq \frac{1}{\sqrt{\lambda}}$, we have that $|\cosh (\sqrt{\lambda} t)-1| \leq \lambda t^{2}$ and $|\sinh (\sqrt{\lambda} t)-\sqrt{\lambda} t| \leq \frac{\lambda^{3 / 2} t^{3}}{5}$. This gives the result.

\subsection{Almost one-to-one correspondence of geodesics}

We do not know if every pair $x, y \in M$ has a unique geodesic connecting $x$ and $y$. Due to this, the probability density $p_{x}$ at $y$ on $\mathcal{M}$ can be the sum over all possible geodesics connect $x$ and $y$. The goal of this section is to show there is a 1-1 map between geodesics paths connecting $x$ to $y$ as we move $x$. 
Lemma 43. Given a geodesic $\gamma(t)=\exp _{x}\left(\frac{t}{\ell} v_{x}\right)$. Let the end points $x=\gamma(0)$ and $y=\gamma(\ell)$. Suppose that $\ell^{2} \leq \frac{1}{R}$ where $R=\|R(t)\|_{F}$ and $R(t)$ defined in Definition 8, then there is an unique smooth invertible function $v: U \subset M \rightarrow V \subset T_{x} M$ such that

$$
y=\exp _{z}(v(z))
$$

for any $z \in U$ where $U$ is a neighborhood of $x$ and $V$ is a neighborhood of $v_{x}=v(x)$. Furthermore, for any $\eta=\alpha v_{x}+\eta_{2}$ with $\eta_{2} \perp v_{x}$ and scale $\alpha$, we have that

$$
\nabla_{\eta} v(x)=-\eta+\zeta
$$

where $\|\zeta\|_{x} \leq \frac{3}{2} \ell^{2} R\left\|\eta_{2}\right\|_{x} \leq \frac{3}{2}\left\|\eta_{2}\right\|_{x}$ and $\zeta \perp v_{x}$. In particular, we have that $\left\|\nabla_{\eta} v(x)\right\|_{x} \leq \frac{5}{2}\|\eta\|_{x}$. Proof. Consider the smooth function $f(z, w)=\exp _{z}(w)$. From Lemma 36, the differential of $w$ at $\left(x, v_{x}\right)$ on the $w$ variables, i.e. $d \exp _{x}\left(v_{x}\right)$, is invertible. Hence, the implicit function theorem shows that there is a open neighborhood $U$ of $x$ and a unique function $v$ on $U$ such that $f(z, v(z))=$ $f\left(x, v_{x}\right)$, i.e. $y=\exp _{x}\left(v_{x}\right)=\exp _{z}(v(z))$.

To compute $\nabla_{\eta} v$, let $c(s)$ be a geodesic starting from $x$ with $c^{\prime}(0)=\eta$ and $c(t, s)=\exp _{c(s)}\left(\frac{t}{\ell} v(c(s))\right)$ be a family of geodesics with the end points $c(\ell, s)=\exp _{c(s)}(v(c(s)))=y$. Note that $\psi(t) \stackrel{\text { def }}{=}$ $\left.\frac{\partial c(t, s)}{\partial s}\right|_{s=0}$ satisfies the Jacobi field equation

$$
D_{t}^{2} \psi(t)+R\left(\psi, \frac{\partial c}{\partial t}\right) \frac{\partial c}{\partial t}=0
$$

Let $\chi=\nabla_{\eta} v(x)$, we know that

$$
\begin{aligned}
\psi(0) & =\left.\frac{\partial c(0, s)}{\partial s}\right|_{s=0}=\gamma^{\prime}(0)=\eta \\
D_{t} \psi(0) & =\left.D_{t} \frac{\partial c(t, s)}{\partial s}\right|_{t, s=0}=\left.D_{s} \frac{\partial c(t, s)}{\partial t}\right|_{t, s=0}=\frac{1}{\ell} \nabla_{\eta} v(x)=\frac{\chi}{\ell}, \\
\psi(\ell) & =\left.\frac{\partial c(\ell, s)}{\partial s}\right|_{s=0}=0
\end{aligned}
$$

where we used $D_{t} \frac{\partial}{\partial s}=D_{s} \frac{\partial}{\partial t}$ (torsion free-ness, Fact 3).

From Fact 7 , we know that $\psi$ can be split into the tangential part $\psi_{1}$ and the normal part $\psi_{2}$. For the tangential part $\psi_{1}$, we know that

$$
\begin{aligned}
\psi_{1}(t) & =\left(\left\langle\psi(0), \gamma^{\prime}(0)\right\rangle_{\gamma(0)}+\left\langle D_{t} \psi(0), \gamma^{\prime}(0)\right\rangle_{\gamma(0)} t\right) \gamma^{\prime}(t) \\
& =\left(\left\langle\eta, v_{x}\right\rangle_{x}+\left\langle\frac{\chi}{\ell}, v_{x}\right\rangle_{x} t\right) \gamma^{\prime}(t) \\
& =\left(\alpha\left\|v_{x}\right\|_{x}^{2}+\left\langle\frac{\chi}{\ell}, v_{x}\right\rangle_{x} t\right) \gamma^{\prime}(t) .
\end{aligned}
$$

Since $\psi_{1}(\ell)=0$, we have that

$$
\left\langle\chi, v_{x}\right\rangle=-\alpha\left\|v_{x}\right\|_{x}^{2}
$$

For the normal part $\psi_{2}$, it is easier to calculate using orthogonal frames. Similar to Lemma 34, we pick an arbitrary orthogonal frame $X_{i}(t)$ parallel transported along the curve $\gamma(t)$ and let $\bar{\psi}(t)$ be $\psi_{2}(t)$ represented in that orthogonal frame. Hence, we have that

$$
\begin{aligned}
\frac{d^{2}}{d t^{2}} \bar{\psi}(t)+R(t) \bar{\psi}(t) & =0, \\
\bar{\psi}(0) & =\bar{\eta}_{2}, \\
\bar{\psi}^{\prime}(0) & =\frac{\bar{\chi}_{2}}{\ell}, \\
\bar{\psi}(\ell) & =0
\end{aligned}
$$


where $\bar{\chi}_{2}=\bar{\chi}-\left\langle\chi, \frac{v_{x}}{\left\|v_{x}\right\|}\right\rangle_{x} \frac{\bar{v}_{x}}{\left\|v_{x}\right\|}=\bar{\chi}+\alpha \bar{v}_{x}$ by (3.17) and $R(t)_{i j}=\left\langle R\left(X_{i}(t), \gamma^{\prime}(t)\right) \gamma^{\prime}(t), X_{j}(t)\right\rangle$. Since $\|R(t)\|_{2} \leq\|R(t)\|_{F} \leq R$ and $\ell^{2} \leq \frac{1}{R}$, Lemma 41 shows that

$$
\begin{aligned}
\left\|\bar{\eta}_{2}+\bar{\chi}_{2}\right\|_{2}=\left\|\bar{\psi}(\ell)-\bar{\eta}_{2}-\bar{\chi}_{2}\right\|_{2} & \leq R \ell^{2}\left\|\bar{\eta}_{2}\right\|_{2}+\frac{R \ell^{2}}{5}\left\|\bar{\chi}_{2}\right\|_{2} \\
& \leq \frac{6}{5} R \ell^{2}\left\|\bar{\eta}_{2}\right\|_{2}+\frac{R \ell^{2}}{5}\left\|\bar{\eta}_{2}+\bar{\chi}_{2}\right\|_{2} .
\end{aligned}
$$

Hence, we have that $\left\|\bar{\eta}_{2}+\bar{\chi}_{2}\right\|_{2} \leq \frac{3}{2} R \ell^{2}\left\|\bar{\eta}_{2}\right\|_{2}$. Therefore, we have that

$$
\begin{aligned}
\nabla_{\eta} v(x) & =\chi \\
& =-\alpha v_{x}+\chi_{2} \\
& =-\eta+\left(\eta_{2}+\chi_{2}\right)
\end{aligned}
$$

where $\left\|\chi_{2}+\eta_{2}\right\|_{x} \leq \frac{3}{2} R \ell^{2}\left\|\eta_{2}\right\|_{x}$. Furthermore, we have that both $\eta_{2}$ and $\chi_{2}$ are orthogonal to $\eta$.

Remark. If the above lemma holds without the assumption $\ell^{2} \leq \frac{1}{R}$, this would imply uniqueness of geodesics.

The following lemma shows there is a 1-1 map between geodesics paths connecting $x$ to $y$ as we move $x$. When we move $x$, the geodesic $\gamma$ from $x$ to $y$ changes and hence we need to bound $V(\gamma)$.

Lemma 44. Given a geodesic $\gamma(t)=\exp _{x}\left(\frac{t}{\ell} v_{x}\right)$ with step size $h$ satisfying $h \leq \min \left(H, \frac{1}{2 n R_{1}}\right)$, let $c(s)$ be any geodesic starting at $\gamma(0)$. Let $x=c(0)=\gamma(0)$ and $y=\gamma(1)$. Suppose that the length of $c(s)$ is less than $\frac{V_{0}}{4 V_{1}}$ and $V(\gamma) \leq \frac{V_{0}}{2}$. Then, there is a unique vector field $v$ on $c$ such that

$$
y=\exp _{c(s)}(v(s))
$$

Furthermore, this vector field is uniquely determined by the geodesic $c(s)$ and any $v(s)$ on this vector field. Also, we have that $V\left(\exp _{c(s)}(v(s))\right) \leq V_{0}$ for all $s$.

Proof. Let $s_{\max }$ be the supremum of $s$ such that $v(s)$ can be defined continuously such that $y=\exp _{c(s)}(v(s))$ and $V\left(\gamma_{s}\right) \leq V_{0}$ where $\gamma_{s}(t)=\exp _{c(s)}\left(\frac{t}{\ell} v(s)\right)$. Lemma 43 shows that there is a neighborhood $N$ at $x$ and a vector field $u$ on $N$ such that for any $z \in N$, we have that

$$
y=\exp _{z}(u(z))
$$

Also, this lemma shows that $u(s)$ is smooth and hence the parameter $V_{1}$ shows that $V\left(\gamma_{s}\right)$ is Lipschitz in $s$. Therefore, $V\left(\gamma_{s}\right) \leq V_{0}$ for a small neighborhood of 0 . Hence $s_{\max }>0$.

Now, we show $s_{\max }>1$ by contraction. By the definition of $s_{\max }$, we have that $V\left(\gamma_{s}\right) \leq V_{0}$ for any $0 \leq s<s_{\max }$. Hence, we can apply Lemma 43 with $R=R_{1}$. In particular, this shows that $\left\|D_{s} v(s)\right\|=\left\|\nabla_{\frac{d}{d s} c} u(x)\right\| \leq \frac{5}{2}\left\|\frac{d}{d s} c\right\|=\frac{5}{2} L$ where $L$ is the length of $c$. Therefore, the function $v$ is Lipschitz and hence $v\left(s_{\max }\right)$ is well-defined and $V\left(\gamma_{s_{\max }}\right) \leq V_{0}$ by continuity. Hence, we can apply Lemma 43 at $v\left(s_{\max }\right)$ and extend the domain of $v(s)$ beyond $s_{\max }$.

To bound $V\left(\gamma_{s}\right)$, we note that $\left\|D_{s} \gamma_{s}^{\prime}\right\|=\frac{1}{\ell}\left\|D_{s} v(s)\right\| \leq \frac{5}{2 \ell} L$ and $\left\|\frac{d}{d s} c\right\|=L$. Hence, $\left|\frac{d}{d s} V\left(\gamma_{s}\right)\right| \leq$ $\left(L+\frac{5}{2} L\right) V_{1}$ by the definition of $V_{1}$. Therefore, if $L \leq \frac{V_{0}}{4 V_{1}}$, we have that $V\left(\gamma_{s}\right) \leq V(\gamma)+\frac{V_{0}}{2} \leq V_{0}$ for all $s \leq 1.1$ whenever $v(s)$ is defined. Therefore, this draws a contradiction that $s_{\max }$ is not the supremum. Hence, $s_{\max } \geq 1$.

The uniqueness follows from Lemma 43. 


\subsection{Implementation}

Here, we explain in high level how to implement the geodesic walk in general via an efficient algorithm for approximately solving ODEs. Note that to implement the step, we need to compute the geodesic and compute the probabilities $p(x \stackrel{w}{\rightarrow} y)$ and $p\left(y \stackrel{w^{\prime}}{\rightarrow} x\right)$. From the formula (3.3), we see that they involve the term $d \exp _{x}(w)$. Lemma 34 shows that $d \exp _{x}(w)$ can be computed by a Jacobi field and the latter can be computed by an ODE if it is written in the orthonormal frame systems. Therefore, to implement the geodesic walk, we need to compute geodesic, parallel transport and Jacobi field (See Algorithm 2). All of these are ODEs and can be solved using the collocation method. In the later sections, we will see how to use the collocation method to solve these ODEs in nearly matrix multiplication time for the log barrier.

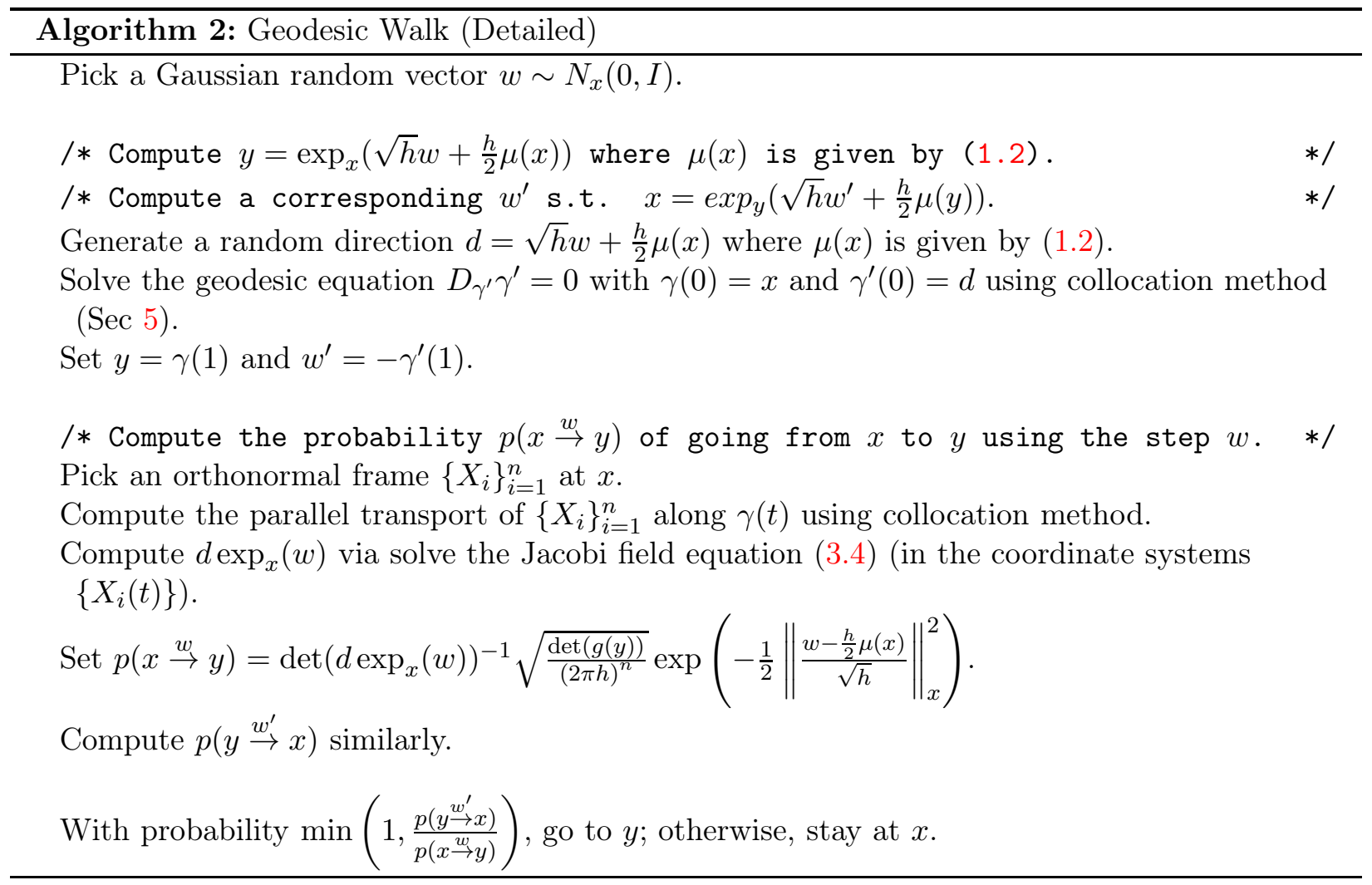




\section{Logarithmic barrier}

For any polytope $M=\{A x>b\}$, the logarithmic barrier function $\phi(x)$ is defined as

$$
\phi(x)=-\sum_{i=1}^{m} \log \left(a_{i}^{T} x-b_{i}\right) .
$$

We denote the Hessian manifold induced by the logarithmic barrier on $M$ by $M_{L}$. The goal of this section is to analyze the geodisic walk on $M_{L}$.

In section 4.1, we give explicit formulas for various Riemannian geometry concepts on $M_{L}$. In Section 4.2, we describe the geodesic walk specialized to $M_{L}$. In Sections 4.3 to 4.7, we bound the parameters required by Theorem 23, resulting in the following theorem.

Theorem 45. The geodesic walk on $M_{L}$ with step size $h=\frac{c}{n^{3 / 4}}$ has mixing time $O\left(m n^{3 / 4}\right)$ for some universal constant $c$.

In later sections, we show how to implement geodesic walk and calculate the rejection probability. To implement these, we apply the techniques developed in Section 5 to solve the corresponding ODEs, after showing that the geodesic, parallel transport and Jacobi field are complex analytic (Section 6.1), for a large radius of convergence (Section 6.1).

Theorem 46. There exists a universal constant $c>0$ s.t. for the standard logarithmic barrier, one step of the geodesic walk with step size $h \leq \frac{c}{\sqrt{n}}$ can be implemented in time $O\left(m n^{\omega-1} \log ^{2}(n)\right)$.

\subsection{Riemannian geometry on $M_{L}\left(G_{2}\right)$}

We use the following definitions throughout this section:

- $A_{x}=S_{x}^{-1} A$.

- $s_{x}=A x-b, S_{x}=\operatorname{Diag}\left(s_{x}\right), s_{x, v}=A_{x} v, S_{x, v}=\operatorname{Diag}\left(A_{x} v\right)$.

- $P_{x}=A_{x}\left(A_{x}^{T} A_{x}\right)^{-1} A_{x}^{T}, \sigma_{x}=\operatorname{Diag}\left(P_{x}\right),\left(P_{x}^{(2)}\right)_{i j}=\left(P_{x}\right)_{i j}^{2}$.

- Gradient of $\phi: \phi_{i}=-\sum_{\ell}\left(e_{\ell}^{T} A_{x} e_{i}\right)$.

- Hessian of $\phi$ and its inverse: $g_{i j}=\phi_{i j}=\sum_{\ell}\left(e_{\ell}^{T} A_{x} e_{i}\right)\left(e_{\ell}^{T} A_{x} e_{j}\right), g^{i j}=e_{i}^{T}\left(A_{x}^{T} A_{x}\right)^{-1} e_{j}$.

- Third derivatives of $\phi: \phi_{i j k}=-2 \sum_{\ell}\left(e_{\ell}^{T} A_{x} e_{i}\right)\left(e_{\ell}^{T} A_{x} e_{j}\right)\left(e_{\ell}^{T} A_{x} e_{k}\right)$.

- For brevity (overloading notation), we define $s_{\gamma^{\prime}}=s_{\gamma, \gamma^{\prime}}, s_{\gamma^{\prime \prime}}=s_{\gamma, \gamma^{\prime \prime}}, S_{\gamma^{\prime}}=S_{\gamma, \gamma^{\prime}}$ and $S_{\gamma^{\prime \prime}}=S_{\gamma, \gamma^{\prime \prime}}$ for a curve $\gamma(t)$.

- Formula for the transition probability:

$$
p\left(x \stackrel{v_{x}}{\rightarrow} y\right)=\sum_{v_{x}: \exp _{x}\left(v_{x}\right)=y} \operatorname{det}\left(d \exp _{x}\left(v_{x}\right)\right)^{-1} \sqrt{\frac{\operatorname{det}\left(A_{y}^{T} A_{y}\right)}{(2 \pi h)^{n}}} \exp \left(-\frac{1}{2 h}\left\|A_{x}\left(v_{x}-\frac{h}{2} \mu(x)\right)\right\|^{2}\right)
$$

where the formula of $\mu(x)$ is given in Lemma 51 . 
In this and subsequent sections, we will frequently use the following elementary calculus facts (using only the chain/product rules and formula for derivative of inverse of a matrix):

$$
\begin{aligned}
\frac{d A_{\gamma}}{d t} & =-S_{\gamma^{\prime}} A_{\gamma}, \\
\frac{d P_{\gamma}}{d t} & =-S_{\gamma^{\prime}} P_{\gamma}-P_{\gamma} S_{\gamma^{\prime}}+2 P_{\gamma} S_{\gamma^{\prime}} P_{\gamma}, \\
\frac{d S_{\gamma^{\prime}}}{d t} & =\operatorname{Diag}\left(-S_{\gamma^{\prime}} A_{\gamma} \gamma^{\prime}+A_{\gamma} \gamma^{\prime \prime}\right)=-S_{\gamma^{\prime}}^{2}+S_{\gamma^{\prime \prime}}, \\
\frac{d \sigma_{\gamma}}{d t} & =\operatorname{Diag}\left(\frac{d P_{\gamma}}{d t}\right) .
\end{aligned}
$$

We also use these matrix inequalities: $\operatorname{Tr}(A B)=\operatorname{Tr}(B A), \operatorname{Tr}(P A P) \leq \operatorname{Tr}(A)$ for any psd ma$\operatorname{trix} A ; \operatorname{Tr}\left(A B A^{T}\right) \leq \operatorname{Tr}\left(A Z A^{T}\right)$ for any $B \preceq Z$; the Cauchy-Schwartz, namely, $\operatorname{Tr}(A B) \leq$ $\operatorname{Tr}\left(A A^{T}\right)^{\frac{1}{2}} \operatorname{Tr}\left(B B^{T}\right)^{\frac{1}{2}}$. We also use $P^{2}=P$ since $P$ is a projection matrix.

Since the Hessian manifold $M_{L}$ is naturally embedded in $\mathbb{R}^{n}$, we identify $T_{x} M_{L}$ by Euclidean coordinates unless otherwise stated. Therefore, we have that

$$
\begin{aligned}
\langle u, v\rangle_{x} & =u^{T} \nabla^{2} \phi(x) v \\
& =u^{T} A_{x}^{T} A_{x} v .
\end{aligned}
$$

Lemma 47. Let $u(t)$ be a vector field defined on a curve $\gamma(t)$ in $M_{L}$. Then, we have that

$$
\nabla_{\gamma^{\prime}} u=\frac{d u}{d t}-\left(A_{\gamma}^{T} A_{\gamma}\right)^{-1} A_{\gamma}^{T} S_{\gamma^{\prime}} s_{\gamma, u} .
$$

In particular, the geodesic equation on $M_{L}$ is given by

$$
\gamma^{\prime \prime}=\left(A_{\gamma}^{T} A_{\gamma}\right)^{-1} A_{\gamma}^{T} s_{\gamma^{\prime}}^{2}
$$

and the equation for parallel transport on a curve $\gamma(t)$ is given by

$$
\frac{d}{d t} v(t)=\left(A_{\gamma}^{T} A_{\gamma}\right)^{-1} A_{\gamma}^{T} S_{\gamma^{\prime}} A_{\gamma} v .
$$

Proof. By Lemma 10, the Christoffel symbols respect to the Euclidean coordinates is given by

$$
\begin{aligned}
\Gamma_{i j}^{k} & =\frac{1}{2} \sum_{l} g^{k l} \phi_{i j l} \\
& =-\sum_{z} \sum_{l} e_{k}^{T}\left(A_{x}^{T} A_{x}\right)^{-1} e_{l}\left(A_{x} e_{i}\right)_{z}\left(A_{x} e_{j}\right)_{z}\left(A_{x} e_{l}\right)_{z} \\
& =-e_{k}^{T}\left(A_{x}^{T} A_{x}\right)^{-1} A_{x}^{T}\left(\left(A_{x} e_{i}\right)\left(A_{x} e_{j}\right)\right) .
\end{aligned}
$$

Recall that the Levi-Civita connection is given by

$$
\nabla_{v} u=\sum_{i k} v_{i} \frac{\partial u_{k}}{\partial x^{i}} e_{k}+\sum_{i j k} v_{i} u_{j} \Gamma_{i j}^{k} e_{k} .
$$

Therefore, we have

$$
\nabla_{v} u=\sum_{i k} v^{i} \frac{\partial u^{k}}{\partial x^{i}} e_{k}-\left(A_{x}^{T} A_{x}\right)^{-1} A_{x}^{T} S_{x, v} s_{x, u} .
$$

Since $U$ is a vector field defined on a curve $\gamma(t)$, we have that $\sum_{i k} \gamma_{i}^{\prime} \frac{\partial u_{k}}{\partial x^{i}} e_{k}=\frac{d u}{d t}$.

The geodesic equation follows from $\nabla_{\gamma^{\prime}} \gamma^{\prime}=0$ and the parallel transport equation follows from $\nabla_{\gamma^{\prime}} v=0$. 
Lemma 48. Given $u, v, w, z \in T_{x} M_{L}$, the Riemann Curvature Tensor is given by

$$
\begin{aligned}
\langle R(u, v) w, z\rangle & =\left(s_{x, u} s_{x, w}\right)^{T} P_{x}\left(s_{x, v} s_{x, z}\right)-\left(s_{x, u} s_{x, z}\right)^{T} P_{x}\left(s_{x, v} s_{x, w}\right) \\
R(u, v) w & =\left(A_{x}^{T} A_{x}\right)^{-1} A_{x}^{T}\left(S_{x, v} P_{x} S_{x, w}-\operatorname{diag}\left(P_{x} s_{x, v} s_{x, w}\right)\right) A_{x} u
\end{aligned}
$$

and the Ricci curvature is given by

$$
\operatorname{Ric}(u)=s_{x, u}^{T} P_{x}^{(2)} s_{x, u}-\sigma_{x}^{T} P_{x} s_{x, u}^{2} .
$$

Proof. By Lemma 10, we have that

$$
\begin{aligned}
\langle R(u, v) w, z\rangle & =\frac{1}{4} \sum_{p q i j l k} g^{p q}\left(\phi_{j k p} \phi_{i l q}-\phi_{i k p} \phi_{j l q}\right) u_{i} v_{j} w_{l} z_{k} \\
& =\sum_{p q} g^{p q}\left(e_{p}^{T} A_{x}^{T}\left(s_{x, v} s_{x, z}\right) e_{q}^{T} A_{x}^{T}\left(s_{x, u} s_{x, w}\right)-e_{p}^{T} A_{x}^{T}\left(s_{x, u} s_{x, z}\right) e_{q}^{T} A_{x}^{T}\left(s_{x, v} s_{x, w}\right)\right) \\
& =\left(s_{x, u} s_{x, w}\right)^{T} A_{x}\left(A_{x}^{T} A_{x}\right)^{-1} A_{x}^{T}\left(s_{x, v} s_{x, z}\right)-\left(s_{x, u} s_{x, z}\right)^{T} A_{x}\left(A_{x}^{T} A_{x}\right)^{-1} A_{x}^{T}\left(s_{x, v} s_{x, w}\right) .
\end{aligned}
$$

Rewriting it, we have that

$$
\begin{aligned}
\langle R(u, v) w, z\rangle & =s_{x, u} S_{x, w} P_{x} S_{x, v} s_{x, z}-\left(s_{x, u} s_{x, z}\right)^{T} P_{x}\left(s_{x, v} s_{x, w}\right) \\
& =z^{T} A_{x}^{T} S_{x, w} P_{x} S_{x, v} A_{x} u-z^{T} A_{x}^{T} \operatorname{diag}\left(P_{x} s_{x, v} s_{x, w}\right) A_{x} u \\
& =z^{T} A_{x}^{T}\left(S_{x, w} P_{x} S_{x, v}-\operatorname{diag}\left(P_{x} s_{x, v} s_{x, w}\right)\right) A_{x} u
\end{aligned}
$$

Since $\langle\alpha, \beta\rangle=\alpha^{T} A_{x}^{T} A_{x} \beta$, we have that

$$
R(u, v) w=\left(A_{x}^{T} A_{x}\right)^{-1} A_{x}^{T}\left(A_{x}^{T} S_{x, w} P_{x} S_{x, v}-\operatorname{diag}\left(P_{x} s_{x, v} s_{x, w}\right)\right) A_{x} u .
$$

For the Ricci curvature, we have that

$$
\begin{aligned}
\operatorname{Ric}(u) & =\sum_{j l} g^{j l}\left\langle R\left(u, \frac{\partial}{\partial x^{j}}\right), \frac{\partial}{\partial x^{l}}, u\right\rangle \\
& =\sum_{j l} g^{j l}\left(\left(s_{x, u} s_{x, e_{l}}\right)^{T} P_{x}\left(s_{x, e_{j}} s_{x, u}\right)-\left(s_{x, u}^{2}\right)^{T} P_{x}\left(s_{x, e_{j}} s_{x, e_{l}}\right)\right) \\
& =\sum_{j l} g^{j l} e_{l}^{T} A_{x}^{T}\left(S_{x, u} P_{x} S_{x, u}-\operatorname{diag}\left(P_{x} s_{x, u}^{2}\right)\right) A_{x} e_{j} \\
& =\operatorname{Tr}\left(\left(A_{x}^{T} A_{x}\right)^{-1} A_{x}^{T}\left(S_{x, u} P_{x} S_{x, u}-\operatorname{diag}\left(P_{x} s_{x, u}^{2}\right)\right) A_{x}\right) \\
& =\operatorname{Tr} P_{x} S_{x, u} P_{x} S_{x, u}-\operatorname{Tr} P_{x} \operatorname{diag}\left(P_{x} s_{x, u}^{2}\right) \\
& =s_{x, u}^{T} P_{x}^{(2)} s_{x, u}-\sigma_{x}^{T} P_{x} s_{x, u}^{2} .
\end{aligned}
$$

Lemma 49. Given a geodesic $\gamma(t)$ on $M_{L}$ and an orthogonal frame $\left\{x_{i}\right\}_{i=1}^{n}$ on $\gamma(t)$. The Jacobi field equation (in the orthogonal frame coordinates) is given by

$$
\frac{d^{2} u}{d t^{2}}+X^{-1}\left(A_{\gamma}^{T} A_{\gamma}\right)^{-1}\left(A_{\gamma}^{T} S_{\gamma^{\prime}} P_{\gamma} S_{\gamma^{\prime}} A_{\gamma}-A_{\gamma}^{T} \operatorname{Diag}\left(P_{\gamma} s_{\gamma^{\prime}}^{2}\right) A_{\gamma}\right) X u=0
$$

where $X(t)=\left[x_{1}(t), x_{2}(t), \cdots, x_{n}(t)\right]$. 
Proof. The equation for Jacobi field along is

$$
D_{t}^{2} v+R\left(v, \gamma^{\prime}\right) \gamma^{\prime}=0
$$

By Lemma 48, under Euclidean coordinates, we have

$$
D_{t}^{2} v+\left(A_{\gamma}^{T} A_{\gamma}\right)^{-1}\left(A_{\gamma}^{T} S_{\gamma^{\prime}} P_{\gamma} S_{\gamma^{\prime}} A_{\gamma}-A_{\gamma}^{T} \operatorname{diag}\left(P_{\gamma} s_{\gamma^{\prime}}^{2}\right) A_{\gamma}\right) v=0
$$

We write $v$ in terms of the orthogonal frame, namely, $v(t)=X(t) u(t)$ where $u(t) \in \mathbb{R}^{n}$. Then, we have that $D_{t}^{2} v=X(t) \frac{d^{2} u(t)}{d t^{2}}$. Hence, under the orthogonal frame coordinate, we have that

$$
\frac{d^{2} u}{d t^{2}}+X^{-1}\left(A_{\gamma}^{T} A_{\gamma}\right)^{-1}\left(A_{\gamma}^{T} S_{\gamma^{\prime}} P_{\gamma} S_{\gamma^{\prime}} A_{\gamma}-A_{\gamma}^{T} \operatorname{Diag}\left(P_{\gamma} s_{\gamma^{\prime}}^{2}\right) A_{\gamma}\right) X u=0 .
$$

Now, we show the relation between the distance induced by the metric and the Hilbert metric.

Lemma 50. For any $x, y \in M_{L}$, we have that

$$
\frac{d(x, y)}{d_{H}(x, y)} \leq \sqrt{m}
$$

Hence, we have that $G_{2} \leq \sqrt{m}$.

Proof. First, we note that it suffices to prove that $\frac{d(x, y)}{d_{H}(x, y)} \leq(1+O(\varepsilon)) \sqrt{m}$ for any $x, y \in M_{L}$ with $d(x, y) \leq \varepsilon$. Then, one can run a limiting argument as follows. Let $x_{t}=t \cdot x+(1-t) \cdot y$, then we have that

$$
d_{H}(x, y)=\lim _{n \rightarrow \infty} \sum_{i=0}^{n-1} d_{H}\left(x_{k / n}, x_{(k+1) / n}\right) .
$$

Since $d_{H}\left(x_{k / n}, x_{(k+1) / n}\right)=O_{x, y}\left(\frac{1}{n}\right)$, we have that

$$
\begin{aligned}
d_{H}(x, y) & =\lim _{n \rightarrow \infty} \sum_{i=0}^{n-1} d_{H}\left(x_{k / n}, x_{(k+1) / n}\right) \\
& \geq \lim _{n \rightarrow \infty} \frac{\left(1-O_{x, y}\left(\frac{1}{n}\right)\right)}{\sqrt{m}} \sum_{i=0}^{n-1} d\left(x_{k / n}, x_{(k+1) / n}\right) \\
& =\frac{1}{\sqrt{m}} \lim _{n \rightarrow \infty} \sum_{i=0}^{n-1} d\left(x_{k / n}, x_{(k+1) / n}\right) \\
& \geq \frac{1}{\sqrt{m}} d(x, y)=\frac{d(x, y)}{\sqrt{m}}
\end{aligned}
$$

Now, we can assume $d(x, y) \leq \varepsilon$. Let $p$ and $q$ are on the boundary of $M_{L}$ such that $p, x, y, q$ are on the straight line $\overline{x y}$ and are in order. Without loss of generality, we assume $p$ is closer to $x$. Then, we have that $p \in M_{L} \cap\left(x-M_{L}\right)$, equivalently, we have that $\left|a_{i}^{T} p-a_{i}^{T} x\right| \leq a_{i}^{T} x-b_{i}$ for all $i$ and hence $\left\|A_{x}(p-x)\right\|_{\infty} \leq 1$. Therefore, we have that

$$
\left\|A_{x}(p-x)\right\|_{2} \leq \sqrt{m}\left\|A_{x}(p-x)\right\|_{\infty} \leq \sqrt{m} .
$$


Since $p, x, y$ are on the same line, we have that

$$
\frac{\|x-y\|_{2}\|p-q\|_{2}}{\|p-x\|_{2}\|y-q\|_{2}} \geq \frac{\|x-y\|_{2}}{\|p-x\|_{2}}=\frac{\left\|A_{x}(x-y)\right\|_{2}}{\left\|A_{x}(p-x)\right\|_{2}} \geq \frac{\left\|A_{x}(x-y)\right\|_{2}}{\sqrt{m}} .
$$

Since $d(x, y)<\varepsilon$, Lemma 3.1 in [31] shows that

$$
d(x, y) \leq-\log \left(1-\left\|A_{x}(x-y)\right\|_{2}\right) .
$$

Hence, we have that $\left\|A_{x}(x-y)\right\|_{2} \geq(1-O(\varepsilon)) d(x, y)$ and hence

$$
d_{H}(x, y)=\frac{\|x-y\|_{2}\|p-q\|_{2}}{\|p-x\|_{2}\|y-q\|_{2}} \geq(1-O(\varepsilon)) \frac{d(x, y)}{\sqrt{m}} .
$$

\subsection{Geodesic walk on $M_{L}$}

Recall that the geodesic walk is given by

$$
x^{(\text {new })}=\exp _{x}\left(\sqrt{h} w+\frac{h}{2} \mu(x)\right)
$$

where $w \sim N_{x}(0, I)$. In many proofs in this section, we consider the geodesic $\gamma$ from $x$ with the initial velocity

$$
\gamma^{\prime}(0)=\frac{w}{\sqrt{n}}+\frac{1}{2} \sqrt{\frac{h}{n}} \mu(x) .
$$

The scaling is to make the speed of geodesic $\gamma$ close to one. Since $w$ is a Gaussian vector, we have that $0.9 \leq\left\|\gamma^{\prime}(0)\right\|_{\gamma(0)} \leq 1.1$ with high probability. Due to this rescaling, the geodesic is defined from 0 to $\ell=\sqrt{n h}$.

We often work in Euclidean coordinates. In this case, the geodesic walk is given by the following formula.

Lemma 51. Given $x \in M_{L}$ and step size $h>0$, one step of the geodesic walk starting at $x$ in Euclidean coordinates is given by the solution $\gamma(\ell)$ of the following geodesic equation

$$
\begin{aligned}
\gamma^{\prime \prime}(t) & =\left(A_{\gamma}^{T} A_{\gamma}\right)^{-1} A_{\gamma}^{T} s_{\gamma^{\prime}}^{2} \text { for } 0 \leq t \leq \ell \\
\gamma^{\prime}(0) & =\frac{w}{\sqrt{n}}+\frac{1}{2} \sqrt{\frac{h}{n}} \mu(x) \\
\gamma(0) & =x
\end{aligned}
$$

where $\ell=\sqrt{n h}, w \sim N\left(0,\left(A_{x}^{T} A_{x}\right)^{-1}\right)$ and $\mu(x)=\left(A_{x}^{T} A_{x}\right)^{-1} A_{x}^{T} \sigma_{x}$. 
Proof. The geodesic equation is given by Lemma 47. From (1.2), the drift term is given by

$$
\begin{aligned}
\mu_{i}(x) & =\frac{1}{2} \sum_{j=1}^{n} \frac{\partial}{\partial x_{j}}\left(\left(\nabla^{2} \phi\left(X_{t}\right)\right)^{-1}\right)_{i j}=\frac{1}{2} \sum_{j=1}^{n} \frac{\partial}{\partial x_{j}}\left(\left(A_{x}^{T} A_{x}\right)^{-1}\right)_{i j} \\
& =\sum_{j} e_{i}^{T}\left(A_{x}^{T} A_{x}\right)^{-1} A_{x}^{T} S_{x, e_{j}} A_{x}\left(A_{x}^{T} A_{x}\right)^{-1} e_{j} \\
& =\sum_{j} \sum_{k} V_{i k} e_{k}^{T} A_{x} e_{j} V_{j k} \quad \text { where } V=\left(A_{x}^{T} A_{x}\right)^{-1} A_{x}^{T} \\
& =\sum_{k} V_{i k} e_{k}^{T} A_{x} \sum_{j} e_{j} e_{j}^{T}\left(A_{x}^{T} A_{x}\right)^{-1} A_{x}^{T} e_{k} \\
& =\sum_{k} V_{i k} e_{k}^{T} A_{x}\left(A_{x}^{T} A_{x}\right)^{-1} A_{x}^{T} e_{k} \\
& =V_{i} \sigma_{x}=e_{i}^{T}\left(A_{x}^{T} A_{x}\right)^{-1} A_{x}^{T} \sigma_{x} .
\end{aligned}
$$

\subsection{Randomness and stability of the geodesic $\left(V_{0}\right)$}

Many parameters of a Hessian manifold relates to how fast a geodesic approaches the boundary of the polytope. Since the initial velocity of the geodesic consists mainly the Gaussian part (plus a small drift term), one can imagine that $\left\|s_{\gamma^{\prime}(0)}\right\|_{\infty}=O\left(\frac{1}{\sqrt{n}}\right)\left\|s_{\gamma^{\prime}(0)}\right\|_{2}$, namely, the geodesic initial approaches/leaves every facet of the polytope in roughly same slow pace. If this holds on the whole geodesic, this would allowed us to give very tight bounds on various parameters. Although we are not able to prove that $\left\|s_{\gamma^{\prime}(t)}\right\|_{\infty}$ is stable throughout $0 \leq t \leq \ell$, we can show that $\left\|s_{\gamma^{\prime}(t)}\right\|_{4}$ is stable and that allows us to give a good bound on $\left\|s_{\gamma^{\prime}(t)}\right\|_{\infty}$.

Throughout this section, we only use the randomness of geodesic to prove that both $\left\|s_{\gamma^{\prime}(t)}\right\|_{4}$ and $\left\|s_{\gamma^{\prime}(t)}\right\|_{\infty}$ is small with high probability. Since $\left\|s_{\gamma^{\prime}(t)}\right\|_{4}=O\left(n^{-1 / 4}\right)$ and $\left\|s_{\gamma^{\prime}(t)}\right\|_{\infty}=O\left(\sqrt{\frac{\log n}{n}}+\sqrt{h}\right)$ with high probability (Lemma 53), we define

$$
V(\gamma) \stackrel{\text { def }}{=} \max _{0 \leq t \leq \ell}\left(\frac{\left\|s_{\gamma^{\prime}(t)}\right\|_{4}}{n^{-1 / 4}}+\frac{\left\|s_{\gamma^{\prime}(t)}\right\|_{\infty}}{\sqrt{\frac{\log n}{n}}+\sqrt{h}}\right)
$$

to capture this randomness involves in generating the geodesic walk. This allows us to perturb the geodesic (Lemma 44) without worrying about the dependence on randomness.

Here, we first prove the the geodesic is stable in $L_{4}$ norm and hence $V(\gamma)$ can be simply approximated by $\left\|s_{\gamma^{\prime}(0)}\right\|_{4}$ and $\left\|s_{\gamma^{\prime}(0)}\right\|_{\infty}$.

Lemma 52. Let $\gamma$ be a geodesic in $M_{L}$ starting at $x$. Let $v_{4}=\left\|s_{\gamma^{\prime}(0)}\right\|_{4}$. Then, for $0 \leq t \leq \frac{1}{10 v_{4}}$, we have that

1. $\left\|s_{\gamma^{\prime}(t)}\right\|_{4} \leq 1.25 v_{4}$.

2. $\left\|\gamma^{\prime \prime}(t)\right\|_{\gamma(t)}^{2} \leq 3 v_{4}^{4}$. 
Proof. Let $u(t)=\left\|s_{\gamma^{\prime}(t)}\right\|_{4}$. Then, we have

$$
\begin{aligned}
\frac{d u}{d t} & \leq\left\|\frac{d}{d t}\left(A_{\gamma} \gamma^{\prime}\right)\right\|_{4} \\
& =\left\|A_{\gamma} \gamma^{\prime \prime}-\left(A_{\gamma} \gamma^{\prime}\right)^{2}\right\|_{4} \\
& \leq\left\|A_{\gamma} \gamma^{\prime \prime}\right\|_{4}+u^{2}(t) .
\end{aligned}
$$

Under the Euclidean coordinates, the geodesic equation is given by

$$
\gamma^{\prime \prime}=\left(A_{\gamma}^{T} A_{\gamma}\right)^{-1} A_{\gamma}^{T} s_{\gamma^{\prime}}^{2}
$$

Hence, we have that

$$
\begin{aligned}
\left\|\gamma^{\prime \prime}\right\|_{\gamma}^{2} & =\left(s_{\gamma^{\prime}}^{2}\right)^{T} A_{\gamma}\left(A_{\gamma}^{T} A_{\gamma}\right)^{-1}\left(A_{\gamma}^{T} A_{\gamma}\right)\left(A_{\gamma}^{T} A_{\gamma}\right)^{-1} A_{\gamma}^{T} s_{\gamma^{\prime}}^{2} \\
& \leq \sum_{i}\left(s_{\gamma^{\prime}}^{4}\right)_{i}=u^{4}(t) .
\end{aligned}
$$

Therefore, we have

$$
\left\|A_{\gamma} \gamma^{\prime \prime}\right\|_{4} \leq\left\|A_{\gamma} \gamma^{\prime \prime}\right\|_{2} \leq u^{2}(t) .
$$

Plugging it into (4.3), we have that

$$
\frac{d u}{d t} \leq u^{2}(t)+u^{2}(t) \leq 2 u^{2}(t) .
$$

Since $u(0)=v_{4}$, for all $t \geq 0$, we have

$$
u(t) \leq \frac{v_{4}}{1-2 v_{4} t} .
$$

For $0 \leq t \leq \frac{1}{10 v_{4}}$, we have $u(t) \leq 1.25 v_{4}$ and this gives the first inequality. Using (4.4), we get the second inequality.

Using this, we prove that $V(\gamma)$ is small with high probability.

Lemma 53. Assume that $h \leq \frac{1}{1000 \sqrt{n}}$. We have that

$$
P(V(\gamma) \leq 24) \geq 1-\frac{3}{n}
$$

where $\gamma$ is generated from geodesic walk.

Proof. From the definition of the geodesic walk (Lemma 51), we have that

$$
\gamma^{\prime}(0)=u+v
$$

where $u \sim N\left(0, \frac{1}{n}\left(A_{\gamma}^{T} A_{\gamma}\right)^{-1}\right)$ and $v=\frac{1}{2} \sqrt{\frac{h}{n}}\left(A_{\gamma}^{T} A_{\gamma}\right)^{-1} A_{\gamma}^{T} \sigma_{\gamma}$. Therefore, we have

$$
\begin{aligned}
\left\|A_{\gamma} \gamma^{\prime}(0)\right\|_{4} & \leq\left\|A_{\gamma} u\right\|_{4}+\left\|\frac{1}{2} \sqrt{\frac{h}{n}} A_{\gamma}\left(A_{\gamma}^{T} A_{\gamma}\right)^{-1} A_{\gamma}^{T} \sigma_{\gamma}\right\|_{2} \\
& =\left\|A_{\gamma} u\right\|_{4}+\frac{1}{2} \sqrt{\frac{h}{n}} \sqrt{\sigma_{\gamma}^{T} A_{\gamma}\left(A_{\gamma}^{T} A_{\gamma}\right)^{-1} A_{\gamma}^{T} \sigma_{\gamma}} .
\end{aligned}
$$


Note that $A_{\gamma} u=B x$ where $B=\frac{1}{\sqrt{n}} A_{\gamma}\left(A_{\gamma}^{T} A_{\gamma}\right)^{-1 / 2}$ and $x \sim N(0, I)$. Since $\sum_{i}\left\|e_{i}^{T} B\right\|_{2}^{4}=$ $\frac{1}{n^{2}} \sum_{i}\left(\sigma_{\gamma}\right)_{i}^{2} \leq \frac{1}{n}$ and $\|B\|_{2 \rightarrow 4} \leq\|B\|_{2 \rightarrow 2}=\frac{1}{\sqrt{n}}$. Hence, Lemma 90 shows that

$$
P\left(\left\|A_{\gamma} u\right\|_{4}^{4} \leq\left(\left(\frac{3}{n}\right)^{1 / 4}+\frac{t}{\sqrt{n}}\right)^{4}\right) \leq 1-\exp \left(-\frac{t^{2}}{2}\right) .
$$

Using our assumption on $h$, we have that

$$
v_{4} \stackrel{\text { def }}{=}\left\|A_{\gamma} \gamma^{\prime}(0)\right\|_{4} \leq \frac{3^{1 / 4}+\sqrt{2}}{n^{1 / 4}}+\frac{1}{2} \sqrt{h} \leq \frac{3}{n^{1 / 4}}
$$

with probability at least $1-\exp (-\sqrt{n})$. Now, we apply Lemma 52 to get that

$$
\left\|s_{\gamma^{\prime}(t)}\right\|_{4} \leq 1.25 v_{4} \leq \frac{4}{n^{1 / 4}}
$$

for all $0 \leq t \leq \ell \leq \frac{1}{10 v_{4}}$

Next, we estimate $\left\|s_{\gamma^{\prime}(t)}\right\|_{\infty}$. Since $e_{i}^{T} A_{\gamma} u=e_{i}^{T} B x \sim N\left(0, \frac{\sigma_{i}}{n}\right)$, we have

$$
P_{u}\left(\left|e_{i}^{T} A_{\gamma} u\right| \geq \sqrt{\frac{\sigma_{i}}{n}} t\right) \leq 2 \exp \left(-\frac{t^{2}}{2}\right) .
$$

Hence, we have that

$$
P_{u}\left(\left\|A_{\gamma} u\right\|_{\infty} \geq 2 \sqrt{\frac{\log n}{n}}\right) \leq 2 \sum_{i} \exp \left(-\frac{2 \log n}{\sigma_{i}}\right)
$$

Since $\sum_{i} \exp \left(-\frac{2 \log n}{\sigma_{i}}\right)$ is concave, the maximum of $\sum_{i} \exp \left(-\frac{\log n}{\sigma_{i}}\right)$ on the feasible set $\{0 \leq \sigma \leq$ $\left.1, \sum \sigma_{i}=n\right\}$ occurs on its vertices. Hence, we have that

$$
P_{u}\left(\left\|A_{\gamma} u\right\|_{\infty} \geq 2 \sqrt{\frac{\log n}{n}}\right) \leq 2 n \exp (-2 \log n)=\frac{2}{n} .
$$

Therefore, with probability $1-\frac{2}{n}$, we have that

$$
\begin{aligned}
\left\|A_{\gamma} \gamma^{\prime}(0)\right\|_{\infty} & \leq\left\|A_{\gamma} u\right\|_{\infty}+\left\|\frac{1}{2} \sqrt{\frac{h}{n}} A_{\gamma}\left(A_{\gamma}^{T} A_{\gamma}\right)^{-1} A_{\gamma}^{T} \sigma_{\gamma}\right\|_{\infty} \\
& \leq 2 \sqrt{\frac{\log n}{n}}+\frac{1}{2} \sqrt{\frac{h}{n}} \sqrt{\sigma_{\gamma}^{T} A_{\gamma}\left(A_{\gamma}^{T} A_{\gamma}\right)^{-1} A_{\gamma}^{T} \sigma_{\gamma}} \\
& \leq 2 \sqrt{\frac{\log n}{n}}+\frac{1}{2} \sqrt{h} .
\end{aligned}
$$

Lemma 52 shows that $\left\|A_{\gamma} \gamma^{\prime \prime}\right\|_{\infty} \leq\left\|\gamma^{\prime \prime}\right\|_{\gamma(t)} \leq \sqrt{3} v_{4}^{2} \leq 16 n^{-1 / 2}$. Hence, for any $0 \leq t \leq \ell$, we have that

$$
\begin{aligned}
\left\|s_{\gamma^{\prime}(t)}\right\|_{\infty} & \leq\left\|A_{\gamma} \gamma^{\prime}(0)\right\|_{\infty}+\int_{0}^{\ell}\left\|A_{\gamma(t)} \gamma^{\prime \prime}(t)\right\|_{\infty} d t \\
& \leq 2 \sqrt{\frac{\log n}{n}}+\frac{1}{2} \sqrt{h}+16 \ell n^{-1 / 2} \\
& =20\left(\sqrt{\frac{\log n}{n}}+\sqrt{h}\right) .
\end{aligned}
$$


Combining with our estimate on $\left\|s_{\gamma^{\prime}(t)}\right\|_{4}$, we have that

$$
P\left(\frac{\left\|s_{\gamma^{\prime}(t)}\right\|_{4}}{n^{-1 / 4}}+\frac{\left\|s_{\gamma^{\prime}(t)}\right\|_{\infty}}{\sqrt{\frac{\log n}{n}}+\sqrt{h}} \geq 24\right) \leq e^{-\sqrt{n}}+\frac{2}{n} \leq \frac{3}{n} .
$$

Due to this lemma, we set

$$
V_{0}=48 .
$$

Here, we collect some simple consequences of small $V(\gamma)$ that we will use through this section.

Lemma 54. Given a geodesic $\gamma$ on $M_{L}$ with $V(\gamma) \leq V_{0}$. For any $0 \leq t \leq \ell$,

1. $\left\|A_{\gamma} \gamma^{\prime}(t)\right\|_{4} \leq 48 n^{-1 / 4}$.

2. $\left\|A_{\gamma} \gamma^{\prime}(t)\right\|_{\infty} \leq 48\left(\sqrt{\frac{\log n}{n}}+\sqrt{h}\right)$.

3. $\left\|A_{\gamma} \gamma^{\prime}(t)\right\|_{2} \leq 48$.

4. $\left\|\gamma^{\prime \prime}(t)\right\|_{\gamma}^{2} \leq 10^{8} n^{-1}$.

Proof. The first two inequality simply follows from the definition of $V(\gamma)$. The third inequality comes from the calculation

$$
\left\|A_{\gamma} \gamma^{\prime}\right\|_{2} \leq n^{1 / 4}\left\|A_{\gamma} \gamma^{\prime}\right\|_{4} \leq 48
$$

Since $\left\|A_{\gamma} \gamma^{\prime}(0)\right\|_{4} \leq 48 n^{-1 / 4}$, Lemma 52 shows the last inequality.

\subsection{Stability of Drift $\left(D_{0}, D_{1}\right.$ and $\left.D_{2}\right)$}

Lemma 55. For any $x \in M_{L}$, we have that $\|\mu(x)\|_{x}^{2} \leq n$. Hence, $D_{0} \leq \sqrt{n}$.

Proof. We have

$$
\|\mu(x)\|_{x}^{2}=\sigma_{x}^{T} A_{x}\left(A_{x}^{T} A_{x}\right)^{-1} A_{x} \sigma_{x} \leq \sum_{i}\left(\sigma_{x}\right)_{i}^{2} \leq n .
$$

Lemma 56. Let $\gamma(t)$ be a geodesic on $M_{L}$ with $V(\gamma) \leq V_{0}$. Then, we have that

$$
D_{1}=\sup _{0 \leq t \leq \ell}\left|\frac{d}{d t}\|\mu(\gamma(t))\|^{2}\right|=O(n \sqrt{h}+\sqrt{n \log n}) .
$$

Proof. Note that

$$
\begin{aligned}
\|\mu(\gamma(t))\|_{\gamma}^{2} & =\sigma_{\gamma}^{T} A_{\gamma}\left(A_{\gamma}^{T} A_{\gamma}\right)^{-1} A_{\gamma} \sigma_{\gamma} \\
& =1^{T} \operatorname{Diag}\left(P_{\gamma}\right) P_{\gamma} \operatorname{Diag}\left(P_{\gamma}\right) 1
\end{aligned}
$$


Using $\frac{d}{d t} P_{\gamma}=-S_{\gamma^{\prime}} P_{\gamma}-P_{\gamma} S_{\gamma^{\prime}}+2 P_{\gamma} S_{\gamma^{\prime}} P_{\gamma}$, we have

$$
\begin{aligned}
\frac{d}{d t}\|\mu(\gamma(t))\|_{\gamma}^{2}= & -2 \cdot 1^{T} \operatorname{Diag}\left(S_{\gamma^{\prime}} P_{\gamma}\right) P_{\gamma} \operatorname{Diag}\left(P_{\gamma}\right) 1 \\
& -2 \cdot 1^{T} \operatorname{Diag}\left(P_{\gamma} S_{\gamma^{\prime}}\right) P_{\gamma} \operatorname{Diag}\left(P_{\gamma}\right) 1 \\
& +4 \cdot 1^{T} \operatorname{Diag}\left(P_{\gamma} S_{\gamma^{\prime}} P_{\gamma}\right) P_{\gamma} \operatorname{Diag}\left(P_{\gamma}\right) 1 \\
& -1^{T} \operatorname{Diag}\left(P_{\gamma}\right) S_{\gamma^{\prime}} P_{\gamma} \operatorname{Diag}\left(P_{\gamma}\right) 1 \\
& -1^{T} \operatorname{Diag}\left(P_{\gamma}\right) P_{\gamma} S_{\gamma^{\prime}} \operatorname{Diag}\left(P_{\gamma}\right) 1 \\
& +2 \cdot 1^{T} \operatorname{Diag}\left(P_{\gamma}\right) P_{\gamma} S_{\gamma^{\prime}} P_{\gamma} \operatorname{Diag}\left(P_{\gamma}\right) 1 \\
= & -2 \cdot 1^{T} \operatorname{Diag}\left(S_{\gamma^{\prime}} P_{\gamma}\right) P_{\gamma} \operatorname{Diag}\left(P_{\gamma}\right) 1 \\
& -4 \cdot 1^{T} \operatorname{Diag}\left(P_{\gamma}\right) S_{\gamma^{\prime}} P_{\gamma} \operatorname{Diag}\left(P_{\gamma}\right) 1 \\
& +4 \cdot 1^{T} \operatorname{Diag}\left(P_{\gamma} S_{\gamma^{\prime}} P_{\gamma}\right) P_{\gamma} \operatorname{Diag}\left(P_{\gamma}\right) 1 \\
& +2 \cdot 1^{T} \operatorname{Diag}\left(P_{\gamma}\right) P_{\gamma} S_{\gamma^{\prime}} P_{\gamma} \operatorname{Diag}\left(P_{\gamma}\right) 1 .
\end{aligned}
$$

Now, we bound these 4 terms separately. Note that

$$
\begin{aligned}
\left|1^{T} \operatorname{Diag}\left(S_{\gamma^{\prime}} P_{\gamma}\right) P_{\gamma} \operatorname{Diag}\left(P_{\gamma}\right) 1\right| & \leq \sqrt{1^{T} \operatorname{Diag}\left(S_{\gamma^{\prime}} P_{\gamma}\right) P_{\gamma} \operatorname{Diag}\left(S_{\gamma^{\prime}} P_{\gamma}\right) 1} \sqrt{1^{T} \operatorname{Diag}\left(P_{\gamma}\right) P_{\gamma} \operatorname{Diag}\left(P_{\gamma}\right) 1} \\
& \leq \sqrt{\sum_{i}\left(\sigma_{\gamma} s_{\gamma^{\prime}}\right)_{i}^{2}} \sqrt{\sum_{i}\left(\sigma_{\gamma}\right)_{i}^{2}} \leq\left\|\gamma^{\prime}\right\|_{\gamma} \sqrt{n}, \\
\left|1^{T} \operatorname{Diag}\left(P_{\gamma}\right) S_{\gamma^{\prime}} P_{\gamma} \operatorname{Diag}\left(P_{\gamma}\right) 1\right| & \leq \sqrt{1^{T} \operatorname{Diag}\left(P_{\gamma}\right) S_{\gamma^{\prime}} P_{\gamma} S_{\gamma^{\prime}} \operatorname{Diag}\left(P_{\gamma}\right) 1} \sqrt{1^{T} \operatorname{Diag}\left(P_{\gamma}\right) P_{\gamma} \operatorname{Diag}\left(P_{\gamma}\right) 1} \\
& \leq \sqrt{\sum_{i}\left(\sigma_{\gamma} s_{\gamma^{\prime}}\right)_{i}^{2}} \sqrt{\sum_{i}\left(\sigma_{\gamma}\right)_{i}^{2}} \leq\left\|\gamma^{\prime}\right\|_{\gamma} \sqrt{n},
\end{aligned}
$$

$\left|1^{T} \operatorname{Diag}\left(P_{\gamma} S_{\gamma^{\prime}} P_{\gamma}\right) P_{\gamma} \operatorname{Diag}\left(P_{\gamma}\right) 1\right| \leq \sqrt{1^{T} \operatorname{Diag}\left(P_{\gamma} S_{\gamma^{\prime}} P_{\gamma}\right) P_{\gamma} \operatorname{Diag}\left(P_{\gamma} S_{\gamma^{\prime}} P_{\gamma}\right) 1} \sqrt{1^{T} \operatorname{Diag}\left(P_{\gamma}\right) P_{\gamma} \operatorname{Diag}\left(P_{\gamma}\right) 1}$

$$
\begin{aligned}
& \leq \sqrt{\sum_{i}\left(P_{\gamma} S_{\gamma^{\prime}} P_{\gamma}\right)_{i i}^{2}} \sqrt{\sum_{i}\left(\sigma_{\gamma}\right)_{i}^{2}} \\
& \leq\left\|S_{\gamma^{\prime}}\right\|_{\infty} \sum_{i}\left(\sigma_{\gamma}\right)_{i}^{2} \leq\left\|S_{\gamma^{\prime}}\right\|_{\infty} n,
\end{aligned}
$$

$$
\begin{aligned}
1^{T} \operatorname{Diag}\left(P_{\gamma}\right) P_{\gamma} S_{\gamma^{\prime}} P_{\gamma} \operatorname{Diag}\left(P_{\gamma}\right) 1 & \leq\left\|S_{\gamma^{\prime}}\right\|_{\infty} 1^{T} \operatorname{Diag}\left(P_{\gamma}\right) P_{\gamma} P_{\gamma} \operatorname{Diag}\left(P_{\gamma}\right) 1 \\
& \leq\left\|S_{\gamma^{\prime}}\right\|_{\infty} \sum_{i}\left(\sigma_{\gamma}\right)_{i}^{2} \leq\left\|S_{\gamma^{\prime}}\right\|_{\infty} n
\end{aligned}
$$

Since $\left\|\gamma^{\prime}\right\|_{\gamma} \leq 48$ and $\left\|A_{\gamma} \gamma^{\prime}\right\|_{\infty} \leq 48\left(\sqrt{\frac{\log n}{n}}+\sqrt{h}\right)$ (Lemma 54), we have

$$
D_{1}=\sup \left|\frac{d}{d t}\|\mu(\gamma(t))\|^{2}\right| \leq 288 \sqrt{n}+288 n\left(\sqrt{\frac{\log n}{n}}+\sqrt{h}\right) \leq 576 \sqrt{n \log n}+288 n \sqrt{h}
$$


Lemma 57. Given $x \in M_{L}$ and any curve $c(t)$ starting at $x$ with unit speed. We have that

$$
\left\|D_{t} \mu(c(t))\right\|=O(\sqrt{n}) .
$$

Hence, $D_{2}=O(\sqrt{n})$.

Proof. By Lemma 47, we have that

$$
D_{t} \mu(c(t))=\frac{d \mu(c)}{d t}-\left(A_{c}^{T} A_{c}\right)^{-1} A_{c}^{T} S_{c^{\prime}} s_{c, \mu} .
$$

We bound the terms separately.

For the second term, since $c$ is an unit speed curve, we have that $\left\|s_{c^{\prime}}\right\|_{2}=1$ and

$$
\begin{aligned}
\left\|\left(A_{c}^{T} A_{c}\right)^{-1} A_{c}^{T} S_{c^{\prime}} s_{c, \mu}\right\|_{c} & \leq\left\|S_{c^{\prime}} s_{c, \mu}\right\|_{2} \leq\left\|S_{c, \mu}\right\|_{\infty} \\
& =\left\|A_{c}\left(A_{c}^{T} A_{c}\right)^{-1} A_{c}^{T} \sigma_{c}\right\|_{\infty} \\
& \leq\left\|A_{c}\left(A_{c}^{T} A_{c}\right)^{-1} A_{c}^{T} \sigma_{c}\right\|_{2} \\
& \leq\left\|\sigma_{c}\right\|_{2} \leq \sqrt{n} .
\end{aligned}
$$

For the first term,

$$
\begin{aligned}
\frac{d}{d t}\left(A_{c} \mu(c)\right)= & \frac{d}{d t}\left(P_{c} \operatorname{diag}\left(P_{c}\right)\right) \\
= & -S_{c^{\prime}} P_{c} \operatorname{diag}\left(P_{c}\right)-P_{c} S_{c^{\prime}} \operatorname{diag}\left(P_{c}\right)+2 P_{c} S_{c^{\prime}} P_{c} \operatorname{diag}\left(P_{c}\right) \\
& -P_{c} \operatorname{diag}\left(S_{c^{\prime}} P_{c}\right)-P_{c} \operatorname{diag}\left(P_{c} S_{c^{\prime}}\right)+2 P_{c} \operatorname{diag}\left(P_{c} S_{c^{\prime}} P_{c}\right)
\end{aligned}
$$

Using $\left\|s_{\mathcal{C}^{\prime}}\right\|_{2}=1$, we have

$$
\begin{aligned}
\left\|\frac{d}{d t} \mu(c)\right\|_{c} \leq & \left\|S_{c^{\prime}} A_{c} \mu(c)\right\|_{2} \\
& +\left\|S_{c^{\prime}} P_{c} \operatorname{diag}\left(P_{c}\right)\right\|_{2}+2\left\|P_{c} S_{c^{\prime}} \operatorname{diag}\left(P_{c}\right)\right\|_{2}+2\left\|P_{c} S_{c^{\prime}} P_{c} \operatorname{diag}\left(P_{c}\right)\right\|_{2} \\
& +\left\|P_{c} \operatorname{diag}\left(P_{c} S_{c^{\prime}}\right)\right\|_{2}+\left\|P_{c} \operatorname{diag}\left(P_{c} S_{c^{\prime}} P_{c}\right)\right\|_{2} \\
\leq & 8\left\|S_{c^{\prime}}\right\|_{\infty} \sqrt{n} \leq 8 \sqrt{n} .
\end{aligned}
$$

Combining both terms, we have the result.

\subsection{Smoothness of the metric $\left(G_{1}\right)$}

Lemma 58. Let $\gamma(t)$ be a geodesic on $M_{L}$ with $V(\gamma) \leq V_{0}$. Let $f(t)=\log \operatorname{det}\left(A_{\gamma(t)}^{T} A_{\gamma(t)}\right)$. Then, we have

$$
\sup _{0 \leq t \leq \ell}\left|f^{\prime \prime \prime}(t)\right|=O\left(\sqrt{h}+\sqrt{\frac{\log n}{n}}\right) .
$$

Hence, $G_{1}=O\left(\sqrt{\frac{\log n}{n}}+\sqrt{h}\right)$.

Proof. Note that

$$
f^{\prime}(t)=-2 \operatorname{Tr}\left(A_{\gamma}^{T} A_{\gamma}\right)^{-1} A_{\gamma}^{T} S_{\gamma^{\prime}} A_{\gamma}=-2 \operatorname{Tr} P_{\gamma} S_{\gamma^{\prime}}
$$


Since $\frac{d}{d t} P_{\gamma}=-S_{\gamma^{\prime}} P_{\gamma}-P_{\gamma} S_{\gamma^{\prime}}+2 P_{\gamma} S_{\gamma^{\prime}} P_{\gamma}$ and $\frac{d}{d s} S_{\gamma^{\prime}}=-S_{\gamma^{\prime}}^{2}+S_{\gamma^{\prime \prime}}$, we have

$$
\begin{aligned}
f^{\prime \prime}(t)= & 2 \operatorname{Tr} S_{\gamma^{\prime}} P_{\gamma} S_{\gamma^{\prime}}+2 \operatorname{Tr} P_{\gamma} S_{\gamma^{\prime}}^{2}-4 \operatorname{Tr} P_{\gamma} S_{\gamma^{\prime}} P_{\gamma} S_{\gamma^{\prime}} \\
& +2 \operatorname{Tr} P_{\gamma} S_{\gamma^{\prime}}^{2}-2 \operatorname{Tr} P_{\gamma} S_{\gamma^{\prime \prime}} \\
= & -4 \operatorname{Tr} P_{\gamma} S_{\gamma^{\prime}} P_{\gamma} S_{\gamma^{\prime}}+6 \operatorname{Tr} P_{\gamma} S_{\gamma^{\prime}}^{2}-2 \operatorname{Tr} P_{\gamma} S_{\gamma^{\prime \prime}}
\end{aligned}
$$

So, we have

$$
\begin{aligned}
f^{\prime \prime \prime}(t)= & +8 \operatorname{Tr} S_{\gamma^{\prime}} P_{\gamma} S_{\gamma^{\prime}} P_{\gamma} S_{\gamma^{\prime}}+8 \operatorname{Tr} P_{\gamma} S_{\gamma^{\prime}}^{2} P_{\gamma} S_{\gamma^{\prime}}-16 \operatorname{Tr} P_{\gamma} S_{\gamma^{\prime}} P_{\gamma} S_{\gamma^{\prime}} P_{\gamma} S_{\gamma^{\prime}} \\
& +8 \operatorname{Tr} P_{\gamma} S_{\gamma^{\prime}}^{2} P_{\gamma} S_{\gamma^{\prime}}-8 \operatorname{Tr} P_{\gamma} S_{\gamma^{\prime \prime}} P_{\gamma^{\prime}} S_{\gamma^{\prime}} \\
& -6 \operatorname{Tr} S_{\gamma^{\prime}} P_{\gamma^{\prime}} S_{\gamma^{\prime}}^{2}-6 \operatorname{Tr} P_{\gamma} S_{\gamma^{\prime}} S_{\gamma^{\prime}}^{2}+12 \operatorname{Tr} P_{\gamma} S_{\gamma^{\prime}} P_{\gamma} S_{\gamma^{\prime}}^{2} \\
& -12 \operatorname{Tr} P_{\gamma} S_{\gamma^{\prime}}^{3}+12 \operatorname{Tr} P_{\gamma} S_{\gamma^{\prime}} S_{\gamma^{\prime \prime}} \\
& +2 \operatorname{Tr} S_{\gamma^{\prime}} P_{\gamma^{\prime}} S_{\gamma^{\prime \prime}}+2 \operatorname{Tr} P_{\gamma} S_{\gamma^{\prime}} S_{\gamma^{\prime \prime}}-4 \operatorname{Tr} P_{\gamma} S_{\gamma^{\prime}} P_{\gamma} S_{\gamma^{\prime \prime}} \\
& +2 \operatorname{Tr} P_{\gamma} S_{\gamma^{\prime}} S_{\gamma^{\prime \prime}}-2 \operatorname{Tr} P_{\gamma} S_{\gamma^{\prime \prime \prime}} \\
= & -16 \operatorname{Tr} P_{\gamma} S_{\gamma^{\prime}} P_{\gamma} S_{\gamma^{\prime}} P_{\gamma} S_{\gamma^{\prime}}+36 \operatorname{Tr} P_{\gamma} S_{\gamma^{\prime}}^{2} P_{\gamma} S_{\gamma^{\prime}} \\
& -12 \operatorname{Tr} P_{\gamma} S_{\gamma^{\prime \prime}} P_{\gamma} S_{\gamma^{\prime}}-24 \operatorname{Tr} P_{\gamma} S_{\gamma^{\prime}}^{3} \\
& +16 \operatorname{Tr} P_{\gamma} S_{\gamma^{\prime}} S_{\gamma^{\prime \prime}}-2 \operatorname{Tr} P_{\gamma} \frac{d}{d t} S_{\gamma^{\prime \prime}} .
\end{aligned}
$$

Hence, we have

$$
\left|f^{\prime \prime \prime}(t)\right| \leq(16+36+24) \sqrt{\operatorname{Tr} S_{\gamma^{\prime}}^{4} \operatorname{Tr} S_{\gamma^{\prime}}^{2}}+(12+16) \sqrt{\operatorname{Tr} S_{\gamma^{\prime \prime}}^{2} \operatorname{Tr} S_{\gamma^{\prime}}^{2}}+2\left|\operatorname{Tr} P_{\gamma} \frac{d}{d t} S_{\gamma^{\prime \prime}}\right| .
$$

Since $\operatorname{Tr} S_{\gamma^{\prime}}^{2} \leq 48, \operatorname{Tr} S_{\gamma^{\prime}}^{4} \leq 48^{4} n^{-1}, \operatorname{Tr} S_{\gamma^{\prime \prime}}^{2} \leq 10^{8} n^{-1}$ (Lemma 54), we have

$$
\left|f^{\prime \prime \prime}(t)\right|=O\left(n^{-1 / 2}\right)+2\left|\operatorname{Tr} P_{\gamma} \frac{d}{d t} S_{\gamma^{\prime \prime}}\right|
$$

To bound the last term, we start with the geodesic equation:

$$
s_{\gamma^{\prime \prime}}=P_{\gamma} s_{\gamma^{\prime}}^{2}
$$

Since $\frac{d}{d t} P_{\gamma}=-S_{\gamma^{\prime}} P_{\gamma}-P_{\gamma} S_{\gamma^{\prime}}+2 P_{\gamma} S_{\gamma^{\prime}} P_{\gamma}$, we have that

$$
\begin{aligned}
\frac{d}{d t} s_{\gamma^{\prime \prime}}= & -S_{\gamma^{\prime}} P_{\gamma^{\prime}} s_{\gamma^{\prime}}^{2}-P_{\gamma} S_{\gamma^{\prime}} s_{\gamma^{\prime}}^{2}+2 P_{\gamma} S_{\gamma^{\prime}} P_{\gamma} s_{\gamma^{\prime}}^{2} \\
& +2 P_{\gamma} s_{\gamma^{\prime}} s_{\gamma^{\prime \prime}}-2 P_{\gamma} s_{\gamma^{\prime}}^{3} \\
= & -S_{\gamma^{\prime}} P_{\gamma} s_{\gamma^{\prime}}^{2}-3 P_{\gamma} s_{\gamma^{\prime}}^{3}+2 P_{\gamma} S_{\gamma^{\prime}} P_{\gamma} s_{\gamma^{\prime}}^{2}+2 P_{\gamma} s_{\gamma^{\prime}} s_{\gamma^{\prime \prime}} \\
= & -S_{\gamma^{\prime}} P_{\gamma} s_{\gamma^{\prime}}^{2}-3 P_{\gamma} s_{\gamma^{\prime}}^{3}+4 P_{\gamma} S_{\gamma^{\prime}} P_{\gamma} s_{\gamma^{\prime}}^{2} .
\end{aligned}
$$


Hence, we have that

$$
\begin{aligned}
\left|\operatorname{Tr} P_{\gamma} \frac{d}{d t} S_{\gamma^{\prime \prime}}\right| & \leq\left|\sigma_{\gamma}^{T} S_{\gamma^{\prime}} P_{\gamma} s_{\gamma^{\prime}}^{2}\right|+3\left|\sigma_{\gamma}^{T} P_{\gamma} s_{\gamma^{\prime}}^{3}\right|+4\left|\sigma_{\gamma}^{T} P_{\gamma} S_{\gamma^{\prime}} P_{\gamma^{\prime}} s_{\gamma^{\prime}}^{2}\right| \\
& =\left|\left(S_{\gamma^{\prime}} \sigma_{\gamma}\right)^{T} P_{\gamma^{\prime}} s_{\gamma^{\prime}}^{2}\right|+3\left|\left(S_{\gamma^{\prime}} P_{\gamma} \sigma_{\gamma}\right)^{T} s_{\gamma^{\prime}}^{2}\right|+4\left(S_{\gamma^{\prime}} P_{\gamma} \sigma_{\gamma}\right)^{T}\left(P_{\gamma} s_{\gamma^{\prime}}^{2}\right) \\
& \leq \sqrt{\sum s_{\gamma^{\prime}}^{2} \sum s_{\gamma^{\prime}}^{4}}+\sqrt{\sum s_{\gamma^{\prime}}^{4}} \sqrt{\sigma_{\gamma}^{T} P_{\gamma} S_{\gamma^{\prime}}^{2} P_{\gamma} \sigma_{\gamma}} \\
& =O\left(n^{-1 / 2}\right)+O\left(n^{-1}\right) O\left(\sqrt{\frac{\log n}{n}}+\sqrt{h}\right) O(n) \\
& \leq O\left(\sqrt{\frac{\log n}{n}}+\sqrt{h}\right) .
\end{aligned}
$$

Hence, we have that

$$
\left|f^{\prime \prime \prime}(t)\right| \leq O\left(\sqrt{\frac{\log n}{n}}+\sqrt{h}\right) .
$$

\subsection{Stability of Curvatures $\left(R_{1}\right.$ and $\left.R_{2}\right)$}

Lemma 59. Let $\gamma(t)$ be a geodesic on $M_{L}$ with $V(\gamma) \leq V_{0}$. Then, we have that

$$
\sup _{0 \leq t \leq \ell}\|R(t)\|_{F}=O\left(n^{-1 / 2}\right)
$$

where $R(t)$ defined in Definition 8. Hence, $R_{1}=O\left(n^{-1 / 2}\right)$.

Proof. Let $\overline{R(t)}$ such that $\left\langle R\left(u, \gamma^{\prime}(t)\right) \gamma^{\prime}(t), v\right\rangle=u^{T} \overline{R(t)} v$. Lemma 48 shows that

$$
u^{T} \overline{R(t)} v=s_{u}^{T} S_{\gamma^{\prime}} P_{\gamma} S_{\gamma^{\prime}} s_{v}-\left(s_{v} s_{u}\right)^{T} P_{\gamma} s_{\gamma^{\prime}}^{2}
$$

Hence, we have

$$
\overline{R(t)}=A_{\gamma}^{T} S_{\gamma^{\prime}} P_{\gamma} S_{\gamma^{\prime}} A_{\gamma}-A_{\gamma}^{T} \operatorname{Diag}\left(P_{\gamma} s_{\gamma^{\prime}}^{2}\right) A_{\gamma}
$$

Pick $X_{i}=\left(A^{T} A_{\gamma}\right)^{-1 / 2} e_{i}$. Then, we can write $R(t)$ in the coordinate systems $\left\{X_{i}\right\}_{i}$ and get

$$
R(t)=\left(A_{\gamma}^{T} A_{\gamma}\right)^{-1 / 2}\left(A_{\gamma}^{T} S_{\gamma^{\prime}} P_{\gamma} S_{\gamma^{\prime}} A_{\gamma}-A_{\gamma}^{T} \operatorname{Diag}\left(P_{\gamma} s_{\gamma^{\prime}}^{2}\right) A_{\gamma}\right)\left(A_{\gamma}^{T} A_{\gamma}\right)^{-1 / 2} .
$$

Therefore, we have that

$$
\begin{aligned}
& \|R(t)\|_{F}^{2} \\
\leq & 2\left\|\left(A_{\gamma}^{T} A_{\gamma}\right)^{-1 / 2} A_{\gamma}^{T} S_{\gamma^{\prime}} P_{\gamma} S_{\gamma^{\prime}} A_{\gamma}\left(A_{\gamma}^{T} A_{\gamma}\right)^{-1 / 2}\right\|_{F}^{2}+2\left\|\left(A_{\gamma}^{T} A_{\gamma}\right)^{-1 / 2} A_{\gamma}^{T} \operatorname{Diag}\left(P_{\gamma} s_{\gamma^{\prime}}^{2}\right) A_{\gamma}\left(A_{\gamma}^{T} A_{\gamma}\right)^{-1 / 2}\right\|_{F}^{2} \\
= & 2 \operatorname{Tr} P_{\gamma} S_{\gamma^{\prime}} P_{\gamma} S_{\gamma^{\prime}} P_{\gamma} S_{\gamma^{\prime}} P_{\gamma} S_{\gamma^{\prime}}+2 \operatorname{Tr} P_{\gamma} \operatorname{Diag}\left(P_{\gamma} s_{\gamma^{\prime}}^{2}\right) P_{\gamma} \operatorname{Diag}\left(P_{\gamma} s_{\gamma^{\prime}}^{2}\right) \\
\leq & 4\left\|s_{\gamma^{\prime}}\right\|_{4}^{4} .
\end{aligned}
$$

The claim follows from Lemma 54 . 
Lemma 60. Given a geodesic $\gamma(t)$ on $M_{L}$ with $V(\gamma) \leq V_{0}$. Assume that $h \leq \frac{1}{\sqrt{n}}$. For any $t$ such that $0 \leq t \leq \ell$, any curve $c(s)$ starting from $\gamma(t)$ and any vector field $v(s)$ on $c(s)$ with $v(0)=\gamma^{\prime}(t)$, we have that

$$
\left|\frac{d}{d s} \operatorname{Ric}(v(s))\right|_{s=0} \mid \leq O\left(1+\sqrt{\frac{\log n}{n h}}\right)\left(\left\|\frac{d c}{d s}\right\|+\ell\left\|D_{s} v\right\|\right) .
$$

Therefore, $R_{2}=O\left(1+\sqrt{\frac{\log n}{n h}}\right)$.

Proof. By Lemma 48, we know that

$$
\begin{aligned}
\operatorname{Ric}(v(s)) & =s_{c(s), v(s)}^{T} P_{c(s)}^{(2)} s_{c(s), v(s)}-\sigma_{c(s)}^{T} P_{c(s)} s_{c(s), v(s)}^{2} \\
& =\operatorname{Tr}\left(S_{c(s), v(s)} P_{c(s)} S_{c(s), v(s)} P_{c(s)}\right)-\operatorname{Tr}\left(\operatorname{Diag}\left(P_{c(s)} s_{c(s), v(s)}^{2}\right) P_{c(s)}\right) .
\end{aligned}
$$

Note that the "s" in $c(s)$ is the parameter of the curve $s$ while the first letter "s" in both $s_{c(s)}$ and $S_{c(s)}$ denotes the slack. For simplicity, we suppress the parameter $s$ and hence, we have

$$
\operatorname{Ric}(v)=\operatorname{Tr}\left(S_{c, v} P_{c} S_{c, v} P_{c}\right)-\operatorname{Tr}\left(\operatorname{Diag}\left(P_{c} S_{c, v}^{2}\right) P_{c}\right)
$$

We write $\frac{d}{d s} c=c_{s}$ and $\frac{d}{d s} v=v_{s}$ (in Euclidean coordinate). Since $\frac{d}{d s} P_{c}=-S_{c_{s}} P_{c}-P_{c} S_{c_{s}}+2 P_{c} S_{c_{s}} P_{c}$ and $\frac{d}{d s} S_{c, v}=-S_{c_{s}} S_{c, v}+S_{c, v_{s}}$, we have that

$$
\begin{aligned}
& \frac{d}{d s} \operatorname{Ric}(v) \\
= & -2 \operatorname{Tr}\left(S_{c, v} S_{c_{s}} P_{c} S_{c, v} P_{c}\right)-2 \operatorname{Tr}\left(S_{c, v} P_{c} S_{c_{s}} S_{c, v} P_{c}\right)+4 \operatorname{Tr}\left(S_{c, v} P_{c} S_{c_{s}} P_{c} S_{c, v} P_{c}\right) \\
& -2 \operatorname{Tr}\left(S_{c_{s}} S_{c, v} P_{c} S_{c, v} P_{c}\right)+2 \operatorname{Tr}\left(S_{c, v_{s}} P_{c} S_{c, v} P_{c}\right) \\
& +\operatorname{Tr}\left(\operatorname{Diag}\left(P_{c} s_{c, v}^{2}\right) S_{c_{s}} P_{c}\right)+\operatorname{Tr}\left(\operatorname{Diag}\left(P_{c} s_{c, v}^{2}\right) P_{c} S_{c_{s}}\right)-2 \operatorname{Tr}\left(\operatorname{Diag}\left(P_{c} s_{c, v}^{2}\right) P_{c} S_{c_{s}} P_{c}\right) \\
& +\operatorname{Tr}\left(\operatorname{Diag}\left(P_{c} S_{c_{s}} s_{c, v}^{2}\right) P_{c}\right)+\operatorname{Tr}\left(\operatorname{Diag}\left(S_{c_{s}} P_{c} s_{c, v}^{2}\right) P_{c}\right)-2 \operatorname{Tr}\left(\operatorname{Diag}\left(P_{c} S_{c_{s}} P_{c} s_{c, v}^{2}\right) P_{c}\right) \\
& +2 \operatorname{Tr}\left(\operatorname{Diag}\left(P_{c} S_{c, v} S_{c_{s}} s_{c, v}\right) P_{c}\right)-2 \operatorname{Tr}\left(\operatorname{Diag}\left(P_{c} S_{c, v} s_{c, v_{s}}\right) P_{c}\right) \\
= & -6 \operatorname{Tr}\left(S_{c, v} S_{c_{s}} P_{c} S_{c, v} P_{c}\right)+4 \operatorname{Tr}\left(S_{c, v} P_{c} S_{c_{s}} P_{c} S_{c, v} P_{c}\right)+2 \operatorname{Tr}\left(S_{c, v_{s}} P_{c} S_{c, v} P_{c}\right) \\
& +3 \operatorname{Tr}\left(\operatorname{Diag}\left(P_{c} s_{c, v}^{2}\right) S_{c_{s}} P_{c}\right)-2 \operatorname{Tr}\left(\operatorname{Diag}\left(P_{c} s_{c, v}^{2}\right) P_{c} S_{c_{s}} P_{c}\right) \\
& +3 \operatorname{Tr}\left(\operatorname{Diag}\left(P_{c} S_{c_{s}} s_{c, v}^{2}\right) P_{c}\right)-2 \operatorname{Tr}\left(\operatorname{Diag}\left(P_{c} S_{c_{s}} P_{c} s_{c, v}^{2}\right) P_{c}\right) \\
& -2 \operatorname{Tr}\left(\operatorname{Diag}\left(P_{c} S_{c, v} s_{c, v_{s}}\right) P_{c}\right) .
\end{aligned}
$$

Let $\frac{d}{d s} \operatorname{Ric}(v)=(1)+(2)$ where (1) is the sum of all terms not involving $v_{s}$ and (2) is the sum of other terms. 
For the first term (1), we have that

$$
\begin{aligned}
|(1)| \leq & 6\left|\operatorname{Tr}\left(S_{c, v} S_{c_{s}} P_{c} S_{c, v} P_{c}\right)\right|+4\left|\operatorname{Tr}\left(S_{c, v} P_{c} S_{c_{s}} P_{c} S_{c, v} P_{c}\right)\right| \\
& +3\left|\operatorname{Tr}\left(\operatorname{Diag}\left(P_{c} s_{c, v}^{2}\right) S_{c_{s}} P_{c}\right)\right|+2\left|\operatorname{Tr}\left(\operatorname{Diag}\left(P_{c} s_{c, v}^{2}\right) P_{c} S_{c_{s}} P_{c}\right)\right| \\
& +3\left|\operatorname{Tr}\left(\operatorname{Diag}\left(P_{c} S_{c_{s}} s_{c, v}^{2}\right) P_{c}\right)\right|+2\left|\operatorname{Tr}\left(\operatorname{Diag}\left(P_{c} S_{c_{s}} P_{c} s_{c, v}^{2}\right) P_{c}\right)\right| \\
\leq & 6\left\|S_{c_{s}}\right\|_{\infty} \sqrt{\sum_{i}\left(s_{c, v}\right)_{i}^{2}} \sqrt{\sum_{i}\left(s_{c, v}\right)_{i}^{2}}+4\left\|S_{c_{s}}\right\|_{\infty}\left|\operatorname{Tr}\left(P_{c} S_{c, v} P_{c} S_{c, v} P_{c}\right)\right| \\
& +3 \sqrt{\sum_{i}\left(s_{c, v}\right)_{i}^{4}}\left\|S_{c_{s}}\right\|_{2}+2 \sqrt{\sum_{i}\left(s_{c, v}\right)_{i}^{4}} \sqrt{\sum_{i}\left(P_{c} S_{c_{s}} P_{c}\right)_{i i}^{2}} \\
& +3\left\|S_{c_{s}}\right\|_{\infty} \sqrt{\sum_{i}\left(s_{c, v}\right)_{i}^{4}} \sqrt{\sum_{i}\left(P_{c}\right)_{i i}^{2}}+2\left\|S_{c_{s}}\right\|_{\infty} \sqrt{\sum_{i}\left(s_{c, v}\right)_{i}^{4}} \sqrt{\sum_{i}\left(P_{c}\right)_{i i}^{2}} \\
\leq & 10\left\|S_{c_{s}}\right\|_{\infty}\left\|S_{c, v}\right\|_{2}^{2}+3\left\|S_{c, v}\right\|_{4}^{2}\left\|S_{c_{s}}\right\|_{2}+7\left\|S_{c_{s}}\right\|_{\infty}\left\|S_{c, v}\right\|_{4}^{2} \sqrt{n} \\
\leq & 20\left\|S_{c_{s}}\right\|_{2}\left\|S_{c, v}\right\|_{4}^{2} \sqrt{n} .
\end{aligned}
$$

Since $s_{c, v}=s_{\gamma^{\prime}}$ at $s=0$, we have that $\left\|S_{c, v}\right\|_{4}^{2} \leq 48^{2} n^{-1 / 2}$ and hence

$$
|(1)|=O(1)\left\|s_{c_{s}}\right\|_{2} \text {. }
$$

For the second term (2), we have that

$$
\begin{aligned}
|(2)| & \leq 2\left|\operatorname{Tr}\left(S_{c, v_{s}} P_{c} S_{c, v} P_{c}\right)\right|+2\left|\operatorname{Tr}\left(\operatorname{Diag}\left(P_{c} S_{c, v} s_{c, v_{s}}\right) P_{c}\right)\right| \\
& \leq 2\left\|s_{c, v_{s}}\right\|_{2}\left\|s_{c, v}\right\|_{2}+2 \sqrt{n} \sqrt{\sum_{i}\left(s_{c, v_{s}} s_{c, v}\right)_{i}^{2}} \\
& \leq O(1)\left\|s_{c, v_{s}}\right\|_{2}+O(\sqrt{\log n}+\sqrt{n h})\left\|s_{c, v_{s}}\right\|_{2} \\
& =O(\sqrt{\log n}+\sqrt{n h})\left\|s_{c, v_{s}}\right\|_{2}
\end{aligned}
$$

where we used $\left\|s_{c, v}\right\|_{\infty}=\left\|s_{\gamma^{\prime}}\right\|_{\infty}=O\left(\sqrt{\frac{\log n}{n}}+\sqrt{h}\right)$ and $\left\|s_{c, v}\right\|_{2}=\left\|s_{\gamma^{\prime}}\right\|_{2}=O(1)$ at $s=0$ in the second last line.

Note that at $s=0$, we have

$$
D_{s} v=\frac{d v}{d s}-\left(A_{c}^{T} A_{c}\right)^{-1} A_{c}^{T} S_{c_{s}} s_{c, v} .
$$

Therefore, we have

$$
s_{c, v_{s}}=A_{c}\left(D_{s} v\right)-A_{c}\left(A_{c}^{T} A_{c}\right)^{-1} A_{c}^{T} S_{c_{s}} s_{c, v}
$$

and hence

$$
\begin{aligned}
\left\|s_{c, v_{s}}\right\|_{2} & \leq\left\|D_{s} v\right\|+\left\|A_{c}\left(A_{c}^{T} A_{c}\right)^{-1} A_{c}^{T} S_{c_{s}} s_{c, v}\right\|_{2} \\
& \leq\left\|D_{s} v\right\|+\left\|s_{\gamma^{\prime}}\right\|_{\infty}\left\|s_{c_{s}}\right\|_{2}
\end{aligned}
$$

Therefore, we have

$$
|(2)|=O(\sqrt{\log n}+\sqrt{n h})\left(\left\|D_{s} v\right\|+\left(\sqrt{\frac{\log n}{n}}+\sqrt{h}\right)\left\|s_{c_{s}}\right\|_{2}\right) .
$$


Therefore, we have

$$
\begin{aligned}
\left|\frac{d}{d s} \operatorname{Ric}(v(s))\right|_{s=0} \mid= & O(1)\left\|s_{c_{s}}\right\|_{2}+O(\sqrt{\log n}+\sqrt{n h})\left\|D_{s} v\right\| \\
& +O(\sqrt{\log n}+\sqrt{n h})\left(\sqrt{\frac{\log n}{n}}+\sqrt{h}\right)\left\|s_{c_{s}}\right\|_{2} \\
= & O\left(1+\sqrt{\frac{\log n}{n h}}\right)\left(\left\|\frac{d c}{d s}\right\|+\ell\left\|D_{s} v\right\|\right) .
\end{aligned}
$$

where we used $h \leq \frac{1}{\sqrt{n}}$ at the last line.

\subsection{Stability of $L_{4}+L_{\infty}$ norm $\left(V_{1}\right)$}

Lemma 61. Given a family of geodesic $\gamma_{s}(t)$ on $M_{L}$ with $V\left(\gamma_{0}\right) \leq V_{0}$. Suppose that $h \leq \frac{1}{\sqrt{n}}$, we have that

$$
\left|\frac{d}{d s} V\left(\gamma_{s}\right)\right| \leq O\left(\frac{1}{\sqrt{n} h+\sqrt{h \log n}}\right)\left(\left\|\frac{d}{d s} \gamma_{s}(0)\right\|_{\gamma_{s}(0)}+\ell\left\|D_{s} \gamma_{s}^{\prime}\right\|_{\gamma_{s}(0)}\right) .
$$

Hence, we have that $V_{1}=O\left(\frac{1}{\sqrt{n} h+\sqrt{h \log n}}\right)$.

Proof. Since $\frac{d}{d s} s_{\gamma^{\prime}}=-s_{\gamma^{\prime}} A_{\gamma} \frac{d}{d s} \gamma+A_{\gamma} \frac{d}{d s} \gamma^{\prime}$, we have

$$
\left\|\frac{d}{d s} s_{\gamma^{\prime}}\right\|_{2} \leq\left\|s_{\gamma^{\prime}}\right\|_{\infty}\left\|A_{\gamma} \frac{d}{d s} \gamma\right\|_{2}+\left\|A_{\gamma} \frac{d}{d s} \gamma^{\prime}\right\|_{2} .
$$

For the last term, we note that

$$
D_{s} \gamma^{\prime}=\frac{d \gamma^{\prime}}{d s}-\left(A_{\gamma}^{T} A_{\gamma}\right)^{-1} A_{\gamma}^{T} S_{\frac{d \gamma}{d s}} s_{\gamma^{\prime}}
$$

Hence, we have

$$
\left\|A_{\gamma} \frac{d}{d s} \gamma^{\prime}\right\|_{2} \leq\left\|D_{s} \gamma^{\prime}\right\|+\left\|S_{\frac{d \gamma}{d s}} s_{\gamma^{\prime}}\right\|_{2} \leq\left\|D_{s} \gamma^{\prime}\right\|+\left\|s_{\gamma^{\prime}}\right\|_{\infty}\left\|S_{\frac{d \gamma}{d s}}\right\|_{2} .
$$

Therefore, we have that

$$
\begin{aligned}
\left\|\frac{d}{d s} s_{\gamma^{\prime}}\right\|_{2} & \leq 2\left\|s_{\gamma^{\prime}}\right\|_{\infty}\left\|A_{\gamma} \frac{d}{d s} \gamma\right\|_{2}+\left\|D_{s} \gamma^{\prime}\right\| \\
& =O\left(\sqrt{\frac{\log n}{n}}+\sqrt{h}\right)\left\|\frac{d}{d s} \gamma\right\|+\left\|D_{s} \gamma^{\prime}\right\|
\end{aligned}
$$

By Lemma 59, we have that $\|R(t)\|_{F}=O\left(n^{-1 / 2}\right)$. Since $\gamma_{s}$ is a family of geodesic, $\frac{d}{d s} \gamma(t)$ is a Jacobi field and Lemma 41 shows that

$$
\left\|\frac{d}{d s} \gamma(t)-\alpha-\beta t\right\| \leq \lambda t^{2}\left\|\frac{d}{d s} \gamma_{s}(0)\right\|+\frac{\lambda t^{3}}{5}\left\|D_{s} \gamma_{s}^{\prime}(0)\right\|
$$

and

$$
\left\|D_{t} \frac{d}{d s} \gamma(t)-\beta\right\| \leq 2 \lambda t\left\|\frac{d}{d s} \gamma_{s}(0)\right\|+\frac{3 \lambda t^{2}}{5}\left\|D_{s} \gamma_{s}^{\prime}(0)\right\|
$$


with $\lambda=O\left(n^{-1 / 2}\right)$. Using $h \leq \frac{1}{\sqrt{n}}$, for any $0 \leq t \leq \ell$, we have that

$$
\left\|\frac{d}{d s} \gamma(t)\right\| \leq O(1)\left(\left\|\frac{d}{d s} \gamma_{s}(0)\right\|+\ell\left\|D_{s} \gamma_{s}^{\prime}(0)\right\|\right)
$$

and

$$
\ell\left\|D_{t} \frac{d}{d s} \gamma(t)\right\| \leq O(1)\left(\left\|\frac{d}{d s} \gamma_{s}(0)\right\|+\ell\left\|D_{s} \gamma_{s}^{\prime}(0)\right\|\right) .
$$

Putting these into (4.7) and using $h \leq \frac{1}{\sqrt{n}}$, we have

$$
\begin{aligned}
\left\|\frac{d}{d s} s_{\gamma^{\prime}}\right\|_{2} & =O\left(\sqrt{\frac{\log n}{n}}+\sqrt{h}+\frac{1}{\sqrt{n h}}\right)\left(\left\|\frac{d}{d s} \gamma_{s}(0)\right\|+\ell\left\|D_{s} \gamma_{s}^{\prime}(0)\right\|\right) \\
& =O\left(\frac{1}{\sqrt{n h}}\right)\left(\left\|\frac{d}{d s} \gamma_{s}(0)\right\|+\ell\left\|D_{s} \gamma_{s}^{\prime}(0)\right\|\right) .
\end{aligned}
$$

Hence, we have that

$$
\begin{aligned}
\frac{d}{d s}\left(\frac{\left\|s_{\gamma^{\prime}(t)}\right\|_{4}}{n^{-1 / 4}}+\frac{\left\|s_{\gamma^{\prime}(t)}\right\|_{\infty}}{\sqrt{\frac{\log n}{n}}+\sqrt{h}}\right) & \leq \frac{\left\|\frac{d}{d s} s_{\gamma^{\prime}(t)}\right\|_{4}}{n^{-1 / 4}}+\frac{\left\|\frac{d}{d s} s_{\gamma^{\prime}(t)}\right\|_{\infty}}{\sqrt{\frac{\log n}{n}}+\sqrt{h}} \\
& =O\left(\frac{1}{\sqrt{\frac{\log n}{n}}+\sqrt{h}}\right)\left\|\frac{d}{d s} s_{\gamma^{\prime}(t)}\right\|_{2} \\
& =O\left(\frac{1}{\sqrt{n} h+\sqrt{h \log n}}\right)\left(\left\|\frac{d}{d s} \gamma_{s}(0)\right\|+\ell\left\|D_{s} \gamma_{s}^{\prime}(0)\right\|\right) .
\end{aligned}
$$

Hence, we have that the result.

\subsection{Mixing Time}

Proof of Theorem 45. In the last previous sections, we proved that if $h \leq \frac{1}{1000 \sqrt{n}}$

1. $V_{0}=48(4.6)$

2. $V_{1}=O\left(\frac{1}{\sqrt{n} h+\sqrt{h \log n}}\right)($ Lemma 61$)$

3. $D_{0}=O(\sqrt{n})($ Lemma 55$)$

4. $D_{1}=O(n \sqrt{h}+\sqrt{n \log n})($ Lemma 56)

5. $D_{2}=O(\sqrt{n})($ Lemma 57$)$

6. $G_{1}=O\left(\sqrt{h}+\sqrt{\frac{\log n}{n}}\right)($ Lemma 58)

7. $G_{2}=O(\sqrt{m})($ Lemma 50$)$

8. $R_{1}=O(1 / \sqrt{n})($ Lemma 59)

9. $R_{2}=O\left(1+\sqrt{\frac{\log n}{n h}}\right)($ Lemma 60$)$ 
Lemma 53 proved that

$$
P(V(\gamma) \leq 24) \geq 1-\frac{3}{n} .
$$

Therefore, if we set $h=\Omega\left(n^{-1}\right)$, we have that

$$
P(V(\gamma) \leq 24) \geq 1-\frac{V_{0}}{100 V_{1}}
$$

Hence, these are valid parameters for $M_{L}$ with $H=\frac{1}{1000 \sqrt{n}}$.

Theorem 23 implies that the walk has mixing time $O\left(G_{2}^{2} / h\right)$ as long as

$$
h \leq \Theta(1) \min \left\{\frac{1}{n^{1 / 3} D_{1}^{2 / 3}}, \frac{1}{D_{2}}, \frac{1}{n R_{1}}, \frac{1}{\left(n D_{0} R_{1}\right)^{2 / 3}}, \frac{1}{\left(n R_{2}\right)^{2 / 3}}, \frac{1}{n G_{1}^{2 / 3}}, H\right\} \leq \frac{1}{\Theta\left(n^{3 / 4}\right)}
$$

if $n$ is large enough.

Remark. The bottleneck of the proof occurs in the parameters $D_{1}$ and $G_{1}$. 


\section{Collocation Method for ODE}

In this section, we study a collocation method for solving ordinary differential equation (ODE) and show how to solve a nice enough ODE in nearly constant number of iterations without explicitly computing higher derivatives.

Collocation method is a general framework for solving differential equations. This framework finds a solution to the differential equation by finding a low degree polynomial (or other finite dimensional space that approximate the function space) that satisfies the differential equation at a set of predesignated points (called collocation points). By choosing the finite dimensional space and the collocation points carefully, one can make sure there is an unique solution and that the solution can be found using simple iterative methods (See [12, Sec 3.4] for an introduction). One key departure of our analysis to the standard analysis is that we use $L_{4}$ norm (instead of the standard $L_{2}$ norm). This is essential to take advantage of the stability of the $L_{4}$ norm of the geodesic.

\subsection{First Order ODE}

We first consider the following first order ODE

$$
\begin{aligned}
\frac{d}{d t} u(t) & =F(u(t), t), \text { for } 0 \leq t \leq \ell \\
u(0) & =v
\end{aligned}
$$

where $F: \mathbb{R}^{n+1} \rightarrow \mathbb{R}^{n}$ and $u(t) \in \mathbb{R}^{n}$. The idea of collocation methods is to find a degree $d$ polynomial $p$ such that

$$
\begin{aligned}
\frac{d}{d t} p(t) & =F(p(t), t), \text { for } t=c_{1}, c_{2}, \cdots, c_{d} \\
p(0) & =v
\end{aligned}
$$

where $c_{1}, c_{2}, \cdots, c_{d}$ are carefully chosen distinct points on $[0, \ell]$. Here, we call $p: \mathbb{R} \rightarrow \mathbb{R}^{n}$ is a degree $d$ polynomial if $p(t)=\left[p_{1}(t) ; p_{2}(t) ; \cdots ; p_{n}(t)\right]$ and each $p_{i}(t)$ is an univariate polynomial with degree at most $d$. The first part of the proof shows the existence of a solution for the systems (5.2). To describe the algorithm, it is easier to consider an equivalent integral equation.

Lemma 62. Given distinct points $c_{1}, c_{2}, \cdots, c_{d} \in \mathbb{R}$ and $F: \mathbb{R}^{n+1} \rightarrow \mathbb{R}^{n}$, consider the nonlinear $\operatorname{map} T: \mathbb{R}^{d \times n} \rightarrow \mathbb{R}^{d \times n}$ defined by

$$
T(\zeta)_{(i, k)}=\int_{0}^{c_{i}} \sum_{j=1}^{d} F\left(\zeta_{j}, c_{j}\right)_{k} \phi_{j}(s) d s \text { for } i \in[d], k \in[n]
$$

where $\phi_{i}(s)=\prod_{j \neq i} \frac{s-c_{j}}{c_{i}-c_{j}}$ are the Lagrange basis polynomials. Given any $\zeta \in \mathbb{R}^{d \times n}$ such that

$$
\zeta_{i}=v+T(\zeta)_{i}, \text { for } i \in[d]
$$

the polynomial

$$
p(t)=v+\int_{0}^{t} \sum_{j=1}^{d} F\left(\zeta_{j}, c_{j}\right) \phi_{j}(s) d s
$$

is a solution of the system (5.2). 
Proof. Define the polynomials $\phi_{i}(s)=\prod_{j \neq i} \frac{s-c_{j}}{c_{i}-c_{j}}$. Note that $\phi_{i}\left(c_{j}\right)=\delta_{i j}$. Therefore, we have

$$
\sum_{j=1}^{d} \alpha_{j} \phi_{j}\left(c_{i}\right)=\alpha_{i}
$$

Therefore, $p(0)=v$ and

$$
\frac{d}{d t} p\left(c_{i}\right)=\sum_{j=1}^{d} F\left(\zeta_{j}, c_{j}\right) \phi_{j}\left(c_{i}\right)=F\left(\zeta_{j}, c_{j}\right) .
$$

Since $\zeta_{i}=v+T(\zeta)_{i}$, we have that

$$
\zeta_{i}=v+\int_{0}^{c_{i}} \sum_{j=1}^{d} F\left(\zeta_{j}, c_{j}\right) \phi_{j}(s) d s=p\left(c_{i}\right)
$$

Hence, we have $\frac{d}{d t} p\left(c_{i}\right)=F\left(p\left(c_{i}\right), c_{i}\right)$. Therefore, $p$ is a solution to the system (5.2).

From Lemma 62, we see that it suffices to solve the system (5.3). We solve it by a simple fix point iteration shown in Algorithm 3.

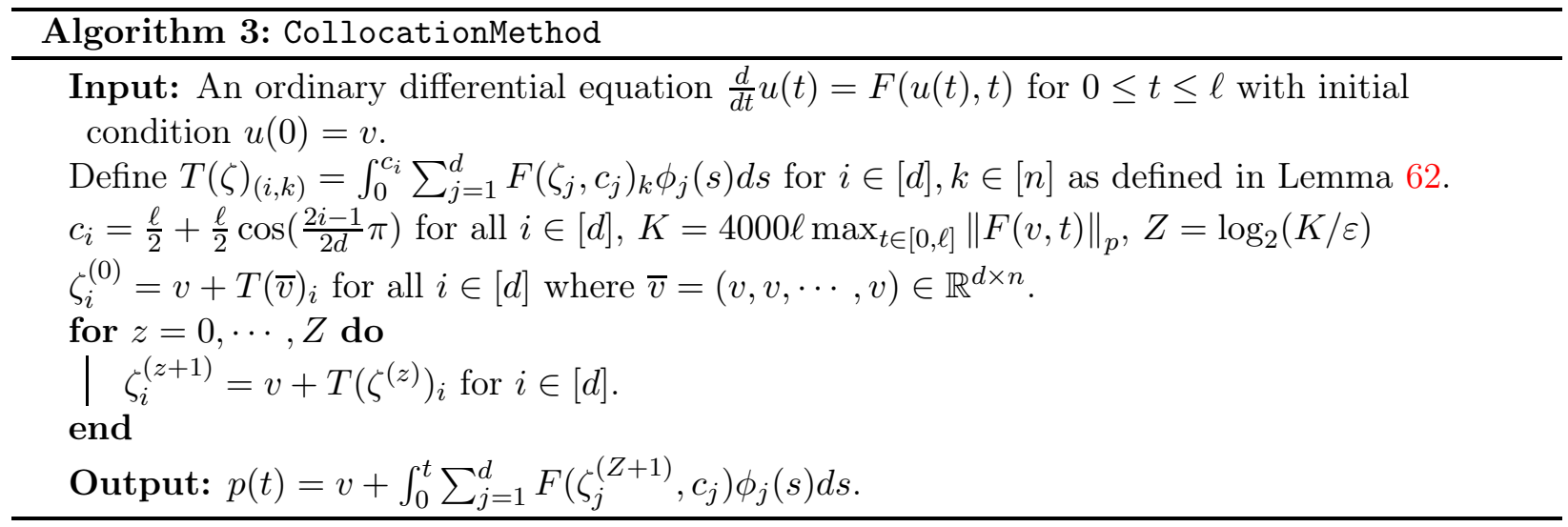

In the following lemma, we show that $T$ is a contraction mapping if $F$ is smooth enough and hence this algorithm converges linearly. We will use the following norm: $\|x\|_{\infty ; p}=\max _{i \in[d]}\left(\sum_{k \in[n]}\left|x_{(i, k)}\right|^{p}\right)^{1 / p}$.

Lemma 63. Given $x, y \in \mathbb{R}^{d \times n}$, suppose that $\left\|F\left(x_{i}, t\right)-F\left(y_{i}, t\right)\right\|_{p} \leq L\left\|x_{i}-y_{i}\right\|_{p}$ for all $0 \leq t \leq \ell$ and all $i \in[d]$. For $c_{k}=\frac{\ell}{2}+\frac{\ell}{2} \cos \left(\frac{2 k-1}{2 d} \pi\right)$ and $T$ defined Lemma 62, we have that

$$
\|T(x)-T(y)\|_{\infty ; p} \leq 1000 \ell L\|x-y\|_{\infty ; p} .
$$

Also, for any $\alpha \in \mathbb{R}^{d \times n}$, we have that

$$
\int_{0}^{t} \sum_{j=1}^{d} \alpha_{j} \phi_{j}(s) d s \leq 1000 \ell \max _{i}\left\|\alpha_{i}\right\|_{p}
$$


Proof. Using the definition of $T$ and $\|\cdot\|_{\infty ; p}$, we have

$$
\begin{aligned}
\|T(x)-T(y)\|_{\infty ; p} & =\max _{i}\left\|\int_{0}^{c_{i}} \sum_{j=1}^{d} F\left(x_{j}, c_{j}\right) \phi_{j}(s) d s-\int_{0}^{c_{i}} \sum_{j=1}^{d} F\left(y_{j}, c_{j}\right) \phi_{j}(s) d s\right\|_{p} \\
& \leq\left(\max _{i} \sum_{j=1}^{d}\left|\int_{0}^{c_{i}} \phi_{j}(s) d s\right|\right)\left(\max _{i}\left\|F\left(x_{i}, c_{i}\right)-F\left(y_{i}, c_{i}\right)\right\|_{p}\right) \\
& \leq L\|x-y\|_{\infty ; p}\left(\max _{i} \sum_{j=1}^{d}\left|\int_{0}^{c_{i}} \phi_{j}(s) d s\right|\right) .
\end{aligned}
$$

To bound $\max _{i} \sum_{j=1}^{d}\left|\int_{0}^{c_{i}} \phi_{j}(s) d s\right|$, it is easier to work with the function $\psi_{j}(x) \stackrel{\text { def }}{=} \phi_{j}\left(\frac{2}{\ell} x-1\right)$. Note that $\psi_{k}$ is the Lagrange basis polynomials on the nodes $\left\{\cos \left(\frac{2 k-1}{2 d} \pi\right)\right\}_{k=1}^{d}$ and hence we have

$$
\psi_{k}(x)=\frac{(-1)^{k-1} \sqrt{1-x_{k}^{2}} \cos \left(d \cos ^{-1} x\right)}{d\left(x-x_{k}\right)}
$$

where $x_{k}=\cos \left(\frac{2 k-1}{2 d} \pi\right)$. Lemma 91 at the end of this section shows that $\left|\int_{-1}^{t} \psi_{k}(x) d x\right| \leq \frac{2000}{d}$ for all $t$. Therefore, we have that $\max _{i} \sum_{j=1}^{d}\left|\int_{0}^{c_{i}} \phi_{j}(s) d s\right| \leq 1000 \ell$. This gives the first inequality. The second inequality is similar.

In each iteration of the collation method, we need to compute $\int_{0}^{c_{i}} \sum \alpha_{j} \phi_{j}(s) d s$ for some $\alpha_{j}$. The following theorem shows that this can be done in $O(d \log (d / \varepsilon))$ time using multipole method.

Theorem 64 ([6, Sec 5]). Let $\phi_{i}(s)$ be the Lagrange basis polynomials on the Chebyshev nodes on $[0, \ell]$, namely, $\phi_{i}(s)=\prod_{j \neq i} \frac{s-c_{j}}{c_{i}-c_{j}}$ with $c_{i}=\frac{\ell}{2}+\frac{\ell}{2} \cos \left(\frac{2 i-1}{2 d} \pi\right)$. Given a polynomial $p(s)=\sum_{j} \alpha_{j} \phi_{j}(s)$ and a point set $\left\{x_{1}, x_{2}, \cdots, x_{d}\right\}$, one can compute $t_{i}$ such that

$$
\left|t_{i}-\int_{0}^{x_{i}} p(s) d s\right| \leq \varepsilon \ell \sqrt{\sum_{j \neq k}\left(\alpha_{j}-\alpha_{k}\right)^{2}} \text { for } i \in[d]
$$

in time $O(d \log (d / \varepsilon))$.

Now we have everything to state our main result in this subsection.

Theorem 65. Let $u(t) \in \mathbb{R}^{n}$ be the solution of the $O D E$ (5.1). Suppose we are given $\varepsilon, \ell>0$ and $1 \leq p \leq \infty$ such that

1. There is a degree $d$ polynomial $q$ from $\mathbb{R}$ to $\mathbb{R}^{n}$ such that $q(0)=v$ and $\left\|\frac{d}{d t} u(t)-\frac{d}{d t} q(t)\right\|_{p} \leq \frac{\varepsilon}{\ell}$ for all $0 \leq t \leq \ell$.

2. We have that $\|F(x, t)-F(y, t)\|_{p} \leq \frac{1}{2000 \ell}\|x-y\|_{p}$ for all $\|x-v\|_{p} \leq K$ and $\|y-v\|_{p} \leq K$ with $K=4000 \ell \max _{t \in[0, \ell]}\|F(v, t)\|_{p}$.

Then, Algorithm CollocationMethod outputs a degree d polynomial $p(t)$ such that $\max _{0 \leq t \leq \ell}\|u(t)-p(t)\|_{p} \leq$ $4003 \varepsilon$ in $O\left(n d \log ^{2}(d K / \varepsilon)\right)$ time and $O(d \log (K / \varepsilon))$ evaluations of $F$. 
Proof. First, we estimate the initial error. Let $\zeta^{(\infty)}$ be the solution to $(5.3), \zeta_{i}^{(0)}=v+T(\bar{v})_{i}$ be the initial vector and $\bar{v}=(v, v, \cdots, v) \in \mathbb{R}^{d \times n}$. Then, we have that

$$
\begin{aligned}
\left\|\zeta^{(\infty)}-\zeta^{(0)}\right\|_{\infty ; p} & =\max _{i}\left\|\left(v+T\left(\zeta^{(\infty)}\right)_{i}\right)-\left(v+T(\bar{v})_{i}\right)\right\|_{p} \\
& =\left\|T\left(\zeta^{(\infty)}\right)-T(\bar{v})\right\|_{\infty ; p} \\
& \leq \frac{1}{2}\left\|\zeta^{(\infty)}-\bar{v}\right\|_{\infty ; p} \\
& \leq \frac{1}{2}\left(\left\|\zeta^{(\infty)}-\zeta^{(0)}\right\|_{\infty ; p}+\left\|\zeta^{(0)}-\bar{v}\right\|_{\infty ; p}\right) .
\end{aligned}
$$

Therefore, we have that

$$
\begin{aligned}
\left\|\zeta^{(\infty)}-\zeta^{(0)}\right\|_{\infty ; p} & \leq\left\|\zeta^{(0)}-\bar{v}\right\|_{\infty ; p} \\
& =\|T((v, v, \cdots, v))\|_{\infty ; p} \\
& \leq 1000 \ell \max _{i}\left\|F\left(v, c_{i}\right)\right\|_{p} \\
& \leq \frac{K}{4}
\end{aligned}
$$

Hence, we have that $\left\|\zeta^{(\infty)}-\bar{v}\right\|_{\infty ; p} \leq \frac{K}{2}$.

Using the assumption on $F$, Lemma 63 shows that $\|T(x)-T(y)\|_{\infty ; p} \leq \frac{1}{2}\|x-y\|_{\infty ; p}$ and hence

$$
\left\|\zeta^{(\infty)}-\zeta^{(k)}\right\|_{\infty ; p} \leq \frac{K}{2^{k+1}}
$$

Thus, it takes $\log _{2}(K / \varepsilon)$ iteration to get a point $\zeta^{(k)}$ with

$$
\left\|\zeta^{(\infty)}-\zeta^{(k)}\right\|_{\infty ; p} \leq \varepsilon
$$

Also, this shows that $\left\|\zeta^{(\infty)}-\bar{v}\right\|_{\infty ; p} \leq K$. Hence, we only requires the assumption (2) to be satisfied in this region.

Now, we show that $\zeta^{(k)}$ is close to the solution by using the existence of $q$. By the assumption on $q$, we have that $\left\|\frac{d}{d t} u(t)-\frac{d}{d t} q(t)\right\|_{p} \leq \frac{\varepsilon}{\ell}$ and hence $\|u(t)-q(t)\|_{p} \leq \varepsilon$. Using the smoothness of $F$, we have that $\|F(u(t), t)-F(q(t), t)\|_{p} \leq \frac{\varepsilon}{\ell}$ for all $0 \leq t \leq \ell$. Therefore, we have that

$$
\begin{aligned}
\left\|\frac{d}{d t} q(t)-F(q(t), t)\right\|_{p} & \leq\left\|\frac{d}{d t} q(t)-\frac{d}{d t} u(t)\right\|_{p}+\|F(q(t), t)-F(u(t), t)\|_{p} \\
& \leq 2 \frac{\varepsilon}{\ell}
\end{aligned}
$$

for all $0 \leq t \leq 1$. Therefore, we have

$$
\begin{aligned}
q(t) & =v+\int_{0}^{t} \sum_{j=1}^{d} \frac{d}{d t} q\left(c_{j}\right) \phi_{j}(s) d s \\
& =v+\int_{0}^{t} \sum_{j=1}^{d}\left(F\left(q\left(c_{j}\right), c_{j}\right)+\delta_{j}\right) \phi_{j}(s) d s
\end{aligned}
$$


where $\left\|\delta_{i}\right\|_{p} \leq 2 \frac{\varepsilon}{\ell}$ for all $i \in[d]$. By Lemma 63, we have that

$$
\left\|q(t)-v-\int_{0}^{t} \sum_{j=1}^{d} F\left(q\left(c_{j}\right), c_{j}\right) \phi_{j}(s) d s\right\|_{p} \leq 2000 \varepsilon .
$$

Now, we compare $\bar{q}_{j} \stackrel{\text { def }}{=} q\left(c_{j}\right)$ with the approximate solution constructed by the fix point algorithm

$$
q^{(k)}(t)=v+\int_{0}^{t} \sum_{j=1}^{d} F\left(\zeta_{j}^{(k)}, c_{j}\right) \phi_{j}(s) d s .
$$

For $0 \leq t \leq \ell$, we have

$$
\begin{aligned}
\left\|q(t)-q^{(k)}(t)\right\|_{p} & \leq\left\|T(\bar{q})-T\left(\zeta^{(k)}\right)\right\|_{\infty ; p}+2000 \varepsilon \\
& \leq \frac{1}{2}\left\|\bar{q}-\zeta^{(k)}\right\|_{\infty ; p}+2000 \varepsilon
\end{aligned}
$$

Setting $t=c_{i}$, we have

$$
\begin{aligned}
\left\|\bar{q}-\zeta^{(k+1)}\right\|_{\infty ; p} & =\max _{i}\left\|\bar{q}\left(c_{i}\right)-q^{(k)}\left(c_{i}\right)\right\|_{p} \\
& \leq \frac{1}{2}\left\|q-\zeta^{(k)}\right\|_{\infty ; p}+2000 \varepsilon
\end{aligned}
$$

Since $\left\|\zeta^{(k+1)}-\zeta^{(k)}\right\|_{\infty ; p} \leq\left\|\zeta^{(k+1)}-\zeta^{(\infty)}\right\|_{\infty ; p}+\left\|\zeta^{(k)}-\zeta^{(\infty)}\right\|_{\infty ; p} \leq 2 \varepsilon$, we have

$$
\left\|\bar{q}-\zeta^{(k)}\right\|_{\infty ; p} \leq \frac{1}{2}\left\|\bar{q}-\zeta^{(k)}\right\|_{\infty ; p}+2 \varepsilon+2000 \varepsilon
$$

Therefore, we have

$$
\left\|\bar{q}-\zeta^{(k)}\right\|_{\infty ; p} \leq 4004 \varepsilon
$$

Putting it into (5.5), we have

$$
\left\|q(t)-q^{(k)}(t)\right\|_{p} \leq 4002 \varepsilon
$$

for all $0 \leq t \leq \ell$. Using that $\|u(t)-q(t)\|_{p} \leq \varepsilon$, we have

$$
\left\|u(t)-q^{(k)}(t)\right\|_{p} \leq 4003 \varepsilon
$$

Hence, it proves the guarantee.

Each iteration involves computing $v_{k}+\int_{0}^{c_{i}} \sum_{j=1}^{d} F\left(\zeta_{j}^{(z)}, c_{j}\right)_{k} \phi_{j}(s) d s$ for all $i \in[d], k \in[n]$. Note that $\sum_{j=1}^{d} F\left(\zeta_{j}^{(z)}, c_{j}\right)_{k} \phi_{j}(s)$ is a polynomial expressed by Lagrange polynomials. Theorem 64 shows they can be computed in $O(d \log (d K / \varepsilon)))$ with $\frac{\varepsilon}{K d^{O(1)}}$ accuracy. Since there are $n$ coordinates, it takes $O(n d \log (d K / \varepsilon)))$ time plus $d$ evaluation per iteration.

The theorem above essentially says that if the solution is well approximated by a polynomial and if the $F$ has small enough Lipschitz constant, then we can reconstruct the solution efficiently. Note that this method is not useful for stochastic differential equation because Taylor expansion of the solution involves the high moments of probability distributions which is very expensive to store.

The assumption on the Lipschitz constant of $F$ holds for our application. For the rest of this subsection, we show that this assumption is not necessary by taking multiple steps. This is mainly to make the result easier to use for other applications and not needed for this paper. First, we prove that the collocation method is stable under small perturbation of the initial solution. 
Lemma 66. Let $p(t)$ and $\tilde{p}(t)$ be the outputs of CollocationMethod for the initial value $v$ and the initial value $\tilde{v}$. We make the same assumption as Theorem 65 for the initial condition $v$ (albeit a small change in the constants). Suppose that $\|\tilde{v}-v\|_{p} \leq c \ell \max _{t \in[0, \ell]}\|F(v, t)\|_{p}$ with small enough constant $c$, we have that

$$
\max _{0 \leq t \leq \ell}\|p(t)-\tilde{p}(t)\|_{p}=O\left(\|\tilde{v}-v\|_{p}\right) .
$$

Proof. With a proof similar to Theorem 65, we know that $T$ is $\frac{1}{2}$ Lipschitz. Here, we use that $F$ is Lipschitz in a neighbour of $v$ and a neighbour of $\tilde{v}$. Since $\tilde{v}$ is close to $v$, we only need to make the assumption on a neighbour of $v$.

Let $\zeta^{(k)}, \tilde{\zeta}^{(k)}$ be the corresponding intermediate variables in the algorithm. Since $\zeta_{i}^{(0)}=$ $v+T(v, v, \cdots)_{i}$ and $\tilde{\zeta}_{i}^{(0)}=\tilde{v}+T(\tilde{v}, \tilde{v}, \cdots)_{i}$, we have that

$$
\begin{aligned}
\left\|\tilde{\zeta}^{(0)}-\zeta^{(0)}\right\|_{\infty ; p} & \leq\|\tilde{v}-v\|_{p}+\|T(\tilde{v}, \tilde{v}, \cdots)-T(v, v, \cdots)\|_{\infty ; p} \\
& \leq\|\tilde{v}-v\|_{p}+\frac{1}{2}\|\tilde{v}-v\|_{p}=\frac{3}{2}\|\tilde{v}-v\|_{p} \leq 2\|\tilde{v}-v\|_{p} .
\end{aligned}
$$

Since $\zeta_{i}^{(k+1)}=v+T\left(\zeta^{(k)}\right)_{i}$ and $\tilde{\zeta}_{i}^{(k+1)}=\tilde{v}+T\left(\tilde{\zeta}^{(k)}\right)_{i}$, by induction, we have that

$$
\begin{aligned}
\left\|\tilde{\zeta}^{(k+1)}-\zeta^{(k+1)}\right\|_{\infty ; p} & \leq\|\tilde{v}-v\|_{p}+\frac{1}{2}\left\|\tilde{\zeta}^{(k)}-\zeta^{(k)}\right\|_{\infty ; p} \\
& \leq\|\tilde{v}-v\|_{p}+\frac{1}{2} \cdot 2\|\tilde{v}-v\|_{p} \leq 2\|\tilde{v}-v\|_{p} .
\end{aligned}
$$
that

Now, we note that $p(t)=v+\int_{0}^{t} \sum_{j=1}^{d} F\left(\zeta_{j}^{(Z+1)}, c_{j}\right) \phi_{j}(s) d s$. By our assumption on $F$, we have

$$
\begin{aligned}
\left\|F\left(\zeta_{j}^{(Z+1)}, c_{j}\right)-F\left(\tilde{\zeta}_{j}^{(Z+1)}, c_{j}\right)\right\|_{p} & =O\left(\frac{1}{\ell}\right)\left\|\zeta_{j}^{(Z+1)}-\tilde{\zeta}_{j}^{(Z+1)}\right\|_{p} \\
& =O\left(\frac{1}{\ell}\right)\|\tilde{v}-v\|_{p} .
\end{aligned}
$$

Apply second part of Lemma 63, we have that

$$
\|p(t)-\tilde{p}(t)\|_{p}=O\left(\|\tilde{v}-v\|_{p}\right)
$$

for any $0 \leq t \leq \ell$.

Now, we give the main theorem of this subsection.

Theorem 67. Let $u(t) \in \mathbb{R}^{n}$ be the solution of the $O D E$ (5.1). Suppose we are given $\varepsilon>0$ and $1 \leq p \leq \infty$ such that

1. There is a degree $d$ polynomial $q$ from $\mathbb{R}$ to $\mathbb{R}^{n}$ such that $q(0)=v$ and $\left\|\frac{d}{d t} u(t)-\frac{d}{d t} q(t)\right\|_{p} \leq \varepsilon$ for all $0 \leq t \leq 1$.

2. For some $L \geq 1$, we have that $\|F(x, t)-F(y, t)\|_{p} \leq L\|x-y\|_{p}$ for all $x, y$ and $0 \leq t \leq 1$.

Then, we can compute $u$ such that $\|u-u(1)\|_{p}=O(\varepsilon)$ in $O\left(n d L^{3} \log ^{2}(d K / \varepsilon)\right)$ time and $O\left(d L^{2} \log (K / \varepsilon)\right)$ evaluations of $F$ where $K=\max _{x, 0 \leq t \leq 1}\|F(x, t)\|_{p}$. 
Proof. Let $\ell=\frac{1}{2000 L}$. By Theorem 65, we can compute $u(\ell)$ in $O\left(n d \log ^{2}(d K / \varepsilon)\right)$ time and $O(d \log (K / \varepsilon))$ evaluations of $F$. Now, we can apply 65 again with our approximate of $u(\ell)$ to get $u(2 \ell)$. Repeating this process, we can compute $u(1)$.

Lemma 66 shows that if the initial value of $u(0)$ has $\delta$ error, the collocation method would output $u(\ell)$ with $O(\delta)+\varepsilon$ error. Therefore, to compute $u(1)$ with $\varepsilon$ error, we need to iteratively compute $y(i \ell)$ with error $\varepsilon / \Theta(1)^{1 / \ell-i}$. Hence, the total running time is

$$
\sum_{i=1}^{1 / \ell} O\left(n d \log ^{2}\left(\Theta(1)^{1 / \ell-i} d K / \varepsilon\right)\right)=O\left(n d\left(\frac{1}{\ell}\right)^{3} \log ^{2}(d K / \varepsilon)\right)
$$

with

$$
\sum_{i=1}^{1 / \ell} O\left(d \log \left(\Theta(1)^{1 / \ell-i} d K / \varepsilon\right)\right)=O\left(d\left(\frac{1}{\ell}\right)^{2} \log ^{2}(d K / \varepsilon)\right)
$$

evaluations of $F$.

Remark 68. The main bottleneck of this algorithm is that the error blows up exponentially when we iteratively apply the collocation method. We believe this can be alleviated by using the collocation method in one shot but with a more smart initialization. Since we do not face this problem for our main application, we left this as a future investigation.

\subsection{Second Order ODE}

Now, we consider the following second order ODE

$$
\begin{aligned}
\frac{d^{2}}{d t^{2}} u(t) & =F\left(\frac{d}{d t} u(t), u(t), t\right), \text { for } 0 \leq t \leq \ell \\
\frac{d}{d t} u(0) & =w, \\
u(0) & =v
\end{aligned}
$$

where $F: \mathbb{R}^{2 n+1} \rightarrow \mathbb{R}^{n}$ and $u(t) \in \mathbb{R}^{n}$. Using a standard reduction from second order ODE to first order ODE, we show how to apply our first order ODE method to second order ODE.

Theorem 69. Let $x(t) \in \mathbb{R}^{n}$ be the solution of the $O D E$ (5.6). Given some $\varepsilon, \ell>0$ and $1 \leq p \leq \infty$, let $\alpha=4000 \ell$ and suppose that

1. There is a degree d polynomial $q$ from $\mathbb{R}$ to $\mathbb{R}^{n}$ such that $q(0)=v, q^{\prime}(0)=w,\left\|\frac{d}{d t^{2}} x(t)-\frac{d}{d t^{2}} q(t)\right\|_{p} \leq$ $\frac{\varepsilon}{\ell^{2}}$ for all $0 \leq t \leq \ell$.

2. We have that $\|F(x, \gamma, t)-F(y, \eta, t)\|_{p} \leq \frac{1}{\alpha}\|x-y\|_{p}+\frac{1}{\alpha^{2}}\|\gamma-\eta\|_{p}$ for all $\|x-w\|_{p} \leq K$, $\|y-w\|_{p} \leq K,\|\gamma-v\|_{p} \leq \alpha K,\|\eta-v\|_{p} \leq \alpha K$ where $K=\alpha \max _{t \in[0, \ell]}\|F(w, v, t)\|_{p}+\|w\|_{p}$.

Then, in $O\left(n d \log ^{2}(d K / \varepsilon)\right)$ time plus $O(d \log (K / \varepsilon))$ evaluations of $F$, we can find $p(t)$ such that

$$
\max _{0 \leq t \leq \ell}\|u(t)-p(t)\|_{p}=O(\varepsilon) \quad \text { and } \max _{0 \leq t \leq \ell}\left\|u^{\prime}(t)-p^{\prime}(t)\right\|_{p}=O(\varepsilon / \ell)
$$

Proof. Let $\alpha=4000 \ell$. Let $x(t)=\left(\alpha u^{\prime}(\alpha t), u(\alpha t)\right) \in \mathbb{R}^{2 n}$. Note that $x(t)$ satisfies the following ODE

$$
\begin{aligned}
\frac{d}{d t} x(t) & =\bar{F}(x(t), t) \text { for } 0 \leq t \leq \ell \\
x(0) & =(\alpha w, v)
\end{aligned}
$$


where $\bar{F}(x(t), t)=\left(\alpha^{2} F\left(\alpha^{-1} x_{(1)}(t), x_{(2)}(t), \alpha t\right), x_{(1)}(t)\right), x_{(1)}(t)$ is the first $n$ variables of $x(t)$ and $x_{(2)}(t)$ is the last $n$ variables of $x(t)$. Next, we verify the conditions of Theorem 65 for this ODE. Let $\bar{\ell}=\frac{1}{6000}$ and $\bar{K}=4000 \bar{\ell} \max _{t \in[0, \ell]}\|\bar{F}(x(0), t)\|_{p}$. Note that

$$
\bar{K} \leq \alpha^{2} \max _{t \in[0, \ell]}\|F(w, v, t)\|_{p}+\alpha\|w\|_{p} .
$$

For any $y, z$ such that $\|y-x(0)\|_{p} \leq \bar{K}$ and $\|z-x(0)\|_{p} \leq \bar{K}$, we apply the assumption on $F$ and get that

$$
\begin{aligned}
& \|\bar{F}(y, t)-\bar{F}(z, t)\|_{p} \\
\leq & \alpha^{2}\left\|F\left(\alpha^{-1} y_{(1)}, y_{(2)}, \alpha t\right)-F\left(\alpha^{-1} z_{(1)}, z_{(2)}, \alpha t\right)\right\|_{p}+\left\|y_{(1)}-z_{(1)}\right\|_{p} \\
\leq & \alpha^{2}\left(\frac{1}{\alpha}\left\|\alpha^{-1} y_{(1)}-\alpha^{-1} z_{(1)}\right\|_{p}+\frac{1}{\alpha^{2}}\left\|y_{(2)}-z_{(2)}\right\|_{p}\right)+\left\|y_{(1)}-z_{(1)}\right\|_{p} \\
\leq & 3\|y-z\|_{p} .
\end{aligned}
$$

Also, by our assumption on $x$, we have a polynomial $\bar{q}=\left(\alpha q^{\prime}(\alpha t), q(\alpha t)\right)$ such that

$$
\left\|\frac{d}{d t} x(t)-\frac{d}{d t} \bar{q}(t)\right\|_{p}=O\left(\begin{array}{l}
\varepsilon \\
\bar{\ell}
\end{array}\right)
$$

where $x$ is the solution of the ODE (5.7). Therefore, Theorem 65 shows that we can compute $x$ with $O(\varepsilon)$ error in $O\left(n d \log ^{2}(d K / \varepsilon)\right)$ time plus $O(d \log (K / \varepsilon))$ evaluations of $F$. Since $x=\left(\alpha u^{\prime}, u\right)$, this gives us an approximate of $u$ with error $O(\varepsilon)$ and $u^{\prime}$ with error $O(\varepsilon / \ell)$.

\subsection{Example: Discretization of Physarum dynamics}

In this section, we use the Physarum dynamics as an example to showcase the usefulness of the collocation method. We consider a linear program of the form

$$
\min _{A x=b, x \geq 0} c^{T} x
$$

where $A \in \mathbb{Z}^{m \times n}, c \in \mathbb{Z}_{>0}^{n}$ and $b \in \mathbb{Z}^{m}$. Inspired by Physarum polycephalum (a slime mold), Straszak and Vishnoi [34] introduce the following dynamics for solving the linear program:

$$
\frac{d x}{d t}=W A^{T}(A W A)^{-1} b-x
$$

where $W$ is a diagonal matrix with the diagonal $W_{i i}=x_{i} / c_{i}$. In this subsection, we follow their notations/assumptions:

1. Assume that $A$ is full rank and this linear program has a feasible solution.

2. Let OPT be the optimal value of the linear program (5.8).

3. Let $D$ be the maximum sub-determinant of $A$, i.e. $D=\max _{A^{\prime}}$ is a square submatrix of $A\left|\operatorname{det}\left(A^{\prime}\right)\right|$. For graph problems, $A$ is usually an unimodular matrix and hence $D=1$.

4. Assume that we have an initial point $x(0)$ such that $A x(0)=b$ and a parameter $M$ such that

$$
M^{-1} \leq x(0) \leq M \text { and } c^{T} s \leq M \cdot \text { OPT } .
$$


They showed that the continuous dynamics converges linearly to the solution:

Lemma 70 (Convergence of Physarum dynamics, [34, Thm 6.3]). Consider $x(t)$ be the solution of 5.9 with initial point $A x(0)=b$. Then, we have that

$$
O P T \leq c^{T} x(t) \leq O P T+(n+M)^{2} e^{8 D^{2}\|c\|_{1}\|b\|_{1}-D^{-3} t} .
$$

Furthermore, they analyzed the Euler method for this dynamics and obtained the following result:

Lemma 71 (Euler method for Physarum dynamics, [34, Thm 7.1]). Consider the discretization of the Physarum dynamics

$$
\begin{aligned}
x^{(k+1)} & =(1-h) x^{(k)}+h W^{(k)} A^{T}\left(A W^{(k)} A\right)^{-1} b, \\
x^{(0)} & =x(0)
\end{aligned}
$$

with the diagonal matrix $W_{i i}^{(k)}=x_{i}^{(k)} / c_{i}$. Then, for any $\varepsilon>0$ and $h=\frac{1}{6} \varepsilon\|c\|_{1}^{-2} D^{-2}$, we have that

$$
O P T \leq c^{T} x^{(k)} \leq(1+\varepsilon) O P T
$$

where $k=O\left(\frac{\ln M}{\varepsilon^{2} h^{2}}\right)=O\left(\frac{\|c\|_{1}^{4} D^{4} \ln M}{\epsilon^{4}}\right)$.

Note that the continuous process converges linearly while the discrete process converges sublinearly. In this section, we show how to use collocation method to get a discrete process that converges "linearly".

Lemma 72 (Collocation method for Physarum dynamics). For any $T \geq 0$ and $1>\varepsilon>0$, we can compute $y$ such that

$$
(1-\varepsilon) x_{i}(T) \leq y_{i} \leq(1+\varepsilon) x_{i}(T)
$$

for all $i$ in time $O\left(n^{\omega+3} D^{6}\|c\|_{1}^{3} T^{3} \log ^{2}\left(n D\|c\|_{1} / \varepsilon\right)\right)$.

Proof. Let $y=\ln x$. The Physarum dynamics can be written as

$$
\begin{aligned}
\frac{d y}{d t} & =\operatorname{diag}\left(\frac{1}{c}\right) A^{T}\left(A \operatorname{diag}\left(\frac{e^{y}}{c}\right) A^{T}\right)^{-1} b-1 \\
& \stackrel{\text { def }}{=} F(y) .
\end{aligned}
$$

To use Theorem 65, we need to bound the Lipschitz constant of $F$ and show that $y(t)$ can be approximated by polynomial.

The Lipschitz constant of $F$ : Given any path $\tilde{y}(t)$ in $\mathbb{R}^{n}$. Let $W(t)=\operatorname{diag}\left(\frac{e^{\tilde{y}(t)}}{c}\right), C=\operatorname{diag}(c)$ and $P(t)=A^{T}\left(A W(t) A^{T}\right)^{-1} A W(t)$. Then, we have that

$$
\begin{aligned}
\frac{d}{d t} F(\tilde{y}(t)) & =\frac{d}{d t} C^{-1} A^{T}\left(A W(t) A^{T}\right)^{-1} b \\
& =-C^{-1} A^{T}\left(A W(t) A^{T}\right)^{-1} A W(t) \operatorname{diag}\left(\frac{d \tilde{y}}{d t}\right) A^{T}\left(A W(t) A^{T}\right)^{-1} b \\
& =-C^{-1} P(t) \operatorname{diag}\left(\frac{d \tilde{y}}{d t}\right) P(t) c
\end{aligned}
$$


where we used $A x=b$ at the last line. By [34, Lem 5.2], we have that

$$
\|P(t)\|_{1 \rightarrow \infty} \leq D
$$

Hence, we have that

$$
\begin{aligned}
\left\|P(t) \operatorname{diag}\left(\frac{d \tilde{y}}{d t}\right) P(t) c\right\|_{\infty} & \leq D\left\|\operatorname{diag}\left(\frac{d \tilde{y}}{d t}\right) P(t) c\right\|_{1} \\
& \leq n D\left\|\frac{d \tilde{y}}{d t}\right\|_{\infty}\|P(t) c\|_{\infty} \leq n D^{2}\left\|\frac{d \tilde{y}}{d t}\right\|_{\infty}\|c\|_{1} .
\end{aligned}
$$

Since $c$ is integral, we have that

$$
\left\|\frac{d}{d t} F(\tilde{y}(t))\right\|_{\infty} \leq n D^{2}\left\|\frac{d \tilde{y}}{d t}\right\|_{\infty}\|c\|_{1} .
$$

Hence, $F$ has Lipschitz constant $n D^{2}\|c\|_{1}$ in $L^{\infty}$ norm.

Analyticity of $y(t)$ : Note that

$$
\frac{d y}{d t}=C^{-1} P(t) c-1
$$

By (5.10), we have that $\left\|\frac{d y}{d t}\right\|_{\infty} \leq 1+D\|c\|_{1} \leq 2 D\|c\|_{1}$. Note that

$$
\frac{d}{d t} P(t)=-P(t) \operatorname{diag}\left(\frac{d y}{d t}\right) P(t)+P(t) \operatorname{diag}\left(\frac{d y}{d t}\right) .
$$

Hence, we have that

$$
\frac{d^{2} y}{d t^{2}}=-C^{-1} P(t) \operatorname{diag}\left(\frac{d y}{d t}\right) P(t) c+C^{-1} P(t) \operatorname{diag}\left(\frac{d y}{d t}\right) c
$$

and

$$
\begin{aligned}
\left\|\frac{d^{2} y}{d t^{2}}\right\|_{\infty} & \leq n D\left\|\frac{d y}{d t}\right\|_{\infty} D\|c\|_{1}+D\left\|\frac{d y}{d t}\right\|_{\infty}\|c\| \\
& \leq 2 n D^{2}\|c\|_{1}\left\|\frac{d y}{d t}\right\|_{\infty} \leq 4 n D^{3}\|c\|_{1}^{2} .
\end{aligned}
$$

By induction, one can show that

$$
\left\|\frac{d^{k} y}{d t^{k}}\right\|_{\infty}=O(1)^{k} k ! D^{2 k-1} n^{k-1}\|c\|_{1}^{k} .
$$

For $d=\log (1 / \varepsilon)$ and $\ell=\frac{1}{\Omega\left(n D^{2}\|c\|_{1}\right)}$, we have that

$$
\left\|y(t)-\sum_{k=0}^{d-1} \frac{1}{k !} y^{(k)}(0) t^{k}\right\|_{\infty} \leq O(1)^{d} D^{2 d-1} n^{d-1}\|c\|_{1}^{d} \ell^{d} \leq \varepsilon
$$

for any $0 \leq t \leq \ell$. Similarly, we have that

$$
\left\|\frac{d}{d t} y(t)-\frac{d}{d t} \sum_{k=0}^{d-1} \frac{1}{k !} y^{(k)}(0) t^{k}\right\|_{\infty} \leq \varepsilon
$$


for any $0 \leq t \leq \ell$.

Since $F$ has Lipschitz constant $n D^{2}\|c\|_{1}$, we can apply Theorem 65 with $\ell=O\left(\frac{1}{n D^{2}\|c\|_{1} \log (1 / \varepsilon)}\right)$, $d=\log (1 / \varepsilon), p=\infty$. Hence, we can compute $y(\ell)$ with $\varepsilon$ error in $O\left(n \log ^{2}\left(n D\|c\|_{1} / \varepsilon\right)\right)$ time with $O\left(\log \left(n D\|c\|_{1} / \varepsilon\right)\right)$ evaluations of $F$. Since $F$ can be computed in matrix multiplication time, we can compute $y(\ell)$ with $\varepsilon$ error in $O\left(n^{\omega} \log ^{2}\left(n D\|c\|_{1} / \varepsilon\right)\right)$ time.

Next, we note that if the initial value of $y(0)$ has $\delta$ error, the collocation method would output $y(\ell)$ with $O(\delta)+\varepsilon$ error. Therefore, to compute $y(T)$ with $\varepsilon$ error, we need to iteratively compute $y(i \ell)$ with error $\varepsilon / \Theta(1)^{T / \ell-i}$. Hence, the total running time is

$$
\sum_{i=1}^{T / \ell} O\left(n^{\omega} \log ^{2}\left(\Theta(1)^{T / \ell-i} n D\|c\|_{1} / \varepsilon\right)\right)=O\left(n^{\omega}\left(\frac{T}{\ell}\right)^{3} \log ^{2}\left(n D\|c\|_{1} / \varepsilon\right)\right) .
$$

Remark 73. We use this example merely to showcase that the collocation method is useful for getting a polynomial time algorithm for solving ordinary differential equations. The $k^{\text {th }}$ order derivatives of the path $x(t)$ can be computed efficiently in $(k n)^{O(1)}$ time and hence one can simply use Taylor series to approximate Physarum dynamics. Alternatively, since the path $x(t)$ is the solution of certain convex optimization problem [34, Thm 4.2], we can be computed it directly in $\tilde{O}\left(n^{3}\right)$ time [19].

\subsection{Derivative Estimations}

For any smooth one dimension function $f$, we know by Taylor's theorem that

$$
f(x)=\sum_{k=0}^{N} \frac{f^{(k)}(a)}{k !}(x-a)^{k}+\frac{1}{N !} \int_{a}^{x}(x-t)^{N} f^{(N+1)}(t) d t .
$$

This formula provides a polynomial estimate of $f$ around $a$. To analyze the accuracy of this estimate, we need to bound $\left|f^{(N+1)}(t)\right|$. In one dimension, we could simply give explicit formulas for the derivatives of $f$ and use it to estimate the remainder term. However, for functions in high dimension, it is usually too tedious. Here we describe some techniques for bounding the derivatives of higher dimensional functions.

The derivatives of one-variable functions can be bounded via Cauchy's estimate (Theorem 19). In Section 5.5, we give calculus rules that reduces the problem of estimating derivatives of highdimensional functions to derivatives of one-dimensional functions. In Section 5.6, we show how to reduce bounding the derivative for an arbitrary ODE to an ODE in one dimension.

\subsection{Explicit Function}

In this subsection, we show how to bound the derivatives of a complicated explicit function using the following object, generating upper bound. We reduce estimates of the derivatives of functions in high dimension to one variable rational functions. Since rational functions are holomorphic, one can apply Cauchy's estimates (Theorem (19)) to bound their derivatives.

Definition 74. Given a function $F$. We call that $F \leq_{x} f$ for some one variable function $f: \mathbb{R} \rightarrow \mathbb{R}$ if

$$
\left\|D^{(k)} F(x)\left[\Delta_{1}, \Delta_{2}, \cdots, \Delta_{k}\right]\right\| \leq f^{(k)}(0) \prod_{i=1}^{k}\left\|\Delta_{i}\right\|^{k}
$$

for any $k \geq 0$ and any $\Delta_{i}$. 
Remark. In general, $F \leq_{x} f$ and $f(t) \leq g(t)$ point-wise does NOT imply $F \leq_{x} g$. However, $F \leq_{x} f$ and $f \leq_{0} g$ does imply $F \leq_{x} g$.

Remark. The bounds we give in this subsection only assume that $\|\cdot\|$ is a norm and it satisfies $\|a b\| \leq\|a\|\|b\|$. In the later sections, we always use $\|\cdot\|_{2}$ for both matrix and scalar.

This concept is useful for us to reduce bounding derivatives of a high dimension function to bounding derivatives of 1 dimension function. First of all, we note that upper bounds are composable.

Lemma 75. Given $F \leq_{x} f$ and $G \leq_{F(x)} g$, we have that

$$
G \circ F \leq_{x} g \circ \bar{f}
$$

where $\bar{f}(s)=f(s)-f(0)$.

Proof. Fix any $\Delta_{1}, \Delta_{2}, \cdots$ be unit vectors in the domain of $F$. Let $H(x)=G \circ F(x)$. By chain rule, we have that

$$
\begin{aligned}
D H(x)\left[\Delta_{1}\right]= & D G(F(x))\left[D F(x)\left[\Delta_{1}\right]\right] \\
D H(x)\left[\Delta_{1}, \Delta_{2}\right]= & D G(F(x))\left[D^{2} F(x)\left[\Delta_{1}, \Delta_{2}\right]\right] \\
& +D^{2} G(F(x))\left[D F(x)\left[\Delta_{1}\right], D F(x)\left[\Delta_{2}\right]\right], \\
D H(x)\left[\Delta_{1}, \Delta_{2}, \Delta_{3}\right]= & D G(F(x))\left[D^{2} F(x)\left[\Delta_{1}, \Delta_{2}, \Delta_{3}\right]\right] \\
& +D^{2} G(F(x))\left[D^{2} F(x)\left[\Delta_{1}, \Delta_{2}\right], D F(x)\left[\Delta_{3}\right]\right] \\
& +D^{2} G(F(x))\left[D^{2} F(x)\left[\Delta_{1}, \Delta_{3}\right], D F(x)\left[\Delta_{2}\right]\right] \\
& +D^{2} G(F(x))\left[D^{2} F(x)\left[\Delta_{2}, \Delta_{3}\right], D F(x)\left[\Delta_{1}\right]\right] \\
& +D^{3} G(F(x))\left[D F(x)\left[\Delta_{1}\right], D F(x)\left[\Delta_{2}\right], D F(x)\left[\Delta_{3}\right]\right],
\end{aligned}
$$

Since $G \leq_{F(x)} g$, equation (5.13) shows that

$$
\begin{aligned}
\left\|D H(x)\left[\Delta_{1}\right]\right\| \leq & g^{(1)}(0)\left\|D F(x)\left[\Delta_{1}\right]\right\| \\
\left\|D^{2} H(x)\left[\Delta_{1}, \Delta_{2}\right]\right\| \leq & g^{(1)}(0)\left\|D^{2} F(x)\left[\Delta_{1}, \Delta_{2}\right]\right\| \\
& +g^{(2)}(0)\left\|D F(x)\left[\Delta_{1}\right]\right\|_{2}\left\|D F(x)\left[\Delta_{2}\right]\right\| \\
\left\|D^{3} H(x)\left[\Delta_{1}, \Delta_{2}, \Delta_{3}\right]\right\| \leq & g^{(1)}(0)\left\|D^{2} F(x)\left[\Delta_{1}, \Delta_{2}, \Delta_{3}\right]\right\| \\
& +g^{(2)}(0)\left\|D^{2} F(x)\left[\Delta_{1}, \Delta_{2}\right]\right\|\left\|D F(x)\left[\Delta_{3}\right]\right\| \\
& +g^{(2)}(0)\left\|D^{2} F(x)\left[\Delta_{1}, \Delta_{3}\right]\right\|\left\|D F(x)\left[\Delta_{2}\right]\right\| \\
& +g^{(2)}(0)\left\|D^{2} F(x)\left[\Delta_{2}, \Delta_{3}\right]\right\|\left\|D F(x)\left[\Delta_{1}\right]\right\| \\
\vdots & +g^{(3)}(0)\left\|D F(x)\left[\Delta_{1}\right]\right\|\left\|D F(x)\left[\Delta_{2}\right]\right\|\left\|D F(x)\left[\Delta_{3}\right]\right\| .
\end{aligned}
$$

Now, we use $F \leq_{x} f$ to get

$$
\begin{aligned}
\left\|D H(x)\left[\Delta_{1}\right]\right\| & \leq g^{(1)}(0) f^{(1)}(0)=(g \circ \bar{f})^{(1)}(0) \\
\left\|D^{2} H(x)\left[\Delta_{1}, \Delta_{2}\right]\right\| & \leq g^{(1)}(0) f^{(2)}(0)+g^{(2)}(0) f^{(1)}(0)^{2}=(g \circ \bar{f})^{(2)}(0), \\
\left\|D^{3} H(x)\left[\Delta_{1}, \Delta_{2}, \Delta_{3}\right]\right\| & \leq g^{(1)}(0) f^{(3)}(0)+2 g^{(2)}(0) f^{(2)}(0) f^{(1)}(0) \\
& \vdots+g^{(3)}(0) f^{(1)}(0)^{3}=(g \circ \bar{f})^{(3)}(0) .
\end{aligned}
$$


Therefore, we have that $\left\|D^{k} H(x)\left[\Delta_{i}\right]\right\| \leq(g \circ \bar{f})^{(k)}(0)$ for all $k \geq 1$. For $k=0$, we have that $\|H(x)\|=\|G(F(x))\| \leq g(0)=g(\bar{f}(0))$.

Next, we give some extra calculus rule for generating upper bounds.

Lemma 76. Given that $H_{i} \leq_{x} h_{i}$ for all $i=1, \cdots, k$. Then, we have that

$$
\sum_{i=1}^{k} H_{i} \leq_{x} \sum_{i=1}^{k} h_{i} \text { and } \prod_{i=1}^{k} H_{i} \leq_{x} \prod_{i=1}^{k} h_{i} .
$$

Given that $H \leq{ }_{x} h$ and $\left\|H^{-1}(x)\right\| \leq C$, we have that

$$
H^{-1} \leq_{x} \frac{1}{C^{-1}-(h(s)-h(0))} .
$$

Proof. Fix $\Delta_{1}, \Delta_{2}, \cdots$ be unit vectors. For the first claim, let $H=\sum H_{i}$, we note that

$$
D^{j} H\left[\Delta_{1}, \cdots, \Delta_{j}\right]=\sum_{i=1}^{k} D^{j} H_{i}\left[\Delta_{1}, \cdots, \Delta_{j}\right] .
$$

Therefore, we have that $\left\|D^{j} H\left[\Delta_{1}, \cdots, \Delta_{j}\right]\right\| \leq \sum_{i=1}^{k}\left\|D^{j} H_{i}\left[\Delta_{1}, \cdots, \Delta_{j}\right]\right\|$. Since $H \leq_{x} h_{i}$, we have that $\left\|D^{j} H\left[\Delta_{1}, \cdots, \Delta_{j}\right]\right\| \leq \sum_{i=1}^{k} h^{(j)}(0)$. Hence, we have $H \leq_{x} \sum_{i=1}^{k} h_{i}$.

For the second claim, we let $G=\prod_{i=1}^{k} H_{i}$ and note that

$$
D^{j} G=\sum_{i_{1}+i_{2}+\cdots+i_{k}=j} \prod_{l=1}^{k} D^{i_{l}} H_{l}
$$

Let $g=\prod_{i=1}^{k} h_{i}$. Then, we have that

$$
\left\|D^{j} G\right\| \leq \sum_{i_{1}+i_{2}+\cdots+i_{k}=j} \prod_{l=1}^{k}\left\|D^{i_{l}} H_{l}\right\| \leq \sum_{i_{1}+i_{2}+\cdots+i_{k}=j} \prod_{l=1}^{k} h_{i}^{\left(i_{l}\right)}(0)=D^{j} g(0) .
$$

Hence, $G \leq_{x} g$.

For the last claim, we first consider the function $\Phi(M)=M^{-1}$. Note that $D \Phi\left[\Delta_{1}\right]=-M^{-1} \Delta_{1} M^{-1}$, $D^{2} \Phi[\Delta]=M^{-1} \Delta_{1} M^{-1} \Delta_{2} M^{-1}+M^{-1} \Delta_{2} M^{-1} \Delta_{1} M^{-1}$ and hence

$$
\left\|D^{j} \Phi\right\| \leq j !\left\|M^{-1}\right\|^{j+1}=j ! C^{j+1}
$$

Hence, we have $\Phi \leq_{M} \sum_{j=0}^{\infty} \frac{j ! C^{j+1}}{j !} s^{j}=\frac{1}{C^{-1}-s}$. By Lemma 75, we see that $H^{-1} \leq_{x} \frac{1}{C^{-1}-(h(s)-h(0))}$.

\subsection{Explicit ODE}

In this section, we study the Taylor expansion of the solution of ODE (5.1).

Lemma 77. Let $u(t)$ be the solution of the $O D E u^{\prime}(t)=F(u(t))$. Suppose that $F \leq_{u(0)} f$ and let $\psi(t)$ be the solution of the $O D E \psi^{\prime}(t)=f(\psi(t))$ and $\psi(0)=0$. Then, we have

$$
\left\|u^{(k)}(0)\right\| \leq \psi^{(k)}(0)
$$

for all $k \geq 1$. 
Proof. Since $u^{\prime}(t)=F(u(t))$, we have that

$$
\begin{aligned}
u^{(2)}(t)= & D F(u(t))\left[u^{(1)}(t)\right], \\
u^{(3)}(t)= & D F(u(t))\left[u^{(2)}(t)\right]+D^{2} F(u(t))\left[u^{(1)}(t), u^{(1)}(t)\right], \\
u^{(4)}(t)= & D F(u(t))\left[u^{(3)}(t)\right]+2 D^{2} F(u(t))\left[u^{(2)}(t), u^{(1)}(t)\right] \\
& +D^{3} F(u(t))\left[u^{(1)}(t), u^{(1)}(t), u^{(1)}(t)\right]
\end{aligned}
$$

Therefore, we have

$$
\begin{aligned}
& \left\|u^{(2)}(0)\right\| \leq f^{(1)}(0)\left\|u^{(1)}(0)\right\|, \\
& \left\|u^{(3)}(0)\right\| \leq f^{(1)}(0)\left\|u^{(2)}(0)\right\|+f^{(2)}(0)\left\|u^{(1)}(0)\right\|^{2}, \\
& \left\|u^{(4)}(0)\right\| \leq f^{(1)}(0)\left\|u^{(3)}(0)\right\|+2 f^{(2)}(0)\left\|u^{(2)}(0)\right\|\left\|u^{(1)}(0)\right\|+f^{(3)}(0)\left\|u^{(1)}(0)\right\|^{3},
\end{aligned}
$$

By expanding $\psi^{\prime}(t)=f(\psi(t))$ at $t=0$, we see that

$$
\begin{aligned}
& \psi^{(2)}(0)=f^{(1)}(0) \psi^{(1)}(0) \\
& \psi^{(3)}(0)=f^{(1)}(0) \psi^{(2)}(0)+f^{(2)}(0)\left(\psi^{(1)}(0)\right)^{2} \\
& \psi^{(4)}(0)=f^{(1)}(0) \psi^{(3)}(0)+2 f^{(2)}(0) \psi^{(2)}(0) \psi^{(1)}(0)+f^{(2)}(0)\left(\psi^{(1)}(0)\right)^{3},
\end{aligned}
$$

Since $\left\|u^{(1)}(0)\right\|=\|F(u(0))\| \leq f(0)=\psi^{(1)}(0)$, we have that $\left\|u^{(k)}(0)\right\| \leq \psi^{(k)}(0)$ for all $k \geq 1$.

Now, we apply Lemma 77 to second order ODEs.

Lemma 78. Let $u(t)$ be the solution of the $O D E u^{\prime \prime}(t)=F\left(u^{\prime}(t), u(t), t\right)$. Given some $\alpha>0$ and define $\bar{F}(y, x, t)=\alpha^{2} F\left(\alpha^{-1} y(t)+u^{\prime}(0), x(t)+\alpha t u^{\prime}(0), \alpha t\right)$. Suppose that $\bar{F} \leq_{(0, u(0), 0)} f$ and let $\psi(t)$ be the solution of the ODE $\psi^{\prime}(t)=1+\psi(t)+f(\psi(t))$ and $\psi(0)=0$. Then, we have

$$
\left\|u^{(k)}(0)\right\| \leq \frac{\psi^{(k)}(0)}{\alpha^{k}}
$$

for all $k \geq 2$.

Proof. Let $x(t)=u(\alpha t)-\alpha t u^{\prime}(0)$ and $y(t)=\alpha u^{\prime}(\alpha t)-\alpha u^{\prime}(0)$. Then, we can write the problem into first order ODE

$$
\begin{aligned}
y^{\prime}(t) & =\alpha^{2} F\left(\alpha^{-1} y(t)+u^{\prime}(0), x(t)+\alpha t u^{\prime}(0), \alpha t\right)=\bar{F}(y(t), x(t), t), \\
x^{\prime}(t) & =y(t), \\
t^{\prime} & =1
\end{aligned}
$$

Let $\mathbf{F}(y, x, t)=(\bar{F}(y, x, t), y, 1)$. Then, we have that $\left\|D^{k} \mathbf{F}\right\| \leq\left\|D^{k} \bar{F}\right\|+\left\|D^{k} y\right\|+\left\|D^{k} 1\right\|$. Using $\bar{F} \leq_{(0, u(0), 0)} f$, we have that

$$
\mathbf{F} \leq_{(0, x(0), 0)} f+t+1
$$


By Lemma 77, we know that

$$
\left\|u^{(k)}(0)\right\|=\frac{\left\|x^{(k)}(0)\right\|}{\alpha^{k}} \leq \frac{\psi^{(k)}(0)}{\alpha^{k}} .
$$




\section{Implementation of Geodesic walk for log barrier}

\subsection{Complex Analyticity of geodesics, parallel transport and Jacobi fields}

The Cauchy-Kowalevski theorem (Theorem 20) shows that if a differential equation is complex analytic, then the equation has a unique complex analytic solution. As we see in section 4.1, the equations for geodesic, parallel transport and Jacobi field involve only rational functions. Since rational functions are complex analytic, the Cauchy-Kowalevski theorem shows that geodesics, orthogonal frames and Jacobi fields are also complex analytic.

Lemma 79. Geodesics, parallel transport and Jacobi fields are complex analytic for the Hessian manifold induced by the logarithmic barrier.

We bound the higher-order derivatives of geodesic, parallel transport and Jacobi field, using techniques developed in Section 5.4. Since these are complex analytic, this gives us a bound for their radius of convergence.

The purpose of these derivative bounds is to show that the solutions of the corresponding ODEs are well-approximated by low-degree polynomials, where the degree of the polynomial grows as $\log \left(\frac{1}{\epsilon}\right)$ for desired accuracy $\epsilon$. The bound on the degree also implies that the Collocation method for solving ODEs is efficient (roughly matrix multiplication time).

\subsubsection{Geodesic}

Motivated from the geodesic equation under Euclidean coordinate (4.1), we define the following auxiliary function

$$
\bar{F}_{\eta}(y, x, t)=\left(A_{x+t \eta}^{T} A_{x+t \eta}\right)^{-1} A_{x+t \eta}^{T} s_{x+t \eta, y+\eta}^{2} .
$$

The derivative bounds on geodesic rely on the smoothness of this auxiliary function.

Lemma 80. Under the normalization $A^{T} A=I, S_{x}=I$, we have that

$$
\bar{F}_{\eta}(y, x, t) \leq_{(0, x, 0)} \frac{3\left(\|A \eta\|_{4}+1\right)^{2}}{1-\max \left(8+8\|A \eta\|_{\infty}, 1\right) t}
$$

where $\bar{F}_{\eta}$ is defined in (6.1).

Proof. By the assumption that $S_{x}=I$, we have that $\left\|S_{x}\right\|_{2}=1$. Using $A^{T} A=I$, we have that

$$
\begin{aligned}
\left\|D S_{x+t \eta}(x, t)\left[\left(d_{x}, d_{t}\right)\right]\right\|_{2} & =\left\|\operatorname{Diag}\left(A d_{x}+d_{t} A \eta\right)\right\|_{2} \\
& \leq\left\|A d_{x}\right\|_{\infty}+\left\|d_{t} A \eta\right\|_{\infty} \\
& \leq\left\|d_{x}\right\|_{2}+\left|d_{t}\right|\|A \eta\|_{\infty} \\
& \leq\left(1+\|A \eta\|_{\infty}\right)\left\|\left(d_{x}, d_{t}\right)\right\|_{2} .
\end{aligned}
$$

Let $\beta=1+\|A \eta\|_{\infty}$. Then, we have that $S_{x+t \eta} \leq_{(x, t=0)} 1+\beta t$.

By using the inverse formula (Lemma 76),

$$
S_{x+t \eta}^{-1} \leq_{(x, 0)} \frac{1}{1-\beta t}
$$

Using $A^{T} A=I$, we have that $A \leq_{(x, 0)} 1$ and hence product formula (Lemma 76) shows that

$$
A_{x+t \eta}=S_{x+t \eta}^{-1} A \leq_{(x, 0)} \frac{1}{1-\beta t} .
$$


Since $A^{T} A=I$, we have that $A A^{T} \preceq I$ and hence $A_{x+t \eta}^{T} \leq_{(x, 0)} \frac{1}{1-\beta t}$. Therefore, we have that

$$
A_{x+t \eta}^{T} A_{x+t \eta} \leq(x, 0) \frac{1}{(1-\beta t)^{2}} .
$$

By using the inverse formula again,

$$
\left(A_{x+t \eta}^{T} A_{x+t \eta}\right)^{-1} \leq_{(x, 0)} \frac{1}{2-\frac{1}{(1-\beta t)^{2}}}=\frac{(1-\beta t)^{2}}{2(1-\beta t)^{2}-1} .
$$

Hence, we have that

$$
\left(A_{x+t \eta}^{T} A_{x+t \eta}\right)^{-1} A_{x+t \eta}^{T} \leq_{(x, 0)} \frac{(1-\beta t)^{2}}{2(1-\beta t)^{2}-1} \frac{1}{1-\beta t}=\frac{1-\beta t}{2(1-\beta t)^{2}-1} .
$$

Now, we consider the function $H(y)=(A(y+\eta))^{2}$. Note that

$$
\begin{aligned}
\|H(y)\|_{2} & \leq\|A(y+\eta)\|_{4}^{2}, \\
\|D H(y)[d]\|_{2} & =2\|(A(y+\eta)) A d\|_{2} \leq 2\|A(y+\eta)\|_{4}\|d\|_{2}, \\
\left\|D^{2} H(y)[d, d]\right\|_{2} & \leq 2\left\|(A d)^{2}\right\|_{2} \leq 2\|d\|_{2}^{2} .
\end{aligned}
$$

Therefore, we have that

$$
H \leq_{y=0}\|A \eta\|_{4}^{2}+2\|A \eta\|_{4} t+t^{2}=\left(\|A \eta\|_{4}+t\right)^{2} .
$$

Hence, we have that

$$
\bar{F}_{\eta}(y, x, t)=\left(A_{x+t \eta}^{T} A_{x+t \eta}\right)^{-1} A_{x+t \eta}^{T} s_{x+t \eta, y+\eta}^{2} \leq_{(0, x, 0)} \frac{(1-\beta t)\left(\|A \eta\|_{4}+t\right)^{2}}{2(1-\beta t)^{2}-1} \frac{1}{(1-\beta t)^{2}} .
$$

Let $\phi(t)=\frac{\left(\|A \eta\|_{4}+t\right)^{2}}{\left(2(1-\beta t)^{2}-1\right)(1-\beta t)}$ and we write $\phi(t)=\sum_{k=0}^{\infty} a_{k} t^{k}$. For any complex $|z|=\frac{1}{8} \min \left(\frac{1}{\beta},\|A \eta\|_{4}+8\right)$, we have that

$$
\begin{aligned}
|\phi(z)| & \leq \frac{\left(1-\frac{1}{8}\right)}{\left(2\left(1-\frac{1}{8}\right)^{2}-1\right)\left(1-\frac{1}{8}\right)^{2}}\left(\|A \eta\|_{4}+\frac{\|A \eta\|_{4}}{8}+1\right)^{2} \\
& \leq 3\left(\|A \eta\|_{4}+1\right)^{2}\left(\|A \eta\|_{4}+1\right)^{2} .
\end{aligned}
$$

Theorem 19 shows that

$$
\begin{aligned}
\left|a_{k}\right| & \leq 3\left(\|A \eta\|_{4}+1\right)^{2}\left(8 \max \left(\beta,\left(\|A \eta\|_{4}+8\right)^{-1}\right)\right)^{k} \\
& \leq 3\left(\|A \eta\|_{4}+1\right)^{2}(\max (8 \beta, 1))^{k}
\end{aligned}
$$

Hence, we can instead bound $\bar{F}_{\eta}$ by

$$
\bar{F}_{\eta} \leq_{(0, x, 0)} \frac{3\left(\|A \eta\|_{4}+1\right)^{2}}{1-\max \left(8+8\|A \eta\|_{\infty}, 1\right) t} .
$$

Now, we prove the geodesic has large radius of convergence. 
Lemma 81. Under the normalization $A^{T} A=I, S_{x}=I$ and Euclidean coordinate, any geodesic starting at $x$ satisfies the bound

$$
\left\|\gamma^{(k)}(0)\right\|_{2} \leq k ! c^{k}
$$

for all $k \geq 2$ where $c=512\left\|A \gamma^{\prime}(0)\right\|_{4}$.

Proof. Recall that under Euclidean coordinate, the geodesic equation is given by

$$
\gamma^{\prime \prime}=\left(A_{\gamma}^{T} A_{\gamma}\right)^{-1} A_{\gamma}^{T} s_{\gamma^{\prime}}^{2} \stackrel{\text { def }}{=} F\left(\gamma^{\prime}, \gamma\right) \text {. }
$$

So, we have that $\bar{F}_{\eta}(y, x, t)=\alpha^{2} F\left(\alpha^{-1} y+\gamma^{\prime}(0), x+\alpha t \gamma^{\prime}(0)\right)$ with $\eta=\alpha \gamma^{\prime}(0)$.

Now, we estimate $\bar{F}_{\eta}$. Lemma 80 shows that

$$
\bar{F}_{\eta} \leq_{(0, x, 0)} \frac{3\left(\|A \eta\|_{4}+1\right)^{2}}{1-\max \left(8+8\|A \eta\|_{\infty}, 1\right) t}=\frac{3\left(\alpha\left\|A \gamma^{\prime}(0)\right\|_{4}+1\right)^{2}}{1-\max \left(8+8 \alpha\left\|A \gamma^{\prime}(0)\right\|_{\infty}, 1\right) t} .
$$

Setting $\alpha=\left\|A \gamma^{\prime}(0)\right\|_{4}^{-1}$ and using $\left\|A \gamma^{\prime}(0)\right\|_{\infty} \leq\left\|A \gamma^{\prime}(0)\right\|_{4}$, we have that

$$
\bar{F}_{\eta} \leq_{(0, x, 0)} \frac{12}{1-16 t} \leq_{0} \frac{16}{1-16 t}-1-t .
$$

Lemma 78 shows that

$$
\left\|\gamma^{(k)}(0)\right\|_{2} \leq \frac{\psi^{(k)}(0)}{\alpha^{k}}
$$

for all $k \geq 2$ where $\psi(t)$ is the solution of

$$
\psi^{\prime}(t)=\frac{16}{1-16 \psi(t)} \text { with } \psi(0)=0 .
$$

Solving it, we get that

$$
\psi(t)=\frac{1}{16}(1-\sqrt{1-512 t})
$$

By Theorem 19, we have that

$$
\left|\psi^{(k)}(0)\right| \leq k !(512)^{k}
$$

Hence, we have that

$$
\left\|\gamma^{(k)}(0)\right\|_{2} \leq \frac{k !(512)^{k}}{\alpha^{k}}=k !(512)^{k}\left\|A \gamma^{\prime}(0)\right\|_{4}^{k}
$$

for all $k \geq 2$.

\subsubsection{Parallel Transport}

Motivated from the equation for parallel transport under Euclidean coordinate 4.2, we define the following auxiliary function

$$
F(t)=\left(A_{\gamma(t)}^{T} A_{\gamma(t)}\right)^{-1} A_{\gamma(t)}^{T} S_{\gamma^{\prime}(t)} A_{\gamma(t)} .
$$

The derivative bounds on parallel transport rely on the smoothness of this auxiliary function.

Lemma 82. Given a geodesic $\gamma(t)$. Under the normalization that $A^{T} A=I, S_{\gamma(0)}=I$, we have that

$$
F(t) \leq_{0} \frac{c}{2(1-2 c t)^{2}-(1-c t)^{2}} \frac{1-c t}{1-2 c t}
$$

where $F$ is defined in (6.2) and $c=512\left\|A \gamma^{\prime}(0)\right\|_{4}$. 
Proof. Lemma 81 shows that $\left\|\gamma^{(k)}(0)\right\|_{2} \leq k ! c^{k}$ for all $k \geq 2$. Therefore, we have that

$$
S_{\gamma(t)} \leq_{0} 1+t\left\|A \gamma^{\prime}(0)\right\|_{\infty}+\sum_{k \geq 2}(c t)^{k} \leq_{0} \frac{1}{1-c t} .
$$

Using Lemma 76, we have that

$$
A_{\gamma(t)} \leq_{0} \frac{1}{2-\frac{1}{1-c t}}=\frac{1-c t}{1-2 c t}
$$

and hence

$$
\left(A_{\gamma(t)}^{T} A_{\gamma(t)}\right)^{-1} A_{\gamma(t)}^{T} \leq_{0} \frac{(1-2 c t)(1-c t)}{2(1-2 c t)^{2}-(1-c t)^{2}}
$$

Now, we note that

$$
\operatorname{Diag}\left(A \gamma^{\prime}(t)\right) \leq_{0}\left\|A \gamma^{\prime}(0)\right\|_{\infty}+\sum_{k \geq 1} \frac{(k+1) ! c^{k+1} t^{k}}{k !} \leq_{0} \frac{c}{(1-c t)^{2}}
$$

and hence

$$
S_{\gamma^{\prime}}=S_{\gamma}^{-1} \operatorname{Diag}\left(A \gamma^{\prime}(t)\right) \leq_{0} \frac{1-c t}{1-2 c t} \frac{c}{(1-c t)^{2}}=\frac{c}{(1-2 c t)(1-c t)} .
$$

This gives the result.

Now, we prove parallel transport has large radius of convergence.

Lemma 83. Given a geodesic with $\gamma(0)=x$. Let $v(t)$ be the parallel transport of a unit vector along $\gamma(t)$. Under the normalization that $A^{T} A=I, S_{\gamma(0)}=I$, we have that

$$
\left\|v^{(k)}(0)\right\|_{2} \leq k !(16 c)^{k}
$$

for all $k \geq 1$ where $c=512\left\|A \gamma^{\prime}(0)\right\|_{4}$.

Proof. From (4.2), we have that

$$
\frac{d}{d t} v(t)=\left(A_{\gamma(t)}^{T} A_{\gamma(t)}\right)^{-1} A_{\gamma(t)}^{T} S_{\gamma^{\prime}(t)} A_{\gamma(t)} v(t) .
$$

Let $u(t)=v(\alpha t)$, then we have that

$$
\begin{aligned}
u^{\prime}(t) & =\alpha v^{\prime}(\alpha t) \\
& =\alpha\left(A_{\gamma(\alpha t)}^{T} A_{\gamma(\alpha t)}\right)^{-1} A_{\gamma(\alpha t)}^{T} S_{\gamma^{\prime}(\alpha t)} A_{\gamma(\alpha t)} u(t) .
\end{aligned}
$$

Let $F(x, t)=\alpha\left(A_{\gamma(\alpha t)}^{T} A_{\gamma(\alpha t)}\right)^{-1} A_{\gamma(\alpha t)}^{T} S_{\gamma^{\prime}(\alpha t)} A_{\gamma(\alpha t)} x$. Then, Lemma 82 shows that

$$
F(x, t) \leq_{(v, 0)} \frac{\alpha c}{2(1-2 \alpha c t)^{2}-(1-\alpha c t)^{2}} \frac{1-\alpha c t}{1-2 \alpha c t}(1+t) .
$$

Setting $\alpha=\frac{1}{8 c}$, we have that

$$
F(x, t) \leq_{(v, 0)} \frac{\frac{1}{8}}{2\left(1-\frac{t}{4}\right)^{2}-\left(1-\frac{t}{8}\right)^{2}} \frac{1-\frac{t}{8}}{1-\frac{t}{4}}(1+t) \leq_{0} \frac{1}{1-t} .
$$


Lemma 77 shows that

$$
\left\|u^{(k)}(0)\right\|_{2} \leq \psi^{(k)}(0)
$$

for all $k \geq 1$ where $\psi(t)$ is the solution of

$$
\psi^{\prime}(t)=\frac{1}{1-\psi(t)} \text { with } \psi(0)=0 .
$$

Solving it, we get that

$$
\psi(t)=1-\sqrt{1-2 t} .
$$

By Theorem 19, we have that for any $0 \leq t \leq \frac{1}{2}$, we have that

$$
\left\|u^{(k)}(0)\right\|_{2} \leq\left|\psi^{(k)}(0)\right| \leq k ! 2^{k}
$$

for all $k \geq 1$. For $k \geq 1$, we have that

$$
\left\|v^{(k)}(0)\right\|_{2} \leq k !(16 c)^{k}
$$

\subsubsection{Jacobi field}

Motivated from the equation for Jacobi field under orthogonal frame basis (Lemma 49), we define the following auxiliary function

$$
F(t)=X^{-1}\left(A_{\gamma}^{T} A_{\gamma}\right)^{-1}\left(A_{\gamma}^{T} S_{\gamma^{\prime}} P_{\gamma} S_{\gamma^{\prime}} A_{\gamma}-A_{\gamma}^{T} \operatorname{diag}\left(P_{\gamma} s_{\gamma^{\prime}}^{2}\right) A_{\gamma}\right) X .
$$

The derivative bounds on Jacobi field rely on the smoothness of this auxiliary function.

Lemma 84. Given a geodesic $\gamma(t)$ and an orthogonal frame $\left\{x_{i}\right\}_{i=1}^{n}$. Under the normalization that $A^{T} A=I, S_{\gamma(0)}=I$, we have that

$$
F(t) \leq_{0} \frac{12 c^{2}}{1-64 c t}
$$

where $F$ is defined in (6.4) and $c=512\left\|A \gamma^{\prime}(0)\right\|_{4}$.

Proof. We first bound the derivatives of $s_{\gamma^{\prime}}^{2}$. Using $\left\|\gamma^{(k)}(0)\right\|_{2} \leq k ! c^{k}$ for all $k \geq 2$ (Lemma 81), we have that

$$
\gamma^{\prime}(t)-\gamma^{\prime}(0) \leq_{0} \frac{d}{d t} \frac{1}{1-c t}=\frac{c}{(1-c t)^{2}} .
$$

Using $A^{T} A=I$ and $\operatorname{Diag}\left(A \gamma^{\prime}(t)\right) \leq_{0} \frac{c}{(1-c t)^{2}}$ (6.3), we have that

$$
\operatorname{Diag}\left(A \gamma^{\prime}(t)\right) A\left(\gamma^{\prime}(t)-\gamma^{\prime}(0)\right) \leq_{0}\left(\frac{c}{(1-c t)^{2}}\right)^{2} .
$$

Next, we note that

$$
\operatorname{Diag}\left(A \gamma^{\prime}(t)\right) A \gamma^{\prime}(0)=\operatorname{Diag}\left(A \gamma^{\prime}(0)\right) A\left(\gamma^{\prime}(t)-\gamma^{\prime}(0)\right)+\operatorname{Diag}\left(A \gamma^{\prime}(0)\right)^{2} .
$$

and hence

$$
\operatorname{Diag}\left(A \gamma^{\prime}(t)\right) A \gamma^{\prime}(0) \leq_{0} \frac{c^{2}}{(1-c t)^{2}}+c^{2}
$$


Combining (6.5) and (6.6), we get

$$
\operatorname{Diag}\left(A \gamma^{\prime}(t)\right) A\left(\gamma^{\prime}(t)\right) \leq_{0}\left(\frac{c}{(1-c t)^{2}}\right)^{2}+\frac{c^{2}}{(1-c t)^{2}}+c^{2} \leq_{0} \frac{3 c^{2}}{(1-c t)^{4}}
$$

In the proof of Lemma 82, we showed that $S_{\gamma} \leq_{0} \frac{1}{1-c t}$ and hence

$$
\begin{aligned}
s_{\gamma^{\prime}}^{2} & =S_{\gamma}^{-2} \operatorname{Diag}\left(A \gamma^{\prime}(t)\right) A\left(\gamma^{\prime}(t)\right) . \\
& \leq{ }_{0}\left(\frac{1}{2-\frac{1}{1-c t}}\right)^{2} \frac{3 c^{2}}{(1-c t)^{4}} \\
& =\frac{3 c^{2}}{(1-c t)^{2}(2(1-c t)-1)^{2}} .
\end{aligned}
$$

In the proof of Lemma 82, we showed that $A_{\gamma} \leq_{0} \frac{1-c t}{1-2 c t}$ and $\left(A_{\gamma}^{T} A_{\gamma}\right)^{-1} \leq_{0} \frac{(1-2 c t)^{2}}{2(1-2 c t)^{2}-(1-c t)^{2}}$. Therefore, we have that

Hence, we have

$$
P_{\gamma}=A_{\gamma}\left(A_{\gamma}^{T} A_{\gamma}\right)^{-1} A_{\gamma}^{T} \leq_{0} \frac{(1-c t)^{2}}{2(1-2 c t)^{2}-(1-c t)^{2}}
$$

$$
\begin{aligned}
P_{\gamma} s_{\gamma^{\prime}}^{2} & \leq_{0} \frac{(1-c t)^{2}}{2(1-2 c t)^{2}-(1-c t)^{2}} \frac{3 c^{2}}{(1-c t)^{2}(1-2 c t)^{2}} \\
& =\frac{3 c^{2}}{(1-2 c t)^{2}\left(2(1-2 c t)^{2}-(1-c t)^{2}\right)} .
\end{aligned}
$$

Let $Y=\left(A_{\gamma}^{T} A_{\gamma}\right)^{-1}\left(A_{\gamma}^{T} S_{\gamma^{\prime}} P_{\gamma} S_{\gamma^{\prime}} A_{\gamma}-A_{\gamma}^{T} \operatorname{diag}\left(P_{\gamma} s_{\gamma^{\prime}}^{2}\right) A_{\gamma}\right)$. By a similar proof, we have that

$$
\begin{aligned}
Y_{0} & \leq_{0} \frac{(1-2 c t)^{2}}{2(1-2 c t)^{2}-(1-c t)^{2}}\left(\frac{1-c t}{1-2 c t}\right)^{2} \\
& \left(\left(\frac{c}{(1-2 c t)(1-c t)}\right)^{2} \frac{(1-c t)^{2}}{2(1-2 c t)^{2}-(1-c t)^{2}}+\frac{3 c^{2}}{(1-2 c t)^{2}\left(2(1-2 c t)^{2}-(1-c t)^{2}\right)}\right) \\
& =\frac{4 c^{2}}{\left(2(1-2 c t)^{2}-(1-c t)^{2}\right)^{2}}\left(\frac{1-c t}{1-2 c t}\right)^{2}
\end{aligned}
$$

Next, we let $z(t)=X(t) v$ for some unit vector $v$. Since $X(t)$ is a parallel transport of $X(0)$, $z(t)$ is a parallel transport of $X(0) v$ and hence Lemma 83 shows that $\left\|z^{(k)}(t)\right\|_{2} \leq k !(16 c)^{k}$ for all $i$. For any $k$, there is unit vector $v_{k}$ such that $\left\|\frac{d}{d t^{k}} X(0)\right\|_{2}=\left\|\frac{d}{d t^{k}} X(0) v_{k}\right\|_{2}$. Hence, we have that

$$
\left\|\frac{d}{d t^{k}} X(0)\right\|_{2} \leq k !(16 c)^{k} \text {. }
$$

Therefore, we have that $X \leq_{0} \frac{1}{1-16 c t}$. Since $X(0)=I$, we have that $X^{-1} \leq_{0} \frac{1}{2-\frac{1}{1-16 c t}}=\frac{1-16 c t}{1-32 c t}$. Thus, we have that

$$
\begin{aligned}
F(t) & \leq_{0} \frac{4 c^{2}}{\left(2(1-2 c t)^{2}-(1-c t)^{2}\right)^{2}}\left(\frac{1-c t}{1-2 c t}\right)^{2} \frac{1}{1-32 c t} \\
& \leq_{0} \frac{12 c^{2}}{1-64 c t} .
\end{aligned}
$$


Now, we prove Jacobi field has large radius of convergence.

Lemma 85. Given a geodesic $\gamma(t)$ and an orthogonal frame $\left\{X_{i}\right\}_{i=1}^{n}$ along $\gamma$. Let $V(t)$ be a Jacobi field along $\gamma(t)$ with $V(0)=0$ and let $U(t)$ be the Jacobi field under the $X_{i}$ coordinates, namely, $V(t)=X(t) U(t)$. Assume that $U(0)=0$. Under the normalization that $A^{T} A=I, S_{\gamma(0)}=I$, we have that

$$
\left\|U^{(k)}(0)\right\|_{2} \leq 4(k !)(256 c)^{k-1}\left\|U^{\prime}(0)\right\|_{2}
$$

for all $k \geq 2$ where $c=512\left\|A \gamma^{\prime}(0)\right\|_{4}$.

Proof. Note that $\frac{d^{2} U(t)}{d t^{2}}+F(t) U(t)=0$ where $F(t)$ defined in (6.4). Let $\bar{F}(U, t)=\alpha^{2} F(\alpha t)\left(U+\alpha t U^{\prime}(0)\right)$ with $\alpha=\frac{1}{64 c}$.

Since the differential equation is linear, we can rescale $U$ and assume that $\left\|U^{\prime}(0)\right\|_{2}=\frac{1}{\alpha}=64 c$. that

Using $\alpha^{2} F(\alpha t) \leq_{0} \frac{12 c^{2} \alpha^{2}}{1-64 c \alpha t}$ (Lemma 84), $U \leq_{U=0} t$ and $\alpha t U^{\prime}(0) \leq_{t=0}\left\|\alpha U^{\prime}(0)\right\|_{2} t=t$, we have

$$
\begin{aligned}
\bar{F} & \leq(0,0) \\
& \leq \frac{24 c^{2} \alpha^{2} t}{1-64 c \alpha t} \\
& \leq \frac{t}{1-t} \leq_{0} \frac{2 t}{1-t}-1-t .
\end{aligned}
$$

Lemma 78 shows that that

$$
\left\|U^{(k)}(0)\right\|_{2} \leq \frac{\psi^{(k)}(0)}{\alpha^{k}}
$$

for all $k \geq 2$ where $\psi(t)$ is the solution of

$$
\psi^{\prime}(t)=\frac{2}{1-\psi(t)} \text { with } \psi(0)=0 .
$$

Solving it, we get that

$$
\psi(t)=1-\sqrt{1-4 t}
$$

By Theorem 19, we have that for any $0 \leq t \leq \frac{1}{4}$, we have that

$$
\left|\psi^{(k)}(0)\right| \leq k !(4)^{k}
$$

Hence, we have that

$$
\left\|U^{(k)}(0)\right\|_{2} \leq \frac{k ! 4^{k}}{\alpha^{k}} \leq k !(256 c)^{k}
$$

for all $k \geq 2$.

Since we have rescaled the equation, we need to rescale it back and get the result.

\subsection{Computing Geodesic Equation}

To apply Theorem 65, we define

$$
F(u, s)=A\left(A^{T} S^{-2} A\right)^{-1} A^{T} S^{-3} u^{2}
$$

The following lemma bounds the Lipschitz constant of $F$.

Lemma 86. Assuming $\frac{1}{2} \leq s_{i} \leq 2$ for all $i$. Then, we have

$$
\left\|D F(u, s)\left[d_{u}, d_{s}\right]\right\|_{2}^{2} \leq 10^{4}\left(\left\|d_{s}\right\|_{\infty}^{2}\|u\|_{4}^{4}+\left\|d_{u}\right\|_{4}^{2}\|u\|_{4}^{2}\right) .
$$


Proof. Let $A_{s}=S^{-1} A$ and $S_{d}=S^{-1} \operatorname{Diag}\left(d_{s}\right)$. Then, we have $F(u, s)=A\left(A_{s}^{T} A_{s}\right)^{-1} A_{s}^{T} S^{-2} u^{2}$. Hence, we have that

$$
\begin{aligned}
D F(u, s)\left[d_{u}, d_{s}\right]= & 2 A\left(A_{s}^{T} A_{s}\right)^{-1} A_{s}^{T} S_{d} A_{s}\left(A_{s}^{T} A_{s}\right)^{-1} A_{s}^{T} S^{-2} u^{2} \\
& -3 A\left(A_{s}^{T} A_{s}\right)^{-1} A_{s}^{T} S_{d} S^{-2} u^{2} \\
& +2 A\left(A_{s}^{T} A_{s}\right)^{-1} A_{s}^{T} S^{-2} U d_{u}
\end{aligned}
$$

Let $P=A_{s}\left(A_{s}^{T} A_{s}\right)^{-1} A_{s}^{T}$, then, we have that

$$
\begin{aligned}
\left\|D F(u, s)\left[d_{u}, d_{s}\right]\right\|_{2}^{2} \leq & 12\left(u^{2}\right)^{T} S^{-2} P S_{d} P S^{2} P S_{d} P S^{-2} u^{2} \\
& +27\left(u^{2}\right)^{T} S^{-2} S_{d} P S^{2} P S_{d} S^{-2} u^{2} \\
& +12 d_{u}^{T} S^{-2} U P S^{2} P U S^{-2} d_{u} .
\end{aligned}
$$

Using that $P \preceq I$, we have that

$$
\begin{aligned}
& \left\|D F(u, s)\left[d_{u}, d_{s}\right]\right\|_{2}^{2} \\
\leq & 12\|S\|_{\infty}^{2}\left(u^{2}\right)^{T} S^{-2} P S_{d}^{2} P S^{-2} u^{2}+27\|S\|_{\infty}^{2}\left(u^{2}\right)^{T} S^{-2} S_{d}^{2} S^{-2} u^{2}+12\|S\|_{\infty}^{2}\left\|S^{-1}\right\|_{\infty}^{4} \sum_{i}\left(d_{u}\right)_{i}^{2} u_{i}^{2} \\
\leq & 39\|S\|_{\infty}^{2}\left\|S^{-1}\right\|_{\infty}^{4}\left\|S_{d}\right\|_{\infty}^{2}\|u\|_{4}^{4}+12\|S\|_{\infty}^{2}\left\|S^{-1}\right\|_{\infty}^{4}\|u\|_{4}^{2}\left\|d_{u}\right\|_{4}^{2} \\
\leq & 39\|S\|_{\infty}^{2}\left\|S^{-1}\right\|_{\infty}^{6}\left\|d_{s}\right\|_{\infty}^{2}\|u\|_{4}^{4}+12\|S\|_{\infty}^{2}\left\|S^{-1}\right\|_{\infty}^{4}\|u\|_{4}^{2}\left\|d_{u}\right\|_{4}^{2} .
\end{aligned}
$$

Now, we use that $\frac{1}{2} \leq s_{i} \leq 2$ and get

$$
\left\|D F(u, s)\left[d_{u}, d_{s}\right]\right\|_{2}^{2} \leq 9984\left\|d_{s}\right\|_{\infty}^{2}\|u\|_{4}^{4}+768\|u\|_{4}^{2}\left\|d_{u}\right\|_{4}^{2} .
$$

Now, we can apply the collocation method to obtain a good approximation of geodesics.

Lemma 87. Let $\gamma$ be a random geodesic generated by the geodesic walk with step size $h \leq \frac{1}{10^{20} \sqrt{n}}$. With probability at least $1-O\left(\frac{1}{n}\right)$ among $\gamma$, in time $O\left(m n^{\omega-1} \log ^{2}(n / \varepsilon)\right)$, we can find $\bar{\gamma}$ such that

$$
\max _{0 \leq t \leq \ell}\|\gamma(t)-\bar{\gamma}(t)\|_{\infty} \leq \varepsilon \quad \text { and } \max _{0 \leq t \leq \ell}\left\|\gamma^{\prime}(t)-\bar{\gamma}^{\prime}\right\|_{\infty} \leq \varepsilon
$$

Furthermore, $\bar{\gamma}$ is a $O(\log (1 / \varepsilon))$ degree polynomial.

Proof. Let $s(t)=A \gamma(t)-b$. By rotating the space and rescaling the rows of $A$, we assume that $s(0)_{i}=1$ for all $i$ and $A^{T} A=I$. We define $F$ as (6.7). Then, we have that

$$
\begin{aligned}
s^{\prime \prime}(t) & =F\left(s^{\prime}, s\right), \\
s^{\prime}(0) & =A \gamma^{\prime}(0), \\
s(0) & =1 .
\end{aligned}
$$

We let $\alpha=4000 \ell$ and

$$
\begin{aligned}
K & \stackrel{\text { def }}{=} \alpha\left\|F\left(A \gamma^{\prime}(0), 1\right)\right\|_{4}+\left\|A \gamma^{\prime}(0)\right\|_{4} \\
& =\alpha\left\|A\left(A^{T} A\right)^{-1} A^{T}\left(A \gamma^{\prime}(0)\right)^{2}\right\|_{2}+\left\|A \gamma^{\prime}(0)\right\|_{4} \\
& \leq \alpha\left\|A \gamma^{\prime}(0)\right\|_{4}^{2}+\left\|A \gamma^{\prime}(0)\right\|_{4} .
\end{aligned}
$$


Using $\left\|A_{\gamma} \gamma^{\prime}(0)\right\|_{4} \leq 48 n^{-1 / 4}$ with probability $1-\frac{3}{n}$ (Lemma 53 and 54), we have that

$$
\begin{aligned}
K & \leq 4000 \ell\left(48 n^{-1 / 4}\right)^{2}+48 n^{-1 / 4} \leq 100 n^{-1 / 4}, \\
\alpha K & \leq 4000 \ell \cdot 100 n^{-1 / 4} \leq \frac{1}{2}
\end{aligned}
$$

For any $\left\|u-s^{\prime}(0)\right\|_{4} \leq K \leq 100 n^{-1 / 4},\|s-1\|_{4} \leq \alpha K \leq \frac{1}{2}$, Lemma 86 shows that

$$
\begin{aligned}
\left\|D F(u, s)\left[d_{u}, d_{s}\right]\right\|_{4} & \leq\left\|D F(u, s)\left[d_{u}, d_{s}\right]\right\|_{2} \\
& \leq 10^{2}\left(\left\|d_{s}\right\|_{\infty}\|u\|_{4}^{2}+\left\|d_{u}\right\|_{4}\|u\|_{4}\right) \\
& \leq 10^{7}\left(n^{-1 / 2}\left\|d_{s}\right\|_{\infty}+n^{-1 / 4}\left\|d_{u}\right\|_{4}\right) .
\end{aligned}
$$

Therefore, for any $\left\|u_{1}-s^{\prime}(0)\right\|_{4} \leq K,\left\|s_{1}-1\right\|_{4} \leq \alpha K,\left\|u_{2}-s^{\prime}(0)\right\|_{4} \leq K,\left\|s_{2}-1\right\|_{4} \leq \alpha K$, we have that

$$
\begin{aligned}
\left\|F\left(u_{1}, s_{1}\right)-F\left(u_{2}, s_{2}\right)\right\|_{4} & \leq 10^{7} n^{-1 / 4}\left\|u_{1}-u_{2}\right\|_{4}+10^{7} n^{-1 / 2}\left\|s_{1}-s_{2}\right\|_{4} \\
& \leq \frac{1}{\alpha}\left\|u_{1}-u_{2}\right\|_{4}+\frac{1}{\alpha^{2}}\left\|s_{1}-s_{2}\right\|_{4} .
\end{aligned}
$$

Since $\gamma$ is analytic and $\left\|\gamma^{(k)}(0)\right\|_{2}=O\left(k ! n^{-k / 4}\right)$ (Lemma 83), $\gamma(t)$ is $\varepsilon$ close to the following polynomial

$$
\sum_{k=0}^{\Theta(\log (1 / \varepsilon))} \frac{1}{k !} \gamma^{(k)}(0) t^{k}
$$

for $0 \leq t \leq c n^{1 / 4}$ for some small constant $c$. Hence, we can apply Theorem 69 and find $\bar{\gamma}$ such that $\|\gamma-\bar{\gamma}\|_{4} \leq \varepsilon$ and $\left\|\gamma^{\prime}-\bar{\gamma}^{\prime}\right\|_{4} \leq \varepsilon$ in $O\left(n \log ^{3}(n K / \varepsilon)\right)$ time plus $O\left(\log ^{2}(K / \varepsilon)\right)$ evaluations of $F$. Note that each evaluation of $F$ involves solving a linear system and hence it takes $O\left(m n^{\omega-1}\right)$. Therefore, the total running time is $O\left(m n^{\omega-1} \log ^{2}(n / \varepsilon)\right)$.

\subsection{Computing Parallel Transport}

Lemma 88. Given $\gamma$ be a random geodesic generated by the geodesic walk with step size $h \leq$ $\frac{1}{10^{20} \sqrt{n}}$ and an unit vector $v$. Let $v(t)$ be the parallel transport of a unit vector along $\gamma(t)$. With probability at least $1-O\left(\frac{1}{n}\right)$ among $\gamma$, in time $O\left(m n^{\omega-1} \log ^{2}(n / \varepsilon)\right)$, we can find $\bar{v}$ such that $\max _{0 \leq t \leq \ell}\|v(t)-\bar{v}(t)\|_{\infty} \leq \varepsilon$. Furthermore, $\bar{v}$ is a $O(\log (1 / \varepsilon))$ degree polynomial.

Similarly, given a basis $\left\{v_{i}\right\}_{i=1}^{n}$, with probability at least $1-O\left(\frac{1}{n}\right)$, in time $O\left(m n^{\omega-1} \log ^{2}(n / \varepsilon)\right)$, we can find an approximate parallel transport $\overline{v_{i}}(t)$ of $\left\{v_{i}\right\}_{i=1}^{n}$ along $\gamma(t)$ such that $\max _{0 \leq t \leq \ell}\left\|v_{i}(t)-\bar{v}_{i}(t)\right\|_{\infty} \leq$ $\varepsilon$ for all $i$.

Proof. Recall that the equation for parallel transport (4.2) is given by

$$
\frac{d}{d t} v(t)=\left(A_{\gamma(t)}^{T} A_{\gamma(t)}\right)^{-1} A_{\gamma(t)}^{T} S_{\gamma^{\prime}(t)} A_{\gamma(t)} v
$$

By rotating the space and rescaling the rows of $A$, we assume that $s(\gamma(0))_{i}=1$ for all $i$ and $A^{T} A=I$. In the proof of Lemma 87, we know that $\frac{1}{2} \leq s(\gamma(t))_{i} \leq 2$ for all $0 \leq t \leq \ell$. For any unit 
vector $u$, we have

$$
\begin{aligned}
\left\|\left(A_{\gamma}^{T} A_{\gamma}\right)^{-1} A_{\gamma}^{T} S_{\gamma^{\prime}} A_{\gamma} u\right\|_{2} & \leq 2\left\|\left(A_{\gamma}^{T} A_{\gamma}\right)^{-1 / 2} A_{\gamma}^{T} S_{\gamma^{\prime}} A_{\gamma} u\right\|_{2} \\
& \leq 2\left\|S_{\gamma^{\prime}}\right\|_{\infty}\left\|A_{\gamma} u\right\|_{2} \\
& \leq 4\left\|S_{\gamma^{\prime}}\right\|_{\infty} \leq 192\left(\sqrt{\frac{\log n}{n}}+\sqrt{h}\right)
\end{aligned}
$$

where we used Lemma 54 in the last line. Using $h \leq \frac{1}{10^{20} \sqrt{n}}$, we have that

$$
\left\|\left(A_{\gamma}^{T} A_{\gamma}\right)^{-1} A_{\gamma}^{T} S_{\gamma^{\prime}} A_{\gamma}\right\|_{2} \leq \frac{1}{2000 \ell} .
$$

Since $v$ is analytic and $\left\|v^{(k)}(0)\right\|_{2}=O\left(k ! n^{-k / 4}\right)$ (Lemma 83), $v(t)$ is $\varepsilon$ close to a polynomial with degree $O(\log (1 / \varepsilon))$ for $0 \leq t \leq c n^{1 / 4}$ for some small constant $c$. Hence, we can apply Theorem 65 and find $\bar{v}$ such that $\|v-\bar{v}\|_{2} \leq \varepsilon$ in $O\left(n \log ^{3}(n / \varepsilon)\right)$ time plus $O\left(\log ^{2}(1 / \varepsilon)\right)$ evaluations of $F$. Note that each evaluation of $F$ involves solving a linear system and hence it takes $O\left(m n^{\omega-1}\right)$. Therefore, the total running time is $O\left(m n^{\omega-1} \log ^{2}(n / \varepsilon)\right)$.

For the last result, we note that each evaluation of $F$ becomes computing matrix inverse and performing matrix multiplication and they can be done in again $O\left(m n^{\omega-1}\right)$ time.

\subsection{Computing Jacobi field}

Lemma 89. Given $\gamma$ be a random geodesic generated by the geodesic walk with step size $h \leq \frac{1}{10^{20} \sqrt{n}}$. Let $\left\{X_{i}\right\}_{i=1}^{n}$ be an orthogonal frame along $\gamma$. Let $v(t)$ be a Jacobi field along $\gamma(t)$ with $v(0)=0$ and $\left\|v^{\prime}(0)\right\| \leq \cdots$. Let $u(t)$ be the Jacobi field under the $X_{i}$ coordinates, namely, $v(t)=X(t) u(t)$. With probability at least $1-O\left(\frac{1}{n}\right)$ among $\gamma$, In time $O\left(m n^{\omega-1} \log ^{2}(n / \varepsilon)\right)$, we can find $\bar{u}$ such that $\max _{0 \leq t \leq \ell}\|u(t)-\bar{u}(t)\|_{\infty} \leq \varepsilon$. Furthermore, $\bar{u}$ is a $O(\log (1 / \varepsilon))$ degree polynomial.

Proof. Recall that the equation for Jacobi field 49 is given by

$$
\frac{d^{2} u}{d t^{2}}+X^{-1}\left(A_{\gamma}^{T} A_{\gamma}\right)^{-1}\left(A_{\gamma}^{T} S_{\gamma^{\prime}} P_{\gamma} S_{\gamma^{\prime}} A_{\gamma}-A_{\gamma}^{T} \operatorname{Diag}\left(P_{\gamma} s_{\gamma^{\prime}}^{2}\right) A_{\gamma}\right) X u=0 .
$$

By rotating the space and rescaling the rows of $A$, we assume that $s(\gamma(0))_{i}=1$ for all $i$ and $A^{T} A=I$. In the proof of Lemma 87, we know that $\frac{1}{2} \leq s(\gamma(t))_{i} \leq 2$ for all $0 \leq t \leq \ell$. Since $X$ is an orthogonal frame, we have $X X^{T}=X X^{T}=I$. Hence, for any unit vector $v$, we have

$$
\begin{aligned}
& \left\|X^{-1}\left(A_{\gamma}^{T} A_{\gamma}\right)^{-1}\left(A_{\gamma}^{T} S_{\gamma^{\prime}} P_{\gamma} S_{\gamma^{\prime}} A_{\gamma}-A_{\gamma}^{T} \operatorname{Diag}\left(P_{\gamma} s_{\gamma^{\prime}}^{2}\right) A_{\gamma}\right) X v\right\|_{2} \\
\leq & 2\left\|\left(A_{\gamma}^{T} A_{\gamma}\right)^{-1 / 2}\left(A_{\gamma}^{T} S_{\gamma^{\prime}} P_{\gamma} S_{\gamma^{\prime}} A_{\gamma}-A_{\gamma}^{T} \operatorname{Diag}\left(P_{\gamma} s_{\gamma^{\prime}}^{2}\right) A_{\gamma}\right) X v\right\|_{2} \\
\leq & 2\left\|S_{\gamma^{\prime}} P_{\gamma} S_{\gamma^{\prime}} A_{\gamma} X v\right\|_{2}+2\left\|\operatorname{Diag}\left(P_{\gamma^{\prime}} s_{\gamma^{\prime}}^{2}\right) A_{\gamma} X v\right\|_{2} .
\end{aligned}
$$

Using $\left\|S_{\gamma^{\prime}}\right\|_{2} \leq 48\left(\sqrt{\frac{\log n}{n}}+\sqrt{h}\right)$ and $\left\|\operatorname{Diag}\left(P_{\gamma} s_{\gamma^{\prime}}^{2}\right)\right\|_{2} \leq\left\|P_{\gamma} s_{\gamma^{\prime}}^{2}\right\|_{2} \leq\left\|s_{\gamma^{\prime}}\right\|_{4}^{2} \leq 10^{4} n^{-1 / 2}$ (Lemma 54), we have that

$$
\begin{aligned}
& \left\|X^{-1}\left(A_{\gamma}^{T} A_{\gamma}\right)^{-1}\left(A_{\gamma}^{T} S_{\gamma^{\prime}} P_{\gamma} S_{\gamma^{\prime}} A_{\gamma}-A_{\gamma}^{T} \operatorname{Diag}\left(P_{\gamma} s_{\gamma^{\prime}}^{2}\right) A_{\gamma}\right) X v\right\|_{2} \\
\leq & 2\left(48\left(\sqrt{\frac{\log n}{n}}+\sqrt{h}\right)\right)^{2}\left\|A_{\gamma} X v\right\|_{2}+4 \cdot 10^{4} n^{-1 / 2}\left\|A_{\gamma} X v\right\|_{2} \\
\leq & \frac{1}{(4000 \ell)^{2}}
\end{aligned}
$$


where we used $h \leq \frac{1}{10^{20} \sqrt{n}}$ in the last line.

Since $u$ is analytic and $\left\|u^{(k)}(0)\right\|_{2}=O\left(k ! n^{-k / 4}\right)$ (Lemma 85), $u(t)$ is $\varepsilon$ close to a polynomial with degree $O(\log (1 / \varepsilon))$ for $0 \leq t \leq c n^{1 / 4}$ for some small constant $c$. Hence, we can apply Theorem 65 and find $\bar{u}$ such that $\|u-\bar{u}\|_{2} \leq \varepsilon$ in $O\left(n \log ^{3}(n / \varepsilon)\right)$ time plus $O\left(\log ^{2}(1 / \varepsilon)\right)$ evaluations of $F$. Note that each evaluation of $F$ involves computing matrix inversions and matrix multiplications and hence it takes $O\left(m n^{\omega-1}\right)$. Therefore, the total running time is $O\left(m n^{\omega-1} \log ^{2}(n / \varepsilon)\right)$.

\subsection{Computing Geodesic Walk}

Proof of Theorem 46. To implement the geodesic walk, we use Lemma 87 to compute the geodesic, Lemma 88 to compute an orthogonal frame along the geodesic and Lemma 89 to compute the Jacobi field along. Using the Jacobi field, we can use (3.2) and Lemma 34 to compute the probability from $x$ to $y$ and the probability from $y$ to $x$. Using these probabilities, we can implement the rejection sampling. It suffices to compute the geodesic and the probability up to $1 / n^{O(1)}$ accuracy and hence these operations can be done in time $O\left(m n^{\omega-1} \log ^{2}(n)\right)$.

Note that we only use randomness to prove that $V(\gamma)$ is small (Lemma 53) and they can be checked. When we condition our walk to that, we only change the distribution by very small amount. Hence, this result is stated without mentioning the success probability.

\section{References}

[1] Sébastien Bubeck and Ronen Eldan. The entropic barrier: a simple and optimal universal self-concordant barrier. arXiv preprint arXiv:1412.1587, 2014.

[2] Sébastien Bubeck, Ronen Eldan, and Joseph Lehec. Sampling from a log-concave distribution with projected langevin Monte Carlo. arXiv preprint arXiv:1507.02564, 2015.

[3] B. Cousins and S. Vempala. A cubic algorithm for computing Gaussian volume. In SODA, pages 1215-1228, 2014.

[4] Arnak S Dalalyan. Theoretical guarantees for approximate sampling from smooth and logconcave densities. arXiv preprint arXiv:1412.7392, 2014.

[5] A. B. Dieker. Reflected Brownian Motion. John Wiley \& Sons, Inc., 2010.

[6] A Dutt, M Gu, and V Rokhlin. Fast algorithms for polynomial interpolation, integration, and differentiation. SIAM Journal on Numerical Analysis, 33(5):1689-1711, 1996.

[7] M. E. Dyer and A. M. Frieze. Computing the volume of a convex body: a case where randomness provably helps. In Proc. of AMS Symposium on Probabilistic Combinatorics and Its Applications, pages 123-170, 1991.

[8] M. E. Dyer, A. M. Frieze, and R. Kannan. A random polynomial time algorithm for approximating the volume of convex bodies. In STOC, pages 375-381, 1989.

[9] M. E. Dyer, A. M. Frieze, and R. Kannan. A random polynomial-time algorithm for approximating the volume of convex bodies. J. ACM, 38(1):1-17, 1991.

[10] J. Michael Harrison. Brownian motion and stochastic flow systems. Wiley series in probability and mathematical statistics. Wiley, New York, 1985. 
[11] Roland Hildebrand. Canonical barriers on convex cones. Mathematics of operations research, 39(3):841-850, 2014.

[12] Arieh Iserles. A first course in the numerical analysis of differential equations. Number 44. Cambridge university press, 2009.

[13] Jürgen Jost. Riemannian geometry and geometric analysis. Springer Science \& Business Media, 2008.

[14] R. Kannan, L. Lovász, and M. Simonovits. Random walks and an $O^{*}\left(n^{5}\right)$ volume algorithm for convex bodies. Random Structures and Algorithms, 11:1-50, 1997.

[15] R. Kannan and H. Narayanan. Random walks on polytopes and an affine interior point method for linear programming. In STOC, pages 561-570, 2009.

[16] Narendra Karmarkar. Riemannian geometry underlying interior-point methods for linear programming. Contemporary Mathematics, 114:51-75, 1990.

[17] Yin Tat Lee and Aaron Sidford. Path finding methods for linear programming: Solving linear programs in o (vrank) iterations and faster algorithms for maximum flow. In Foundations of Computer Science (FOCS), 2014 IEEE 55 th Annual Symposium on, pages 424-433. IEEE, 2014.

[18] Yin Tat Lee and Aaron Sidford. Efficient inverse maintenance and faster algorithms for linear programming. In Foundations of Computer Science (FOCS), 2015 IEEE $56^{\text {th }}$ Annual Symposium on, pages 230-249. IEEE, 2015.

[19] Yin Tat Lee, Aaron Sidford, and Sam Chiu-wai Wong. A faster cutting plane method and its implications for combinatorial and convex optimization. In Foundations of Computer Science (FOCS), 2015 IEEE 56th Annual Symposium on, pages 1049-1065. IEEE, 2015.

[20] L. Lovász. How to compute the volume? Jber. d. Dt. Math.-Verein, Jubiläumstagung 1990, pages 138-151, 1990.

[21] L. Lovász. Hit-and-run mixes fast. Math. Prog., 86:443-461, 1998.

[22] L. Lovász and M. Simonovits. Mixing rate of Markov chains, an isoperimetric inequality, and computing the volume. In ROCS, pages 482-491, 1990.

[23] L. Lovász and M. Simonovits. On the randomized complexity of volume and diameter. In Proc. $33^{\text {rd }}$ IEEE Annual Symp. on Found. of Comp. Sci., pages 482-491, 1992.

[24] L. Lovász and M. Simonovits. Random walks in a convex body and an improved volume algorithm. In Random Structures and Alg., volume 4, pages 359-412, 1993.

[25] L. Lovász and S. Vempala. Fast algorithms for logconcave functions: sampling, rounding, integration and optimization. In FOCS, pages 57-68, 2006.

[26] L. Lovász and S. Vempala. Hit-and-run from a corner. SIAM J. Computing, 35:985-1005, 2006.

[27] Oren Mangoubi and Aaron Smith. Rapid mixing of geodesic walks on manifolds with positive curvature. arXiv preprint arXiv:1609.02901, 2016. 
[28] Hariharan Narayanan. Randomized interior point methods for sampling and optimization. Annals of Applied Probability, 26(1):597-641, February 2016.

[29] Yurii Nesterov and Arkadi Nemirovski. Primal central paths and riemannian distances for convex sets. Foundations of Computational Mathematics, 8(5):533-560, 2008.

[30] Yurii Nesterov, Arkadii Nemirovskii, and Yinyu Ye. Interior-point polynomial algorithms in convex programming, volume 13. SIAM, 1994.

[31] Yurii E Nesterov, Michael J Todd, et al. On the riemannian geometry defined by self-concordant barriers and interior-point methods. Foundations of Computational Mathematics, 2(4):333-361, 2002.

[32] Yann Ollivier. A visual introduction to riemannian curvatures and some discrete generalizations. Analysis and Geometry of Metric Measure Spaces: Lecture Notes of the 50th Séminaire de Mathématiques Supérieures (SMS), Montréal, 2011, pages 197-219, 2013.

[33] R.L. Smith. Efficient Monte-Carlo procedures for generating points uniformly distributed over bounded regions. Operations Res., 32:1296-1308, 1984.

[34] Damian Straszak and Nisheeth K Vishnoi. On a natural dynamics for linear programming. arXiv preprint arXiv:1511.07020, 2015.

[35] Burt Totaro. The curvature of a hessian metric. International Journal of Mathematics, 15(04):369-391, 2004.

[36] S. Vempala. Geometric random walks: A survey. MSRI Combinatorial and Computational Geometry, 52:573-612, 2005. 


\section{A Additional proofs}

Proof of Lemma 13. Let $T(y)=\exp _{x}(y)$. Then, we have

$$
F(T(y))=y \text {. }
$$

Therefore,

$$
D F(T(y))[D T(y)[h]]=h
$$

and

$$
D^{2} F(T(y))[D T(y)[h], D T(y)[h]]+D F(T(y))\left[D^{2} T(y)[h, h]\right]=0 .
$$

By the geodesic equation, we have

$$
\frac{d^{2} x_{k}}{d t^{2}}+\sum_{i, j} \Gamma_{i j}^{k} \frac{d x_{i}}{d t} \frac{d x_{j}}{d t}=0
$$

Therefore,

$$
T(y)=x+y-\frac{1}{2} \sum_{i, j} \Gamma_{i j}^{k} y_{i} y_{j}+O\left(\|y\|^{3}\right) .
$$

Putting $y=0$, we have $D T(0)[h]=h$ and hence $D F(x)[h]=h$. Now, we note that

$$
D^{2} F(x)[D T(0)[h], D T(0)[h]]+D F(x)\left[D^{2} T(0)[h, h]\right]=0 .
$$

Hence,

$$
D^{2} F(x)[h, h]=-D^{2} T(0)[h, h] .
$$

Using (A.1), we have $D^{2} T_{k}(0)[h, h]=-h^{T} \Gamma^{k} h$ and hence $D^{2} F_{k}(x)[h, h]=h^{T} \Gamma^{k} h$.

Lemma 90. For $p \geq 1$, we have

$$
P_{x \sim N(0, I)}\left(\|A x\|_{p}^{p} \leq\left(\left(\frac{2^{p / 2} \Gamma\left(\frac{p+1}{2}\right)}{\sqrt{\pi}} \sum_{i}\left\|a_{i}\right\|_{2}^{p}\right)^{1 / p}+\|A\|_{2 \rightarrow p} t\right)^{p}\right) \leq 1-\exp \left(-\frac{t^{2}}{2}\right) .
$$

In particular, we have

$$
P_{x \sim N(0, I)}\left(\|A x\|_{4}^{4} \leq\left(\left(3 \sum_{i}\left\|a_{i}\right\|_{2}^{4}\right)^{1 / 4}+\|A\|_{2 \rightarrow 4} t\right)^{4}\right) \leq 1-\exp \left(-\frac{t^{2}}{2}\right) .
$$

Proof. Let $F(x)=\|A x\|_{p}$. Since $|F(x)-F(y)| \leq\|A\|_{2 \rightarrow p}\|x-y\|_{2}$, Gaussian concentration shows that

$$
P_{x \sim N(0, I)}\left(F(x) \leq \mathbb{E} F(x)+\|A\|_{2 \rightarrow p} t\right) \leq 1-\exp \left(-\frac{t^{2}}{2}\right) .
$$

Since $x^{p}$ is convex, we have that

$$
\begin{aligned}
\mathbb{E}\|A x\|_{p} & \leq\left(\mathbb{E}\|A x\|_{p}^{p}\right)^{1 / p}=\left(\sum_{i} \mathbb{E}\left|a_{i}^{T} x\right|^{p}\right)^{1 / p} \\
& =\left(\mathbb{E}_{t \sim N(0,1)}|t|^{p} \sum_{i}\left\|a_{i}\right\|_{2}^{p}\right)^{1 / p} \\
& =\left(\frac{2^{p / 2} \Gamma\left(\frac{p+1}{2}\right)}{\sqrt{\pi}} \sum_{i}\left\|a_{i}\right\|_{2}^{p}\right)^{1 / p} .
\end{aligned}
$$


Lemma 91. Let $\psi(x)=\frac{\sqrt{1-y^{2}} \cos \left(d \cos ^{-1} x\right)}{d(x-y)}$ where $y=\cos \left(\frac{2 k-1}{2 d} \pi\right)$ for some integer $k \in[d]$. Then, we have that

$$
\left|\int_{-1}^{t} \psi(x) d x\right| \leq \frac{2000}{d}
$$

for any $-1 \leq t \leq 1$.

Proof. For $d=1$, we have that $y=0$ and $\psi(x)=1$. Hence, we have the result.

From now on, we assume $d \geq 2$. Let $z_{j}=\cos (j \pi / d)$. Given $j \in[d]$ with $j \neq k$. Let

$$
\bar{j}=\left\{\begin{array}{ll}
j & \text { if }\left|z_{j}-y\right| \leq\left|z_{j-1}-y\right| \\
j-1 & \text { otherwises }
\end{array} .\right.
$$

Then, we have that

$$
\begin{aligned}
\left|\int_{z_{j-1}}^{z_{j}} \psi(x) d x\right| & \leq \frac{\sqrt{1-y^{2}}}{d}\left|\int_{z_{j-1}}^{z_{j}} \frac{\cos \left(d \cos ^{-1} x\right)}{z_{\bar{j}}-y} d x\right|+\frac{\sqrt{1-y^{2}}}{d} \int_{z_{j-1}}^{z_{j}}\left|\frac{\cos \left(d \cos ^{-1} x\right)\left(z_{\bar{j}}-x\right)}{\left(z_{\bar{j}}-y\right)(x-y)}\right| d x \\
& \leq \frac{\sqrt{1-y^{2}}}{d}\left|\int_{z_{j-1}}^{z_{j}} \frac{\cos \left(d \cos ^{-1} x\right)}{z_{\bar{j}}-y} d x\right|+\frac{\sqrt{1-y^{2}}}{d} \frac{\left(z_{j}-z_{j-1}\right)^{2}}{\left(z_{\bar{j}}-y\right)^{2}} \\
& =\frac{\sqrt{1-y^{2}}}{d\left(d^{2}-1\right)}\left|\frac{z_{j}-z_{j-1}}{z_{\bar{j}}-z_{k-\frac{1}{2}}}\right|+\frac{\sqrt{1-y^{2}}}{d} \frac{\left(z_{j}-z_{j-1}\right)^{2}}{\left(z_{\bar{j}}-z_{k-\frac{1}{2}}\right)^{2}}
\end{aligned}
$$

Now, we upper bound the term $\left(z_{j}-z_{j-1}\right) /\left(z_{\bar{j}}-z_{k-\frac{1}{2}}\right)$. By trigonometric formulas, we have

$$
\left|\frac{z_{j}-z_{j-1}}{z_{\bar{j}}-z_{k-\frac{1}{2}}}\right|=\left|\frac{\cos (j \pi / d)-\cos ((j-1) \pi / d)}{\cos (\bar{j} \pi / d)-\cos \left(\left(k-\frac{1}{2}\right) \pi / d\right)}\right|=\left|\frac{\sin \left(\frac{\pi}{2 d}\right) \sin \left(\left(j-\frac{1}{2}\right) \frac{\pi}{d}\right)}{\sin \left(\left(\frac{\bar{j}-k+\frac{1}{2}}{2}\right) \frac{\pi}{d}\right) \sin \left(\left(\frac{\bar{j}+k-\frac{1}{2}}{2}\right) \frac{\pi}{d}\right)}\right| .
$$

Note that $\left|\left(\bar{j}-k+\frac{1}{2}\right) \pi /(2 d)\right| \leq \frac{\pi}{2}$ and hence

$$
\left|\sin \left(\frac{\bar{j}-k+\frac{1}{2}}{2} \frac{\pi}{d}\right)\right| \geq \frac{2}{\pi}\left|\frac{\bar{j}-k+\frac{1}{2}}{2} \frac{\pi}{d}\right|=\left|\frac{\bar{j}-k+\frac{1}{2}}{d}\right| .
$$

By symmetric, we can assume $k-\frac{1}{2} \leq \frac{d}{2}$ and hence $\left|\left(\bar{j}-k+\frac{1}{2}\right) \pi /(2 d)\right| \leq \frac{3 \pi}{4}$ and

$$
\left|\sin \left(\frac{\bar{j}+k-\frac{1}{2}}{2} \frac{\pi}{d}\right)\right| \geq \frac{4}{3 \sqrt{2} \pi}\left|\frac{\bar{j}+k-\frac{1}{2}}{2} \frac{\pi}{d}\right|=\frac{\sqrt{2}}{3}\left|\frac{\bar{j}+k-\frac{1}{2}}{d}\right| .
$$

Therefore, we have that

$$
\left|\frac{z_{j}-z_{j-1}}{z_{\bar{j}}-z_{k-\frac{1}{2}}}\right| \leq \frac{\frac{\pi}{2 d}\left|\left(j-\frac{1}{2}\right) \frac{\pi}{d}\right|}{\left|\frac{\bar{j}-k+\frac{1}{2}}{d}\right| \frac{\sqrt{2}}{3}\left|\frac{\bar{j}+k-\frac{1}{2}}{d}\right|}=\frac{3 \pi^{2}}{2 \sqrt{2}} \frac{\left|j-\frac{1}{2}\right|}{\left|\bar{j}-k+\frac{1}{2}\right|\left|\bar{j}+k-\frac{1}{2}\right|} .
$$

Note that $\left|\bar{j}+k-\frac{1}{2}\right| \geq j-\frac{1}{2} \geq 0$ and hence

$$
\left|\frac{z_{j}-z_{j-1}}{z_{\bar{j}}-z_{k-\frac{1}{2}}}\right| \leq \frac{3 \pi^{2}}{2 \sqrt{2}} \frac{1}{\left|\bar{j}-k+\frac{1}{2}\right|} .
$$


Putting it into (A.2), we get

$$
\begin{aligned}
\left|\int_{z_{j-1}}^{z_{j}} \psi(x) d x\right| & \leq \frac{1}{d\left(d^{2}-1\right)} \frac{3 \pi^{2}}{2 \sqrt{2}} \frac{1}{\left|\bar{j}-k+\frac{1}{2}\right|}+\frac{1}{d}\left(\frac{3 \pi^{2}}{2 \sqrt{2}} \frac{1}{\left|\bar{j}-k+\frac{1}{2}\right|}\right)^{2} \\
& \leq \frac{21}{d\left(d^{2}-1\right)}+\frac{110}{d} \frac{1}{\left|\bar{j}-k+\frac{1}{2}\right|^{2}} .
\end{aligned}
$$

Using $d \geq 2$, we have

$$
\begin{aligned}
\left|\int_{-1}^{t} \psi(x) d x\right| & \leq \sum_{k \neq j}\left|\int_{z_{j-1}}^{z_{j}} \psi(x) d x\right|+\int_{z_{k-1}}^{z_{k}}|\psi(x)| d x \\
& \leq \frac{21}{d^{2}-1}+\frac{220}{d}\left(\frac{1}{0.5^{2}}+\frac{1}{1.5^{2}}+\frac{1}{2.5^{2}}+\cdots\right)+\int_{z_{k-1}}^{z_{k}}|\psi(x)| d x \\
& \leq \frac{1200}{d}+\int_{z_{k-1}}^{z_{k}}|\psi(x)| d x
\end{aligned}
$$

To bound $\psi$ over $\left[z_{k}, z_{k-1}\right]$, we write $x=\cos \left(\frac{2 k-1-\theta}{2 d} \pi\right)$ with $-1 \leq \theta \leq 1$. We have that

$$
\begin{aligned}
\psi(x) & =\frac{\sqrt{1-\cos \left(\frac{2 k-1}{2 d} \pi\right)^{2}} \cos \left(d \cos ^{-1} \cos \left(\frac{2 k-1-\theta}{2 d} \pi\right)\right)}{d\left(\cos \left(\frac{2 k-1-\theta}{2 d} \pi\right)-\cos \left(\frac{2 k-1}{2 d} \pi\right)\right)} \\
& =\frac{-\sin \left(\frac{2 k-1}{2 d} \pi\right) \sin \left(\frac{\theta}{2} \pi\right)}{2 d\left(\sin \left(\frac{\theta}{2 d} \pi\right) \sin \left(\frac{2 k-1-\theta / 2}{2 d} \pi\right)\right)} .
\end{aligned}
$$

Since $-1 \leq \theta \leq 1$ and $d \geq 2$, we have

$$
\begin{aligned}
|\psi(x)| & \leq \frac{\sin \left(\frac{2 k-1}{2 d} \pi\right)\left|\frac{\theta}{2} \pi\right|}{2 d \frac{\sqrt{2}|\theta|}{d} \sin \left(\frac{2 k-1-\theta / 2}{2 d} \pi\right)}=\frac{\pi}{4 \sqrt{2}} \frac{\sin \left(\frac{2 k-1}{2 d} \pi\right)}{\sin \left(\frac{2 k-1-\theta / 2}{2 d} \pi\right)} \\
& \leq \frac{\pi}{4 \sqrt{2}} \frac{\sin \left(\frac{1}{2 d} \pi\right)}{\sin \left(\frac{1}{4 d} \pi\right)} \leq 2 .
\end{aligned}
$$

Putting it into (A.3), we get

$$
\left|\int_{-1}^{t} \psi(x) d x\right| \leq \frac{1200}{d}+2\left|z_{k}-z_{k-1}\right| \leq \frac{2000}{d} .
$$

Exercise 92. Prove Fact 3 for Hessian manifolds using Lemma 10 as a definition.

Proof. We ignore the proof for (1) and (7) since we use Lemma 10 as the definition here. (3) and (4) are immediate from the definition. 
(5) By the definition of $D_{t}$, we have that $D_{t}=\nabla_{c^{\prime}}$. Using the definition of $\nabla_{v} u$, we have

$$
\begin{aligned}
& \left\langle D_{t} u, w\right\rangle_{c(t)}+\left\langle u, D_{t} w\right\rangle_{c(t)} \\
= & \left\langle\sum_{i k} c_{i}^{\prime} \frac{\partial u_{k}}{\partial x_{i}} e_{k}+\sum_{i j k} c_{i}^{\prime} u_{j} \Gamma_{i j}^{k} e_{k}, w\right\rangle_{c(t)}+\left\langle u, \sum_{i k} c_{i}^{\prime} \frac{\partial w_{k}}{\partial x_{i}} e_{k}+\sum_{i j k} c_{i}^{\prime} w_{j} \Gamma_{i j}^{k} e_{k}\right\rangle_{c(t)} \\
= & \left\langle\frac{d u(c(t))}{d t}, w(c(t))\right\rangle_{c(t)}+\left\langle u(c(t)), \frac{d w(c(t))}{d t}\right\rangle_{c(t)} \\
& +\frac{1}{2}\left\langle\sum_{i j k l} \gamma_{i}^{\prime} u_{j} g^{k l} \phi_{i j l} e_{k}, w\right\rangle_{c(t)}+\frac{1}{2}\left\langle u, \sum_{i j k l} \gamma_{i}^{\prime} w_{j} g^{k l} \phi_{i j l} e_{k}\right\rangle_{c(t)} \\
\triangleq & \left\langle\frac{d u(c(t))}{d t}, w(c(t))\right\rangle_{c(t)}+\left\langle u(c(t)), \frac{d w(c(t))}{d t}\right\rangle_{c(t)} \\
& +\frac{1}{2} \sum_{i j k l p} \gamma_{i}^{\prime} u_{j} g^{k l} \phi_{i j l} g_{k p} w_{p}+\frac{1}{2} \sum_{i j k l p} \gamma_{i}^{\prime} w_{j} g^{k l} \phi_{i j l} g_{k p} u_{p} \\
\text { 中p } & \left\langle\frac{d u(c(t))}{d t}, w(c(t))\right\rangle_{c(t)}+\left\langle u(c(t)), \frac{d w(c(t))}{d t}\right\rangle_{c(t)} \\
& +\frac{1}{2} \sum_{i j k l p} \gamma_{i}^{\prime} u_{j} \phi_{i j p} w_{p}+\frac{1}{2} \sum_{i j k l p} \gamma_{i}^{\prime} w_{j} \phi_{i j p} u_{p} \\
= & \left\langle\frac{d u(c(t))}{d t}, w(c(t))\right\rangle_{c(t)}+\left\langle u(c(t)), \frac{d w(c(t))}{d t}\right\rangle_{c(t)}+u(t)^{T} \frac{d}{d t} g(\gamma(t)) w(t) \\
= & \frac{d}{d t}\langle u, w\rangle_{c(t)}
\end{aligned}
$$

where we used $\langle a, b\rangle_{c(t)}=\sum a_{i} g_{i j} b_{j}$ on $\diamond$ and $g^{k l}$ is the inverse of $g_{k p}$ on

(6) For any map $c(t, s)$ on $M$, we have that

$$
\begin{aligned}
D_{s} \frac{\partial c}{\partial t} & =\frac{\partial}{\partial s} \frac{\partial c}{\partial t}+\sum_{i j k} \frac{\partial c_{i}}{\partial s} \frac{\partial c_{j}}{\partial t} \Gamma_{i j}^{k} e_{k} \\
& =\frac{\partial}{\partial t} \frac{\partial c}{\partial s}+\sum_{i j k} \frac{\partial c_{i}}{\partial s} \frac{\partial c_{j}}{\partial t} \Gamma_{i j}^{k} e_{k} \\
& =D_{t} \frac{\partial c}{\partial s}
\end{aligned}
$$

(2) Recall a curve is geodesic if $\left\|\frac{d}{d t} \gamma(t)\right\|_{\gamma(t)}$ is constant and $\left.\frac{d}{d s}\right|_{s=0} \int_{a}^{b}\left\|\frac{d}{d t} \gamma(t, s)\right\|_{\gamma(t, s)} d t=0$ for any variation of $\gamma(t)$. We first prove that if $D_{t} \gamma^{\prime}=0$ then it is a geodesic. For the first criteria, we use (5) and get

$$
\frac{d}{d t}\left\|\gamma^{\prime}(t)\right\|_{\gamma(t)}^{2}=\left\langle D_{t} \gamma^{\prime}, \gamma^{\prime}\right\rangle_{\gamma(t)}+\left\langle\gamma^{\prime}, D_{t} \gamma^{\prime}\right\rangle_{\gamma(t)}=0
$$


Hence, $\left\|\frac{d}{d t} \gamma(t)\right\|_{\gamma(t)}$ is a constant. For the second criteria, we again use (5) and then (6) to get

$$
\begin{aligned}
& \frac{d}{d s} \int_{a}^{b}\left\|\frac{d}{d t} \gamma(t, s)\right\|_{\gamma(t, s)} d t \\
& \stackrel{\triangleq}{=} \int_{a}^{b} \frac{1}{\left\|\frac{d}{d t} \gamma(t, s)\right\|_{\gamma(t, s)}}\left\langle D_{s} \frac{d}{d t} \gamma(t, s), \frac{d}{d t} \gamma(t, s)\right\rangle_{\gamma(t, s)} d t \\
& \stackrel{\boldsymbol{中}}{=} \int_{a}^{b} \frac{1}{\left\|\frac{d}{d t} \gamma(t, s)\right\|_{\gamma(t, s)}}\left\langle D_{t} \frac{d}{d s} \gamma(t, s), \frac{d}{d t} \gamma(t, s)\right\rangle_{\gamma(t, s)} d t \\
& \stackrel{\boldsymbol{\oplus}}{=} \frac{1}{\left\|\frac{d}{d t} \gamma(t, s)\right\|_{\gamma(t, s)}} \int_{a}^{b} \frac{d}{d t}\left\langle\frac{d}{d s} \gamma(t, s), \frac{d}{d t} \gamma(t, s)\right\rangle_{\gamma(t, s)}-\left\langle\frac{d}{d s} \gamma(t, s), D_{t} \frac{d}{d t} \gamma(t, s)\right\rangle_{\gamma(t, s)} d t \\
&= \frac{1}{\left\|\frac{d}{d t} \gamma(t, s)\right\|_{\gamma(t, s)}}\left(\left\langle\frac{d}{d s} \gamma(b, s), \frac{d}{d t} \gamma(b, s)\right\rangle_{\gamma(b, s)}-\left\langle\frac{d}{d s} \gamma(a, s), \frac{d}{d t} \gamma(a, s)\right\rangle_{\gamma(b, s)}\right)
\end{aligned}
$$

where we used (5) on $\diamond$, (6) on $\boldsymbol{\phi}$ and used (5) and $\left\|\frac{d}{d t} \gamma(t, s)\right\|_{\gamma(t, s)}$ is a constant with respect to $t$ on $\boldsymbol{\phi}$. In the last line, we use the fact that $D_{t} \frac{d}{d t} \gamma(t, s)=0$. Since $\frac{d}{d s} \gamma(a, s)=\frac{d}{d s} \gamma(b, s)=0$, we have that $\frac{d}{d s} \int_{a}^{b}\left\|\frac{d}{d t} \gamma(t, s)\right\|_{\gamma(t, s)} d t=0$.

To prove that any geodesic satisfies $D_{t} \gamma^{\prime}=0$, by the calculation in (A.4), we have that

$$
0=\int_{a}^{b}\left\langle\frac{d}{d s} \gamma(t, s), D_{t} \frac{d}{d t} \gamma(t, s)\right\rangle_{\gamma(t, s)} d t
$$

for any variation $\gamma(t, s)$ of $\gamma(t)$. Since $\frac{d}{d s} \gamma(t, s)$ is chosen by us, we can put $\frac{d}{d s} \gamma(t, s)=D_{t} \frac{d}{d t} \gamma(t, s)$ and this gives that $\int_{a}^{b}\left\|D_{t} \frac{d}{d t} \gamma(t, s)\right\|_{2}^{2} d t=0$. Hence, we have the result.

Exercise 93. Prove the first part of Theorem 6.

Proof. Note that

$$
\begin{aligned}
D_{t} D_{t} u & =\nabla_{c^{\prime}} \nabla_{c^{\prime}} \frac{\partial c}{\partial s}=\nabla_{c^{\prime}} \nabla_{\frac{\partial c}{\partial s}} \frac{\partial c}{\partial t} \\
& =\nabla_{\frac{\partial c}{\partial s}} \nabla_{c^{\prime}} \frac{\partial c}{\partial t}-R\left(\frac{\partial c}{\partial s}, \frac{\partial c}{\partial t}\right) \frac{\partial c}{\partial t} \\
& =-R\left(\frac{\partial c}{\partial s}, \frac{\partial c}{\partial t}\right) \frac{\partial c}{\partial t}
\end{aligned}
$$

where we used Fact 3 in the first equality, Fact 4 in the second equality and $\nabla_{c^{\prime}} \frac{\partial c}{\partial t}=0$ in the last equality. Once we fix $s=0$, the partial derivatives become derivatives in $t$. 